\title{
Resonant and magnetic X-ray diffraction by polarized synchrotron radiation
}

\author{
Luigi Paolasini ${ }^{\mathrm{a}}$ \\ European Synchrotron Radiation Facility, 6, Rue Jules Horowitz, BP. 220, 38043 Grenoble Cedex, \\ France
}

\begin{abstract}
The aim of these notes is to introduce the experimental methods and theoretical works in the domain of magnetic and resonant $\mathrm{x}$-rays scattering on single crystals to investigate the electronic and magnetic long range order in strongly correlated electron systems. We highlight the role of the x-rays polarization analysis and control to determine the magnetic structure of materials and, more generally, the electronic long range order parameters responsible for the phase transitions.
\end{abstract}

\section{INTRODUCTION}

The investigation of the electronic and magnetic order parameters and their evolution as a function of thermodynamic variables is one of the main research fields in the domain of strongly correlated electron systems and complex materials, in which different and often competing order parameters are present, giving rise to many spectacular manifestations of quantum physics in condensed matter. In fact, it is well known that the complex interaction between charge, orbital and spin degrees of freedom can determine peculiar phenomena like superconductivity, colossal magnetoresistance, metal-insulator transitions, charge ordering. The explanation and the description of these systems is non trivial, and they are a challenge for theoretical models that aim to calculate the electronic structure as it is necessary to correctly treat electron-electron interactions.

Several scattering techniques using neutrons, $\mathrm{x}$-rays, muons, electrons are exploited to investigate the microscopic electronic correlations in solids. Among these different techniques, neutrons have occupied an important role in the studies of magnetism in condensed matter, and they represent one of the most important tools to investigate the static and dynamic magnetic correlations in crystalline materials. The advent of modern synchrotron radiation sources has opened new possibilities to exploit hard x-rays as a probe to study magnetic systems. In the last fifteen years the number of beamlines dedicated to magnetic investigations has been growing at an amazing rate, and today every major synchrotron light source has a large variety of specialized instruments.

The peculiar characteristics of synchrotron radiation, such as the capability to probe small scattering volumes (small crystals or magnetic thin films) with a highly collimated and intense beam, the high

\footnotetext{
ae-mail: paolasini@esrf.eu
}

This is an Open Access article distributed under the terms of the Creative Commons Attribution License 4.0, which permits unrestricted use, distribution, and reproduction in any medium, provided the original work is properly cited. 


\section{Collection SFN}

degree of photon beam polarization and the energy tunability, put synchrotron radiation studies at the forefront of the modern scattering techniques.

The aim of this work is to review the experimental and theoretical works in the domain of magnetic and resonant x-ray scattering (RXS) on single crystals to investigate the electronic and magnetic long range order in strongly correlated electron systems. We shows how it is possible to exploit the basic properties of synchrotron radiation interaction with the bound electrons in solid to determine the magnetic structure of materials and, more generally, the electronic long range order parameters responsible for the phase transitions. We will describe then some experiments addressed to the understanding of selected problems in condensed matter physics, with particular emphasis to those in which synchrotron radiation methods have been proven to be particularly suited with respect to other complementary techniques. Most of these studies have promoted a revisitation of x-ray scattering theories, which, in turn, have suggested new experimental challenges, improving the methods and the instrumentation in order to perform the new demanding measurements.

\section{ELEMENTS OF THEORY OF MAGNETIC AND RESONANT X-RAY DIFFRACTION}

The aim of any spectroscopic experiment is to determine the temporal evolution of the physical state of the system constituted by a large ensemble of particles at the thermal equilibrium at a given temperature. For this purpose, we measure the variations of the dynamical variables of the probe after the interaction with the sample by the scattering process (elastic and inelastic). Neutrons or hard X-rays scattering have energies comparable with the sample eigenstates, and the quantity which is directly measured in a scattering experiment is the differential cross section, defined as the probability for a particle of incident energy $E_{i}$ to be scattered along a given direction, into an unitary solid angle $\Delta \Omega$, with a final energy $E_{f}$.

The fundamental interaction of x-rays with bound electrons can be due to the electron charge or by the coupling between the electric and magnetic field of the incident photons with the atomic magnetic moment. For energies $\hbar \omega<100 \mathrm{keV}$ the $\mathrm{x}$-rays interact weakly with bulk samples, and the scattering maybe accurately treated in the lowest Born-Oppenheimer approximation. Photons are defined by the initial state $|\mathbf{k}, \hat{\epsilon}\rangle$ characterized by wavevector $\mathbf{k}$, polarization $\hat{\epsilon}$ and energy $\hbar \omega_{k}$. Initially the electrons are found in the ground state $|g\rangle$, an eigenstate of the unperturbed electron Hamiltonian $\mathcal{H}_{e l}$ with energy $E_{g}$. The interaction between electrons and photons creates a new final eigenstate $|f\rangle$ for the electrons with an energy $E_{f}$ and a scattered photon in the state $\left|\mathbf{k}^{\prime}, \hat{\epsilon}^{\prime}\right\rangle$ with an energy $\hbar \omega_{k^{\prime}}$. Here we consider the differential cross section for the coherent elastic x-ray scattering on single crystals $\left(\hbar \omega_{k}=\hbar \omega_{k^{\prime}}\right.$ and $E_{g}=E_{f}$ ), in which the individual atomic scattering amplitudes interfere at different lattice sites $n$ :

$$
\frac{d \sigma}{d \Omega}=r_{0}^{2}\left|\sum_{n} e^{i \mathbf{Q} \cdot \mathbf{R}_{n}} f_{n}\left(\mathbf{k}, \mathbf{k}^{\prime}, \hat{\epsilon}, \hat{\epsilon}^{\prime}, \hbar \omega_{k}\right)\right|^{2}
$$

where and $r_{0}=e^{2} / m c^{2} \approx 2.82 \cdot 10^{-5} \AA$ is the classical electron radius, $f_{n}\left(\mathbf{k}, \mathbf{k}^{\prime}, \hat{\epsilon}, \hat{\epsilon}^{\prime}, \hbar \omega_{k}\right)$ is the scattering amplitude of the electrons at site $n, \mathbf{R}_{n}$ is the position of the $n^{\text {th }}$ site in the crystal, $\mathbf{Q}=\mathbf{k}-\mathbf{k}^{\prime}$ is the scattering vector.

In x-ray scattering experiments the scattering amplitude $f_{n}\left(\mathbf{k}, \mathbf{k}^{\prime}, \hat{\epsilon}, \hat{\epsilon}^{\prime}, \hbar \omega_{k}\right)=f_{n}^{0}+f_{n}^{\text {magn. }}+f_{n}^{\prime}+$ $i f_{n}^{\prime \prime}$ has two regimes, determined by the incident photon energy: the non-resonant limit contains the energy independent part of the scattering amplitude $f_{n}^{0}+f_{n}^{\text {magn. }}$, and the resonant x-ray scattering regime contains the real $f^{\prime}$ and imaginary $f^{\prime \prime}$ part of the energy-dependent terms. The non-resonant regime includes both the Thomson scattering amplitude $f^{0}$ and the magnetic scattering amplitude 
$f^{\text {magn. }}$. The resonant term $f^{R X S}=f^{\prime}+i f^{\prime \prime}$, also called anomalous or dispersive term, appears when the incident X-ray energy lies near an absorption edge (i.e. K, L, M ... absorption edges) as a consequence of the photo-absorption effect, and corresponds to transitions of core electrons into available electronic states above the Fermi energy level.

Following the perturbative method developed by Blume [9], which considers an unperturbed system described by the electron and non-interacting photon Hamiltonians, the scattering amplitudes $f\left(\mathbf{k}, \mathbf{k}^{\prime}, \hat{\epsilon}, \hat{\epsilon}^{\prime}, \hbar \omega_{k}\right)$ can be developed in non-resonant and resonant scattering amplitudes, by assuming that the unperturbed radiation is monochromatic, and the vector potential which characterizes the radiation field is a linearly polarized plane wave.

We report here the final expression of elastic scattering amplitudes, in which the non-resonant and the resonant regimes are calculated separately [10]:

$$
\begin{aligned}
f\left(\mathbf{k}, \mathbf{k}^{\prime}, \hat{\boldsymbol{\epsilon}}, \hat{\boldsymbol{\epsilon}}^{\prime}, \hbar \omega_{k}\right)= & -\left\langle g\left|\sum_{j} e^{i \mathbf{Q} \cdot \mathbf{r}_{j}}\right| g\right\rangle \hat{\boldsymbol{\epsilon}}^{\prime *} \cdot \hat{\boldsymbol{\epsilon}}-i \frac{\hbar \omega_{k}}{m c^{2}} \hat{\boldsymbol{\epsilon}}^{\prime *} \\
& \cdot\left\langle g\left|\sum_{j} e^{i \mathbf{Q} \cdot \mathbf{r}_{\mathbf{j}}}\left(\frac{i \mathbf{Q} \times \mathbf{p}_{j}}{\hbar Q^{2}} \cdot \mathbf{A}-\mathbf{s}_{j} \cdot \mathbf{B}\right)\right| g\right\rangle \cdot \hat{\boldsymbol{\epsilon}} \\
& +\frac{1}{m} \sum_{c} \frac{E_{g}-E_{c}}{\hbar \omega_{k}}\left(\frac{\hat{\boldsymbol{\epsilon}}^{\prime *} \cdot\left\langle g\left|\tilde{O}^{\dagger}\left(\mathbf{k}^{\prime}\right)\right| c\right\rangle\langle c|\tilde{O}(\mathbf{k})| g\rangle \cdot \hat{\boldsymbol{\epsilon}}}{E_{g}-E_{c}+\hbar \omega_{k}-i \Gamma_{c} / 2}\right. \\
& \left.-\frac{\hat{\boldsymbol{\epsilon}} \cdot\langle g|\tilde{O}(\mathbf{k})| c\rangle\left\langle c\left|\tilde{O}^{\dagger}\left(\mathbf{k}^{\prime}\right)\right| g\right\rangle \cdot \hat{\boldsymbol{\epsilon}}^{* *}}{E_{g}-E_{c}-\hbar \omega_{k}}\right)
\end{aligned}
$$

where the first term is the Thomson contribution $f^{0}$ associated to the isotropic charge density distribution, the second term describes the non-resonant X-ray magnetic scattering (NRXMS) amplitude $f^{m a g n}$, and it is associated to the orbital and spin magnetic density distribution. The vectors $\mathbf{A}$ and $\mathbf{B}$ contain the coupling between the photon polarisation and the scattering wave vectors for the orbital and the spin part of the NRXS amplitude, respectively. The last two terms are the resonant contribution $f^{R X S}=f^{\prime}+i f^{\prime \prime}$, associated to the core-hole resonant transitions in which the initial photon has been annihilated first (third term) or the final photon has first been created (fourth term) and a photoelectron is created in the $|c\rangle$ with energy $E_{c}$ and lifetime [12]. The current operator $\tilde{O}(\mathbf{k})=\sum_{j} e^{i \mathbf{k} \cdot \mathbf{r}_{j}}\left(\mathbf{p}_{j}-i \hbar \mathbf{k} \times \mathbf{s}_{j}\right)$ probes the intermediate states $|c\rangle$ when the incident energy is tuned across a characterisc energy $\hbar \omega_{k}=E_{g}-E_{c}$, and were $\Gamma_{c}$ is related to the life time $2 \pi \hbar / \Gamma_{c}$ of the excited level $|c\rangle$ with energy $E_{c}$.

The sums in Eq. (2.2) are extended to those electrons which interact with the radiation field, namely all the core and valence electrons $Z$ for the Thomson term $\left(f^{0} \propto Z r_{0}\right)$, but only the electrons occupying the partially-filled shells in the case of the magnetic term $\left(f^{\text {magn. }} \propto Z^{\text {magn }} \cdot r_{0}\right)$, and the resonant electrons $\left(f^{R X S} \propto Z^{\text {res. }} r_{0}\right)$ for resonant terms. Notice that we have omitted the thermal population factor because we assume for simplicity that only one state is populated at $\mathrm{T}=0$.

\subsection{Non-resonant X-rays scattering}

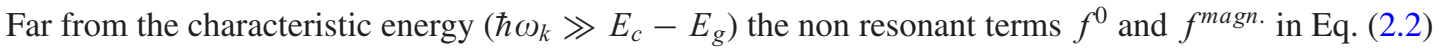
are dominant. A subject of debate is the determination of the limit of this assumption, which is certainly valid when the incident photon energy is tuned above the absorption edges (and considering the higher limit $\left.\hbar \omega_{k} \ll m c^{2}\right)$. However, for many experimental reasons, most of the NRXMS experiments are performed below the absorption edges, in which the presence of the resonant contribution could affect 


\section{Collection SFN}

the measured scattered intensities. In these cases a necessary and careful estimation of the resonant contamination must be evaluated case by case ${ }^{1}$.

The Thomson scattering amplitude is directly related to the Fourier transform of the electron charge density. The NRXMS amplitude is very weak when compared to the Thomson term, because the relativistic character of the electron-photon interaction introduces a scale factor $(\hbar Q) /(2 \pi m c)=$ $2\left(\lambda_{c} / \lambda\right) \sin \theta$ between magnetic scattering and Thomson scattering amplitudes, respectively, where here $\lambda_{c}$ is the Compton wavelength of the electron $\left(\lambda_{c}=2 \hbar / m c=0.002426 \mathrm{~nm}\right)$. Compared to the classical charge scattering the pure magnetic scattering amplitude is thus significantly reduced by a factor of $\hbar \omega_{k} / m c^{2}$, and the ratio between the magnetic and charge scattering cross sections can be estimated as:

$$
\frac{\sigma_{\text {mag }}}{\sigma_{\text {charge }}} \simeq\left(\frac{\hbar \omega_{k}}{m c^{2}}\right)^{2}\left(\frac{Z^{\text {magn. }}}{Z}\right)^{2}\langle M\rangle^{2}\left(\frac{f_{m}}{f}\right)^{2}
$$

where $m c^{2}=511 \mathrm{keV}$ is the rest mass of an electron, $Z^{m a g n}$. is the number of magnetic electrons per atom, $Z$ the number of electrons per atom and $f_{m}$ and $f$ are the magnetic and charge form factors, respectively. $\langle M\rangle$ is the magnetic order parameter, being equal to one only at low temperature, when the system is magnetically saturated. For example in the case of $\mathrm{Mn}^{2+}$ ion this ratio becomes $\frac{\sigma_{\text {mag }}}{\sigma_{\text {charge }}} \sim 10^{-6}$ (assuming that $f_{m} / f \sim 1$ ).

The NRXMS amplitude $f^{\text {magn. }}$ could be expanded in terms of the Fourier transforms of orbital and spin magnetization density $\mathbf{L}(\mathbf{Q})=\left\langle g\left|\sum_{j} \mathbf{l}_{j} e^{i \mathbf{Q} \cdot \mathbf{r}_{j}}\right| g\right\rangle$ and $\mathbf{S}(\mathbf{Q})=\left\langle g\left|\sum_{j} \mathbf{S}_{j} e^{i \mathbf{Q} \cdot \mathbf{r}_{j}}\right| g\right\rangle$, respectively, directly related to the magnetic form factors. Then the $f^{\text {magn }}$ amplitude assumes the operative form:

$$
f^{m a g n} \cdot(\mathbf{Q})=-i \frac{\hbar \omega_{k}}{m c^{2}}\left(\mathbf{L}(\mathbf{Q}) \cdot \mathbf{P}_{L}+\mathbf{S}(\mathbf{Q}) \cdot \mathbf{P}_{S}\right)
$$

where $\mathbf{P}_{L}$ and $\mathbf{P}_{S}$ are vectors describing the polarization and wavevector dependence of the magnetic scattering process as:

$$
\begin{aligned}
& \mathbf{P}_{L}=-2 \sin ^{2} \theta\left[\mathbf{Q} \times\left[\left(\hat{\epsilon}^{*} \times \hat{\epsilon}\right) \times \mathbf{Q}\right]\right] \\
& \mathbf{P}_{S}=\hat{\boldsymbol{\epsilon}}^{* *} \times \hat{\epsilon}+\left(\hat{\mathbf{k}}^{\prime} \times \hat{\boldsymbol{\epsilon}}^{*}\right)\left(\hat{\mathbf{k}}^{\prime} \cdot \hat{\boldsymbol{\epsilon}}\right)-(\hat{\mathbf{k}} \times \hat{\boldsymbol{\epsilon}})\left(\hat{\mathbf{k}} \cdot \hat{\boldsymbol{\epsilon}}^{\prime *}\right)-\left(\hat{\mathbf{k}}^{\prime} \times \hat{\boldsymbol{\epsilon}}^{\prime *}\right) \times(\hat{\mathbf{k}} \times \hat{\boldsymbol{\epsilon}}) .
\end{aligned}
$$

These expressions could be simplified by choosing an appropriate coordinate reference system for the polarization vectors with respect to the scattering geometry, as we will see in Section 3.2.1 [11]. It can be seen also that, by taking advantage of the geometrical prefactors for spin and orbital moments, x-ray magnetic scattering provides the possibility to separate the spin $\mathbf{S}$ and orbital $\mathbf{L}$ moment contributions in the total magnetization density.

\subsection{Resonant X-rays scattering}

In diffraction or in absorption techniques an incoming photon is absorbed and a core electron is promoted to an empty continuum state or an empty bound state, depending on the photon energy. For example, when an electron is promoted to a continuum state well above the absorption edge (more than $30 \mathrm{eV}$ ), we have the Extended X-ray Absorption Fine Structure (EXAFS) regime in the case of the absorption or the Extended Diffraction Anomalous Fine Structure (EDAFS) in the case of the diffraction. For energies less the $30 \mathrm{eV}$ from the absorption edge, we have the X-rays Absorption Near Edge Structure (XANES) regime for the absorption and Diffraction Anomalous Near Edge Structure (DANES) for the diffraction. With RXS here we include the general theory of photon-electron

1 The determination of the limit of this assumption can be found in the Supporting Online Material of H. Walker et al., Science 333, 1273 (2011). 
interaction in the non-relativistic limit (when NRXMS terms are neglected) which can be applied to both absorption and diffraction regimes, because the scattered intensities are related to the matrix elements of the interacting operator between the ground state wave function and the excited one.

The link between RXS diffraction and absorption spectroscopies is given in the forward direction by the imaginary part of scattering amplitude $f^{\prime \prime}(\mathbf{Q}=\mathbf{0})$, which is related to the absorption cross section:

$$
\sigma=\frac{4 \pi}{|\mathbf{k}|} \operatorname{Im}\left[\hat{\epsilon}^{*} \cdot f^{\prime \prime}(\mathbf{Q}=0)\right]
$$

The peculiarity of RXS diffraction with respect to the other absorption spectroscopies is that the different scattering amplitudes interfere coherently, and this allows the sensitivity to long range order and, in favorable cases, to single out the individual high-order multipoles with their symmetries.

Notice that the wavevector dependence of the RXS amplitude $f^{R X S}=f^{\prime}+i f^{\prime \prime}$ is often very small and in most of the cases could be considered energy dependent. In fact these terms arise mostly from inner-shell electrons, and thus are less sensitive to the scattering angles. Usually, in the analysis of the RXS diffraction data, it may be quite accurate to use zero-angle value for $f^{\prime}$ and $f^{\prime \prime}$ which are derived from absorption data or calculated from theory in the dipole approximation, where the atomic size effects are neglected.

Different approaches can be used to expand the RXS amplitude and to evaluate the matrix elements which contain the current operators $\tilde{O}(\mathbf{k})$ and $\tilde{O}^{\dagger}\left(\mathbf{k}^{\prime}\right)$ in Eq. (2.2). Following the Hannon model for resonant exchange scattering [13], two possible photoelectron transition are considered in RXS regime: the electric dipole (E1) and the electric quadrupole (E2) transitions, which are related to the symmetry of the electronic shell and to the type of orbitals probed in the intermediate states. Practical formulas for the magnetic part of RXS can be found in Ref. [14], in which the Hannon's terms are developed in a cartesian reference system. However these expressions are only valid for SU(2) symmetries, i.e., spherical symmetry broken by an axial vector (for instance, the magnetic moment for the magnetic terms).

Table 1 shows the absorption edges exploited for RXS studies and associated to the elements of interest for resonant magnetic diffraction. The lines in italic represent absorption edges in the soft x-ray regime, where the scattering conditions are not fulfilled for most of the ordered magnetic structures, but with a huge enhancement of the resonant magnetic amplitude, as shown in the last column (L-edges of transition metals and M-edges of rare earths). The most important edges for hard RXS cover the energy range 3.5-15 keV, which includes K-edges of transition metals, the L-edges of Rare-Earths and both the L-edges and the M-edges of actinides. The last column of Table 1 shows the estimation of the magnetic scattering amplitude for the different shells. Notice the strong enhancement at M-edges of rare earths and actinides, arising from the asymmetry of the transition probabilities coupled with the spin-orbit splitting either in the ground state or in the intermediate states. The sensitivity to magnetic moments comes from the Pauli principle and the spin-orbit interaction which ensures the availability of the intermediate states near the resonance energy. The spin-orbit interaction plays a role both in the core levels with nonvanishing orbital moment $l \neq 0$, as in the case of $\mathrm{L}_{2}$ and $\mathrm{L}_{3}$ edges of rare earths, or in the much weaker spin-orbit interaction of valence states, as in the case of K-edge of transition metals, where the $1 s$ core levels have a vanishing spin-orbit interaction $l=0$, as shown in Table 1 . The extension of Hannon formula is necessary for non-centosymmetric systems, as we will see for example in Section 4.1 for the case of forbidden lattice reflections at the K-edge resonance in $\mathrm{V}_{2} \mathrm{O}_{3}$ [15].

Here we summarise the main results of the multipole expansion development of RXS terms by restricting the analysis to the relevant multipole contributions detected by RXS, i.e electric dipole E1 and electric quadrupole E2 transitions, by omitting the spin and orbital parts in the dispersive term (M1 and M2 matrix elements), which are included in the general expansion of the current operator in the so called $\vec{r}$-form (see for more details Ref. [18]). 


\section{Collection SFN}

Table 1. Photoabsorption edges for different series of magnetic elements, with the characteristic energy ranges $(\mathrm{keV})$, wavelength $\lambda(\AA)$ and the allowed atomic transitions to the electronic shells. The rightmost column indicates the extimated resonant magnetic scattering amplitudes expressed in $\mathrm{r}_{0}$ units [32].

\begin{tabular}{llccccc}
\hline Series & $\begin{array}{c}\text { Abs. } \\
\text { edge }\end{array}$ & $\begin{array}{c}\text { Energy } \\
(\mathrm{keV})\end{array}$ & $\begin{array}{c}\lambda \\
(\AA)\end{array}$ & Shells & Type & $\begin{array}{c}\text { RMXS } \\
\left(\mathrm{r}_{0}\right)\end{array}$ \\
\hline \hline 3d & $L_{2,3}$ & $0.4-1.0$ & $12-30$ & $2 p \rightarrow 3 d$ & $E 1$ & $\sim 100$ \\
& $\mathbf{K}$ & $4.5-9.5$ & $1.3-2.7$ & $\begin{array}{c}1 \mathrm{~s} \rightarrow 4 \mathrm{p} \\
1 \mathrm{~s} \rightarrow 3 \mathrm{~d}\end{array}$ & $\begin{array}{c}\mathrm{E} 1 \\
\mathrm{E} 2\end{array}$ & $\sim 0.02$ \\
& & & & 0.01 \\
\hline $\mathbf{5 d}$ & $\mathbf{L}_{2,3}$ & $5.4-14$ & $0.9-2.2$ & $2 p \rightarrow 5 d$ & $\mathrm{E} 1$ & $\sim 1-10$ \\
\hline $\mathbf{4 f}$ & $\mathbf{L}_{2,3}$ & $5.7-10.3$ & $1.2-2.2$ & $2 \mathrm{p} \rightarrow 5 \mathrm{~d}$ & $\mathrm{E} 1$ & $\sim 0.10$ \\
& & & & $2 \mathrm{p} \rightarrow 4 \mathrm{f}$ & $\mathrm{E} 2$ & $\sim 0.05$ \\
& $M_{4,5}$ & $0.9-1.6$ & $7.7-13.8$ & $2 d \rightarrow 4 f$ & $E 1$ & $\sim 100-200$ \\
\hline $\mathbf{5 f}$ & $\mathbf{L}_{2,3}$ & $17-21$ & $0.6-0.7$ & $2 \mathrm{p} \rightarrow 6 \mathrm{~d}$ & $\mathrm{E} 1$ & $\sim 0.05$ \\
& & & & $2 \mathrm{p} \rightarrow 4 \mathrm{f}$ & $\mathrm{E} 2$ & $\sim 0.01$ \\
& $\mathbf{M}_{4,5}$ & $3.5-4.5$ & $2.7-6$ & $3 \mathrm{~d} \rightarrow 5 \mathrm{f}$ & $\mathrm{E} 1$ & $\sim 10$ \\
\hline & & & & & &
\end{tabular}

Starting from second order terms in Eq. (2.2) containing the resonant contribution, and after some approximations, the resonant scattering amplitude becomes:

$$
f^{R X S} \approx-\frac{1}{m} \sum_{c} \frac{E_{g}-E_{c}}{\hbar \omega_{k}} \cdot \frac{\left\langle g\left|\sum_{j} e^{-i \mathbf{k}^{\prime} \cdot \mathbf{r}_{j}} \hat{\boldsymbol{\epsilon}}^{\prime *} \cdot \mathbf{p}_{j}\right| c\right\rangle\left\langle c\left|\sum_{j} e^{i \mathbf{k} \cdot \mathbf{r}_{j}} \hat{\boldsymbol{\epsilon}} \cdot \mathbf{p}_{j}\right| g\right\rangle}{E_{g}-E_{c}+\hbar \omega_{k}-i \Gamma_{c} / 2} .
$$

The Eq. (2.7) was developed by Hannon et al. [13] within the dipole approximation, i.e. by developing $e^{i \mathbf{k} \cdot \mathbf{r}_{j}} \approx 1+i \mathbf{k} \cdot \mathbf{r}_{j}-\left(\mathbf{k} \cdot \mathbf{r}_{j}\right)^{2} / 2+\ldots$ as a power series and retaining only the two first dominant contributions. This approximation is valid as far as $\mathrm{kr} \ll 1$, and in the general case the multipole expansion on a plane wave is based on the expansion in spherical harmonics (Bessel functions for the radial part and Legendre polynomials for the angular part).

The Hannon's term described by the Eq. (2.7) can be rewritten in three distinct terms by using the previous relations which include the dipole $\sum_{j} \mathbf{r}_{j}$ and the quadrupole $\sum_{j} \frac{i}{2} \mathbf{r}_{j}\left(\mathbf{k} \cdot \mathbf{r}_{j}\right)$ operators:

$$
\begin{aligned}
f^{R X S} \approx & m \sum_{c} \frac{\left(E_{c}-E_{g}\right)^{3}}{\hbar^{3} \omega_{k}\left(E_{g}-E_{c}+\hbar \omega_{k}-i \Gamma_{c} / 2\right)}\left[\hat{\boldsymbol{\epsilon}}^{\prime *} \cdot\left\langle g\left|\sum_{j} \mathbf{r}_{j}\right| c\right\rangle\left\langle c\left|\sum_{i} \mathbf{r}_{i}\right| g\right\rangle \cdot \hat{\boldsymbol{\epsilon}}\right. \\
& -i \hat{\boldsymbol{\epsilon}}^{\prime *} \cdot\left\langle g\left|\sum_{j} \frac{1}{2} \mathbf{r}_{j}\left(\mathbf{k}^{\prime} \cdot \mathbf{r}_{j}\right)\right| c\right\rangle\left\langle c\left|\sum_{i} \mathbf{r}_{i}\right| g\right\rangle \cdot \hat{\boldsymbol{\epsilon}}+i \hat{\boldsymbol{\epsilon}}^{*} \cdot\left\langle g\left|\sum_{j} \mathbf{r}_{j}\right| c\right\rangle\left\langle c\left|\sum_{i} \frac{1}{2} \mathbf{r}_{i}\left(\mathbf{k} \cdot \mathbf{r}_{i}\right)\right| g\right\rangle \cdot \hat{\boldsymbol{\epsilon}} \\
& \left.+\hat{\boldsymbol{\epsilon}}^{\prime *} \cdot\left\langle g\left|\sum_{j} \frac{1}{2} \mathbf{r}_{j}\left(\mathbf{k}^{\prime} \cdot \mathbf{r}_{j}\right)\right| c\right\rangle\left\langle c\left|\sum_{i} \frac{1}{2} \mathbf{r}_{i}\left(\mathbf{k} \cdot \mathbf{r}_{i}\right)\right| g\right\rangle \cdot \hat{\boldsymbol{\epsilon}}\right]
\end{aligned}
$$

where the first term contains the dipole-dipole operators (E1-E1), the second and the third terms the dipole-quadrupole operators (E1-E2) and the fourth term the quadrupole-quadrupole operator (E2-E2). Using a cartesian reference coordinate system for the photon polarization, the Eq. (2.8) can be written in the form:

$$
\begin{aligned}
f^{R X S} \approx & m \sum_{c} \frac{\left(E_{c}-E_{g}\right)^{3}}{\hbar^{3} \omega_{k}\left(E_{g}-E_{c}+\hbar \omega_{k}-i \Gamma_{c} / 2\right)}\left[\sum_{\alpha, \beta} \epsilon_{\alpha}^{\prime *} \epsilon_{\beta} D_{\alpha \beta}+\frac{i}{2} \sum_{\alpha, \beta, \gamma} \epsilon_{\alpha}^{\prime *} \epsilon_{\beta}\left(k_{\gamma} I_{\alpha \beta \gamma}-k_{\gamma}^{\prime} I_{\alpha \beta \gamma}^{*}\right)\right. \\
& \left.+\frac{1}{4} \sum_{\alpha, \beta, \gamma, \delta} \epsilon_{\alpha}^{*} \epsilon_{\beta} k_{\gamma}^{\prime} k_{\delta} Q_{\alpha \beta \gamma \delta}\right]
\end{aligned}
$$


where $\alpha, \beta, \gamma, \delta$ are indexes which vary independently over the three cartesian directions $x, y$, $z$, and we have used the following shorthand notation for the transition matrix elements $D_{\alpha \beta}=$ $\left\langle g\left|\sum_{j} r_{j}^{\alpha}\right| c\right\rangle\left\langle c\left|\sum_{i} r_{i}^{\beta}\right| g\right\rangle, I_{\alpha \beta \gamma}=\left\langle g\left|\sum_{j} r_{j}^{\alpha}\right| c\right\rangle\left\langle c\left|\sum_{i} r_{i}^{\beta} r_{i}^{\gamma}\right| g\right\rangle$ and $Q_{\alpha \beta \gamma \delta}=\left\langle g\left|\sum_{j} r_{j}^{\alpha} r_{j}^{\delta}\right| c\right\rangle\left\langle c\left|\sum_{i} r_{i}^{\beta} r_{i}^{\gamma}\right| g\right\rangle$. These transition matrix elements $D_{\alpha \beta}, I_{\alpha \beta \gamma}$ and $Q_{\alpha \beta \gamma \delta}$, are associated to (E1-E1), (E1-E2) and (E2-E2) contributions and are characterized by cartesian tensors of second, third and fourth rank, respectively.

The magnetic atoms in the unit cell are related by symmetry operations and the site symmetry of the individual atoms has important implication on the possible allowed resonant transitions defined by the matrix elements of Eq. (2.9). It is therefore important to determine the invariant behaviour of these tensors $D, I$ and $Q$ under the following symmetry transformations of the coordinate system: the space inversion or parity which corresponds to the under inversion of space coordinates, i.e. $(x, y, z) \rightarrow(-x,-y,-z)$, and the time reversal operation change of the direction of magnetic moments, or, equivalently, change $\mathbf{k} \rightarrow-\mathbf{k}$. Both tensors $D$ and $Q$ are even under the space inversion symmetry. In fact they can be expressed as a product of two matrix elements both containing an odd or even number of coordinates $r_{i}$. This leads to an even final product. This is not true in the case of the tensor $I$ which is odd under inversion symmetry operation, as it is made of an odd number of position operators. As a result, if an atom sits in an inversion center, the $I$ tensor must be zero, or, in other words, dipolequadrupole transition (E1-E2) are only allowed for atoms breaking the inversion symmetry.

Each tensor element $D_{\alpha \beta}$ (or $I_{\alpha \beta \gamma}$, or $Q_{\alpha \beta \gamma \delta}$ ) can be decomposed in its irreducible components $T_{m}^{(p)}$ by making an appropriate basis change in the tensor space, and by considering the rotational symmetry operations [19]. For example, for the (E1-E1) dipole terms, the scattering amplitude can be written as:

$$
f^{R X S}(E 1-E 1) \propto \sum_{\alpha \beta} \epsilon_{\alpha}^{\prime} \epsilon_{\beta} D_{\alpha \beta}=\sum_{p=0,1,2} \sum_{m=-p}^{p}(-1)^{p+m} X_{-m}^{(p)} T_{m}^{(p)}
$$

where $X^{(p)}$ are spherical tensors constructed from the polarization vectors $\hat{\epsilon}$ and $\hat{\epsilon}^{\prime}$ starting from the cartesian tensors $\epsilon_{\alpha}$ and $\epsilon_{\beta}$, and developed in spherical tensors [20]. Specific expression of these spherical tensors can be found in Refs. [17, 21, 22].

In general the RXS amplitude is expressed as a scalar product of two irreducible spherical tensors:

$$
f_{j}^{R X S}=\sum_{p, m}(-1)^{p+m} X_{-m}^{(p)} F_{m}^{(p)}(j ; \omega)
$$

where $X_{-m}^{(p)}$ depends only on the incident and scattered polarization and wavevector, while $F_{m}^{(p)}(j ; \omega)$ is associated to the tensorial properties of the $\mathrm{j}$-atom, and can be represented in terms of multipole expansion. The rank $p$ defines the order of the multipole in the electromagnetic field expansion and the projection can take $(2 p+1)$ values that satisfy $(-p \leq m \leq p) . p=$ even tensors are time-even, i.e. invariant under time reversal, whereas $p=$ odd tensor are time-odd.

The classification of multipole transitions has been developed in Refs. [18, 21, 23-25], and a classification of the tensors associated to the multipole expansion are summarized in Table 2. For the tensor ranks $p=0,1,2$, (E1-E1) and (E2-E2) tensors represent the same physical quantities, though referred to states with different angular momentum. The classification of multipoles illustrated in Table 2 is dictated by the unique properties under time-reversal $\tilde{T}$ and parity $\tilde{P}$ transformations of $F^{(p)}$ tensors. For any given tensor rank $p=1,2,3,4$, there is just one electromagnetic multipole of the same rank $(1 \rightarrow$ dipole, $2 \rightarrow$ quadrupole, $3 \rightarrow$ octupole, $4 \rightarrow$ hexadecapole) with the same $\tilde{T}$ and $\tilde{P}$ properties. Notice that $\tilde{P}$-odd (E1-E2) tensors have both $\tilde{T}$-odd (sign - in the exponent) and $\tilde{T}$-even (sign + ) terms for any $p$, while $\tilde{P}$-even tensors (both (E1-E1) and (E2-E2)) are $\tilde{T}$-odd if of odd rank and $\tilde{T}$ even if of even rank. $\tilde{T}$-even tensors are associated to a charge multipoles, whereas $\tilde{T}$-odd tensors are magnetic.

As each $F^{(p)}$ is scalarly related to a corresponding irreducible tensor $X^{(p)}$ (as shown in Eq. (2.11)), they have the same $\tilde{T}$ and $\tilde{P}$ properties. For example, in both the (E1-E1) or (E2-E2) channels, 


\section{Collection SFN}

Table 2. Properties of multiple expansion tensors under time-reversal $\tilde{T}$ and parity $\tilde{P}$ operators (from Ref. [21]).

\begin{tabular}{|l|c|c|c|c|c|}
\hline Tensor & rank & $\tilde{T}$ & $\tilde{P}$ & Type & Multipole \\
\hline \hline$F^{(0)}(E 1-E 1)$ & 0 & + & + & charge & monopole \\
\hline$F^{(0)}(E 2-E 2)$ & 0 & + & + & charge & monopole \\
\hline$F^{(1)}(E 1-E 1)$ & 1 & - & + & magnetic & dipole \\
\hline$F^{(1)}(E 2-E 2)$ & 1 & - & + & magnetic & dipole \\
\hline$F^{(1+)}(E 1-E 2)$ & 1 & + & - & electric & dipole \\
\hline$F^{(1-)}(E 1-E 2)$ & 1 & - & - & polar toroidal & dipole \\
\hline$F^{(2)}(E 1-E 1)$ & 2 & + & + & electric & quadrupole \\
\hline$F^{(2)}(E 2-E 2)$ & 2 & + & + & electric & quadrupole \\
\hline$F^{(2+)}(E 1-E 2)$ & 2 & + & - & axial toroidal & quadrupole \\
\hline$F^{(2-)}(E 1-E 2)$ & 2 & - & - & magnetic & quadrupole \\
\hline$F^{(3)}(E 2-E 2)$ & 3 & - & + & magnetic & octupole \\
\hline$F^{(3+)}(E 1-E 2)$ & 3 & + & - & electric & octupole \\
\hline$F^{(3-)}(E 1-E 2)$ & 3 & - & - & polar toroidal & octupole \\
\hline$F^{(4)}(E 2-E 2)$ & 4 & + & + & electric & hexadecapole \\
\hline
\end{tabular}

$X^{(0)} \equiv \hat{\epsilon}^{* *} \cdot \hat{\epsilon}$ is a scalar (charge monopole, which corresponds to the Thomson term) and $X^{(1)} \equiv \hat{\epsilon}^{*} \times \hat{\epsilon}$ is a $\tilde{T}$-odd and $\tilde{P}$-even vector (the magnetic dipole term). Analogously, in the (E1-E2) channel, the previous quantities combine with the two $\tilde{P}$-odd vectors $\left(\mathbf{k}+\mathbf{k}^{\prime}\right)$, which is $\tilde{T}$-odd, and $\left(\mathbf{k}-\mathbf{k}^{\prime}\right)$, which is $\tilde{T}$-even.

The tensorial nature of resonant $\mathrm{x}$-ray scattering amplitudes has important consequences in the X-ray scattering cross section Eq. (2.1), because forbidden lattice reflections appear at resonance, as demonstrated in seminal experimental works of Templeton [26], with timely analysis of Dimitrienko [27], and the observation of Finkelstein [28], with the theoretical interpretation of Carra [29].

RXS is a unique tool to investigate the physics of multipolar ordered parameters and is one of the most exciting research domain of investigation of strongly correlated electron systems. However, it must be remarked that in many cases the possibility to isolate the individual multipole terms remains a hard experimental task, because of the difficulty to disentangle the high order magnetic or electric multipole contributions from the dominant resonant terms. For example, in the case of $\mathrm{L}_{3}$ edge of rare earths the magnetic electric dipole contribution $F^{(1)}(E 1-E 1)$, which is associated to the virtual transitions from $2 p \rightarrow 5 d$ states, dominate the scattered intensities, hiding possible high order multipole terms $F^{(2)}$ which are allowed for symmetry. In order to single out the individual components of each multipole through the observation of Bragg forbidden lattice reflections, a dedicated experimental tool have been developed in parallel with the development of the theoretical models for the symmetry analysis of resonant phenomena.

\section{EXPERIMENTAL METHODS}

From the previous section it is clear that in order to discriminate the scattering events summarized in Eq. (2.2), it is necessary to develop an appropriate instrumentation able to deal with the complex relationships between the different interference terms that could be encountered in a diffraction experiment. In this section we will summarize the experimental methods for polarized x-ray diffraction and the advance in instrumentation to study magnetic systems, exploiting the basic properties of 
synchrotron radiation. A typical beamline dedicated to the resonant and $\mathrm{x}$-rays magnetic scattering was ID20 beamline at ESRF, now dismounted, and a general description of the its experimental setup can be found in Ref. [34], and a summary of the scientific applications in Ref. [35].

\subsection{Experimental setups}

In the magnetic and resonant $\mathrm{x}$-ray scattering technique the choice of the scattering geometry and the sample environment is strictly related to the complex relationships between the photon polarization state and the scattering wavevector $\mathbf{Q}$ with respect to the sample orientation, as we have seen in previous Sect. 2. X-ray absorption becomes large at low energy, and then RXS experiments probe a small scattering volume near the surface of the sample. This can be an advantage for single magnetic domain studies, but a severe limitation is introduced in the geometrical scattering condition, due to the reduced Ewald sphere and large scattering angles required to span the available reciprocal lattice space. As a result, most of magnetic and RXS experiments cannot be done in transmission and absorption corrections have to be taken into account.

Two scattering geometries can be used for RXS studies: the vertical scattering geometry, adapted for azimuthal studies, and the horizontal scattering geometry for experiments under extreme conditions, as shown in Figure 1. The azimuthal diffractometer allows the rotation $\hat{\psi}$ of the sample around the scattering vector $\mathbf{Q}$, in order to change the angles between the incident x-ray polarization delivered by the undulators (in general $\hat{\epsilon_{\sigma}}$ ) and the sample crystallographic reference frames, as shown in Figure 1a. The azimuthal method is also used to discriminate the multiple scattering events (the so called "Renninger scans"), to establish the magnetic domain populations and to add an extra geometrical degree of freedom in diffraction experiments. This method is extensively used to discriminate the character and the symmetries of the tensors involved in resonant scattering amplitudes, as explained in Section 2.2.

In the horizontal scattering geometry (Fig. 1b) the incident photon polarization $\hat{\epsilon_{\pi}}$ is parallel to the scattering plane, but could be rotated by a phase plate setup, in order to supply the limitation of azimuthal rotation. The detector arm can also move off plane in the so called "normal beam" geometry, to collect out-of-plane Bragg reflections. This type of diffractometers are specially engineered to hold heavy cryogenic devices like superconducting split-coil magnets, ${ }^{4} \mathrm{He}$ evaporation cryostats, pulsed magnetic field systems.

It is important to remark the necessity to have diffractometers with a high degree of mechanical stability, reproducibility and a sphere of confusion close to the micrometer size. In fact when we deal with high-Q resolution and small single crystals the alignment of samples and the correct set of the UB matrix is primordial. In top of that typical single crystals have Bragg peaks widths of the order of $0.005-0.010^{\circ}$, and if the polarization analyser is used, the alignments become more stringent.

\subsection{X-rays polarimetry}

The main advantage of X-rays with respect to neutrons is that synchrotron radiation produced by planar undulators is completely linearly polarized, with a polarization rate close to $99.9 \%$. The electric field unitary vector $\hat{\boldsymbol{\epsilon}}$ which characterises the X-ray polarization is parallel to the storage ring orbit plane. In general the use of polarized probes (neutron and X-rays) allows to extract more detailed information from the scattering amplitudes encoded in the Bragg intensities. The advantage to have a fully polarized and brillant X-ray source could be exploited to disentangle the different scattering process involved in RXS and NRXS contributions, as we have seen in the Section 2. The different polarization pre-factors contained in the NRXS amplitudes in Eq. (2.4) or in more complex polarization tensors $X^{(p)}$ for the RXS amplitudes in Eq. (2.11) determine scattered intensities containing the peculiar signature of the different characters of scattering process. The combination of site selectivity due to the diffraction conditions and 


\section{Collection SFN}

a)

\section{Vertical}

scattering plane
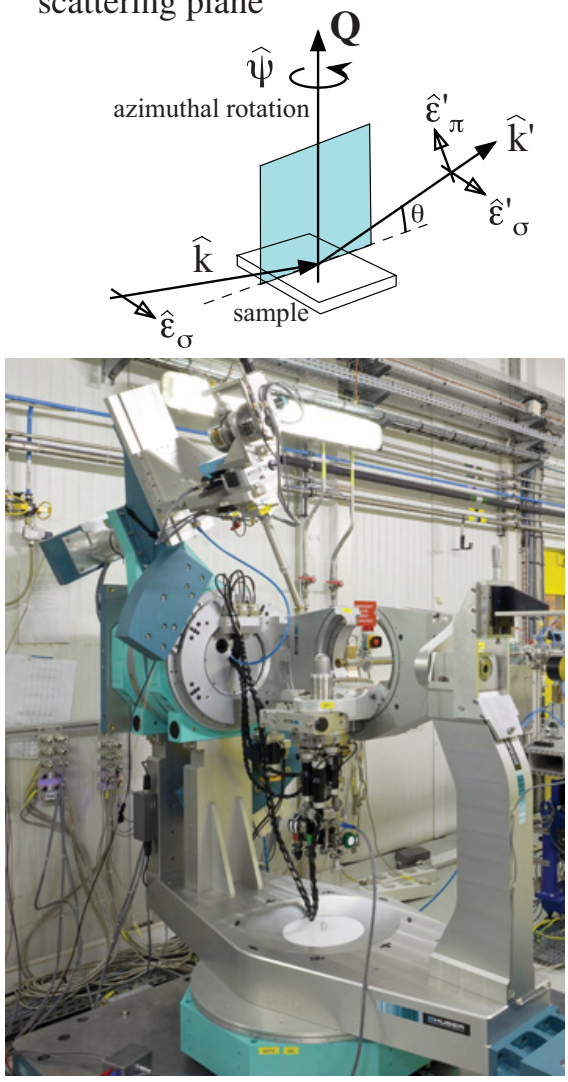

b) Horizontal scattering plane
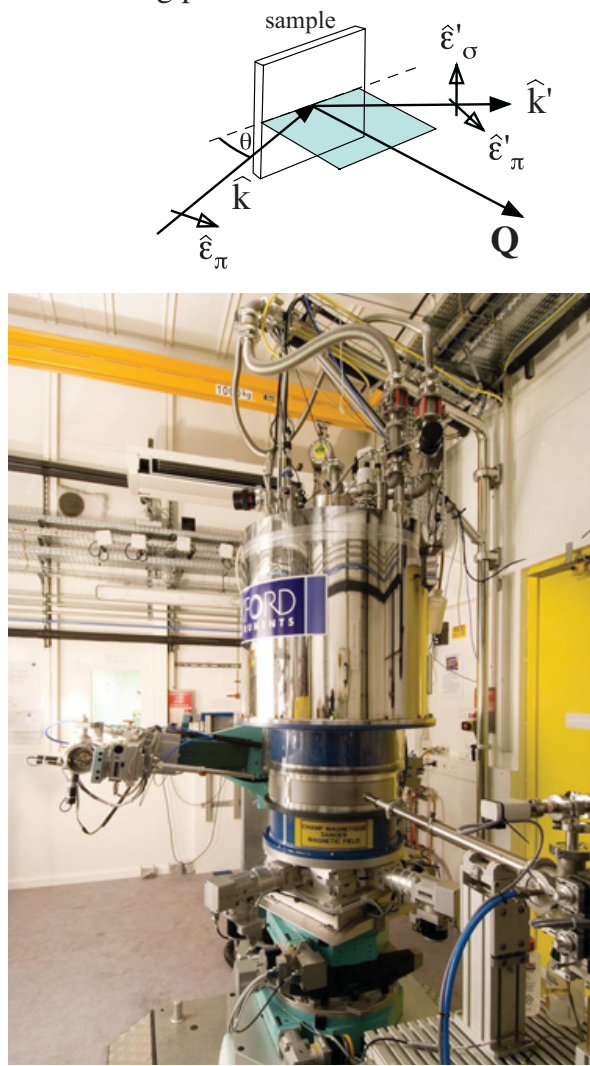

Figure 1. Experimental setup of ID20 magnetic scattering beam line at ESRF. a) Standard four circle diffractometer operating in a vertical scattering geometry. The incident X-ray polarization $\hat{\epsilon}_{\sigma}$ is perpendicular to the scattering plane and the scattered polarization can be analysed in $\sigma-\sigma^{\prime}$ or $\sigma-\pi^{\prime}$ polarization channels. The sample can rotate around the scattering wavevector $\mathbf{Q}$ to perform the azimuthal scans. b) Normal beam six circles diffractometer operating in an horizontal scattering geometry. The incident X-ray polarization $\hat{\epsilon}_{\pi}$ is parallel to the scattering plane. The detector arm can move perpendicular to the scattering plane (normal beam geometry) to detect offplane reflections. In this case corrections to the polarization relationship have to be take into account in the data analysis.

polarization dependence of the scattered beam offer the possibility to extract information encoded in the Bragg reflections, a condition particularly useful when experimental factors limit the access to the Ewald sphere, as in the case of sample environment restrictions for cryostats, magnets and high-pressure cells, or when sample absorption forbids transmission geometry.

\subsubsection{Polarization analysis}

The polarization state of an incident or a scattered $\mathrm{x}$-ray beam is described by a generic unitary vector $\hat{\boldsymbol{\epsilon}}$ or $\hat{\boldsymbol{\epsilon}}^{\prime}$, respectively, parallel to its electric field E. Various orthogonal polarization basis vectors can be choose as a reference system: here we choose an orthogonal basis in which $\hat{\epsilon}_{\sigma}$ is the polarization perpendicular to the scattering plane and $\hat{\epsilon}_{\pi}$ is the polarization lying in the plane, as shown in Figure $2 \mathrm{a}$. 
a)

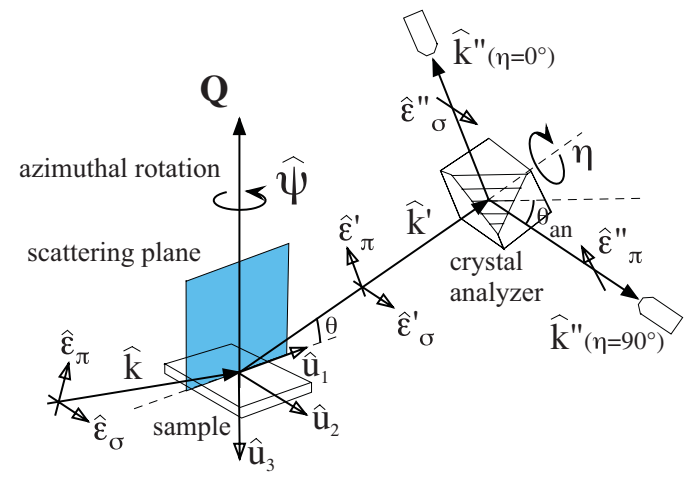

b)

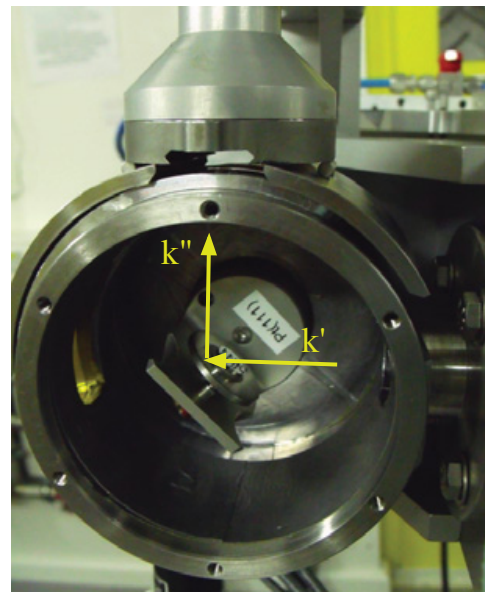

Figure 2. a) Schematic drawing of a general x-ray scattering experiment with the vertical scattering plane and relative photon polarization directions. The unitary vectors $\left(\hat{u}_{1}, \hat{u}_{2}, \hat{u}_{3}\right)$ are used as reference for the laboratory system. b) In-vacuum polarization analyzer setup with a $\mathrm{Pt}(111)$ analyser.

A practical description of the polarization x-rays state is given by the Jones matrix in the basis of $\left[\hat{\epsilon}_{\sigma}, \hat{\epsilon}_{\pi}\right]$, and is valid when we consider a fully polarized beam. A Jones matrix $[\mathbf{A}]$ is a $2 \times 2$ matrix which describe the change of the incident $\hat{\epsilon}$ and scattered $\hat{\epsilon}^{\prime}$ polarizations after the interaction with the physical system, i.e. $\hat{\epsilon}^{\prime}=[\mathbf{A}] \hat{\epsilon}$. For example, in the case of Thomson scattering (see Eq. (2.2)), the scalar product of the incident and scattered x-ray polarizations can be expressed in the form:

$$
\hat{\epsilon}^{\prime} \cdot \hat{\epsilon}=\left[\begin{array}{cc}
\hat{\epsilon}_{\sigma}^{\prime} \cdot \hat{\epsilon}_{\sigma} & \hat{\epsilon}_{\sigma}^{\prime} \cdot \hat{\epsilon}_{\pi} \\
\hat{\epsilon}_{\pi}^{\prime} \cdot \hat{\epsilon}_{\sigma} & \hat{\epsilon}_{\pi}^{\prime} \cdot \hat{\epsilon}_{\pi}
\end{array}\right]=\left[\begin{array}{cc}
1 & 0 \\
0 & \hat{\mathbf{k}}^{\prime} \cdot \hat{\mathbf{k}}
\end{array}\right]=\left[\begin{array}{cc}
1 & 0 \\
0 & \cos 2 \theta
\end{array}\right] .
$$

The polarization factor tells us that, if the incident radiation is purely polarized along one of the basis vectors $\hat{\epsilon}_{\sigma}$ or $\hat{\epsilon}_{\pi}$, then the Thomson scattering does not change its polarization. This properties is used to select the linear polarization of the scattered photons by means of a polarization analyzer device.

The polarization analysis of the scattered beam in the hard $\mathrm{x}$-ray regime is realized through the elastic Bragg diffraction from the polarization analyzer, a high quality single-crystal, whose crystal properties (lattice spacing) are selected to fulfill the diffraction condition with $\theta_{a n}=45^{\circ}$ for the working energy (see Fig. 2). The analyser crystal acts as a polarisation filter for the scattered X-rays because the polarisation term $\left(\hat{\epsilon} " \cdot \hat{\epsilon}^{\prime}\right)$ contained in the Thomson scattering amplitude excludes the rotation of the $\mathrm{X}$-ray polarisation [36]. In addition, it reduces the fluorescence background near the absorption edges and increases the $2 \theta$ angular resolution.

By applying Eq. (3.1) to the analyser crystal (with Bragg angle $\theta_{a n} \approx 45^{\circ}$ ), the Jones matrix for the analyser becomes:

$$
\hat{\epsilon}^{\prime \prime} \cdot \hat{\epsilon}^{\prime}=\left[\begin{array}{cc}
1 & 0 \\
0 & \cos 2 \theta_{a n}
\end{array}\right] \approx\left[\begin{array}{ll}
1 & 0 \\
0 & 0
\end{array}\right]
$$

where $\hat{\epsilon}^{\prime \prime}$ is the photon polarization after diffraction from the analyzer crystal (Fig. 2a).

The polarization analysis, combined with the rotation $\psi$ of the sample about the scattering vector $\mathbf{Q}=\mathbf{k}-\mathbf{k}^{\prime}$ (the so called azimuthal rotation), is the basic method to separate the magnetic or RXS intensities from the charge scattering. 


\section{Collection SFN}

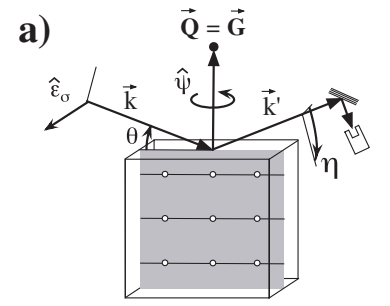

b)
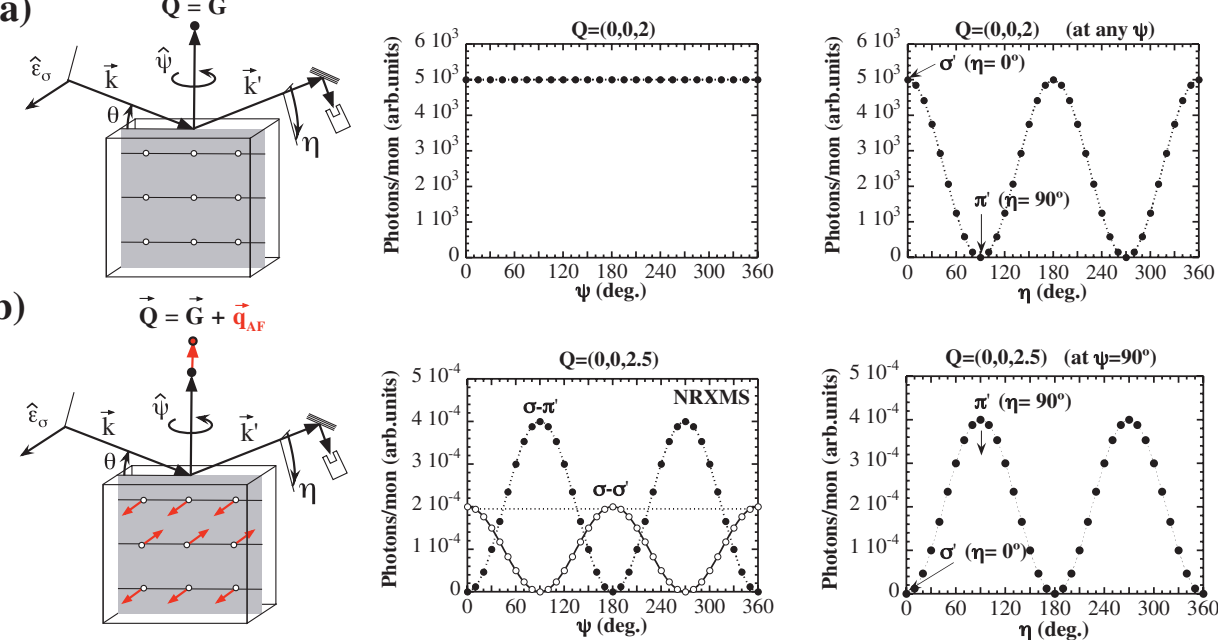

c)
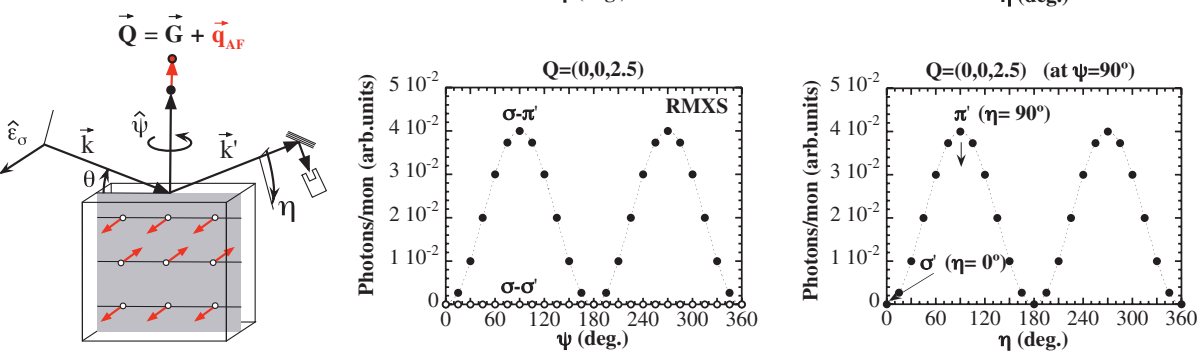

Figure 3. a) Azimuthal scan and linear polarization analysis of a charge peak (002). The incoming x-rays polarization $\epsilon_{\sigma}$ is perpendicular to the vertical scattering plane. The azimuthal scan $\hat{\psi}$ around $\mathbf{Q}=(002)$ has a flat dependence, because the Thomson scattering is isotropic. The charge scattering does not change the scattered $\mathrm{x}$-ray polarization and the x-ray intensity is detected only when $\eta=0^{\circ}$, i.e. in the $\sigma-\sigma^{\prime}$ polarization channel. b) $\mathrm{X}$-ray diffraction of a magnetic reflection $\mathbf{Q}=(0,0,2.5)$. The magnetic moments are oriented perpendicular to the scattering plane when the azimuthal angle $\psi=0^{\circ}$. Both polarization channels $\sigma-\sigma^{\prime}$ (open circles) and $\sigma-\pi^{\prime}$ (black dots) have a sinusoidal dependence which reflects the absence of magnetic component $S_{3}$ along $\mathbf{Q}$. When $\psi=90^{\circ}$ (moment along $\mathbf{u}_{1}$ ) the scattered intensity is recorded when $\eta=90^{\circ}$, i.e. in the $\sigma-\pi^{\prime}$ polarization channel. c) In the resonant regime, and in the case of magnetic dipole transitions $F^{(1)}(E 1-E 1)$, the magnetic intensity of the antiferromagnetic reflection of the previous example has the same azimuthal dependence in the $\sigma-\pi^{\prime}$ channel, but no signal is detected in the $\sigma-\sigma^{\prime}$ channel.

\section{Example: Linear polarization analysis of an antiferromagnetic structure}

Let us consider the linear polarization analysis of a simple antiferromagnetic structure. The polarization analysis of the scattering beam is performed by rotating the crystal analyser around the scattered wavevector $k^{\prime}$ about the angle $\eta$. As expected from the polarization dependence of Thomson scattering amplitude (see Eq. (3.1)), when the incident polarization is $\hat{\epsilon}_{\sigma}$, the scattered intensity is detected only when $\eta=0^{\circ}$ (or $\eta=180^{\circ}$ ). Moreover, because the Thomson scattering (Bragg diffraction) is given by an isotropic charge distribution, then the azimuthal scans do not give any variation of scattered intensities as a function of $\hat{\psi}$, as shown in Figure $3 \mathrm{a}$.

In the case of an antiferromagnetic structure depicted in Figure $3 b$ (we consider a Bravais lattice, with only one magnetic atom per unit cell), the NRMS amplitude given by Eq. (2.4) could be developed by projecting the spin $S_{i}(\mathbf{Q})$ and the orbital $\mathrm{L}_{i}(\mathbf{Q})$ momentum components along the cartesian reference system $\mathbf{u}_{i}$ (see Fig. 2). We can easily see that, considering the relations between the polarization vectors 


\section{JDN 20}

and the scattering wavevectors in Eq. (2.5), the Jones matrix for the magnetic scattering amplitude is:

$$
f^{\text {magn. }} \propto\left[\begin{array}{cc}
S_{2} \sin 2 \theta & -2 \sin ^{2} \theta\left[\cos \theta\left(L_{1}+S_{1}\right)-S_{3} \sin \theta\right] \\
2 \sin ^{2} \theta\left[\cos \theta\left(L_{1}+S_{1}\right)+S_{3} \sin \theta\right] & \sin 2 \theta\left[2 L_{2} \sin ^{2} \theta+S_{2}\right]
\end{array}\right]
$$

In the case of the antiferromagnetic structure shown in Fig. 3-b, where the magnetic moments are perpendicular to the scattering vector $\mathrm{Q}=(0,0,2.5)$, the azimuthal rotation $\hat{\psi}$ produces a sinusoidal modulation of scattered intensities in both the polarization channels. In fact, if we consider only the incident polarization $\hat{\epsilon}_{\sigma}$, the detected intensities in the two polarization channels $\sigma-\sigma^{\prime}$ and $\sigma-\pi^{\prime}$ are proportional to the square of the NRMXS amplitudes:

$$
\begin{aligned}
& f_{\sigma-\sigma^{\prime}}^{\text {magn. }} \propto S_{2} \sin 2 \theta=S \sin 2 \theta \cos \psi \\
& f_{\sigma-\pi^{\prime}}^{m a g n .} \propto 2 \sin ^{2} \theta\left[\cos \theta\left(L_{1}+S_{1}\right)+S_{3} \sin \theta\right]=\cos \theta(L+S) \sin \psi
\end{aligned}
$$

where when $\hat{\psi}=0^{\circ}, S_{1}=L_{1}=S_{3}=0$, and when $\hat{\psi}=90^{\circ}, S_{2}=S_{3}=0$. In this particular simple case the maximum (minimum) of the $\sigma-\pi^{\prime}\left(\sigma-\sigma^{\prime}\right)$ signal corresponds to the rotation $\psi=90^{\circ}$, when the magnetic moments are parallel to the scattering plane.

This scattering configuration is particularly useful in the determination of the magnetic moment orientation or the magnetic S-domain population. Moreover, it can be seen that by taking advantage of the different geometrical prefactors for spin $S$ and orbital $L$ moments, x-ray magnetic scattering provides the possibility to separate spin and orbital moment contributions from the total magnetization density. The problem of discrimination of $\mathrm{L}$ and $\mathrm{S}$ presents a distinct difference from the neutron scattering, where only the total magnetic moment is probed. Moreover, the L/S ratio can be probed in the ordered state, and in particular in antiferromagnetic systems or in modulated magnetic structures. The method of separation of L/S has been applied successfully in simple transition metal oxides $[15,37,38]$, in actinides [39] and in Jahn-Teller cuprates [40].

Similar analysis can be done for the polarization and azimuthal dependence of RXS amplitudes, despite the complications of the tensorial properties of scattering amplitudes, and the number of possible electric transitions associated to the multipole terms, as described in the Par. 2.10. However, it is interesting to consider the magnetic part of the term associated to the electric dipole transition of Eq. (2.10), i.e. the term which includes only the magnetic dipole $F^{(1)}(E 1-E 1)$ in Table 2. The polarization dependence is quite simple in this case because the resonant magnetic $\mathrm{x}$-ray scattering (RMXS) amplitude is given by:

$$
f_{E 1-E 1}^{R M X S} \propto-i F^{(1)}\left[\begin{array}{cc}
0 & z_{1} \cos \theta+z_{3} \sin \theta \\
z_{3} \sin \theta-z_{1} \cos \theta & -z_{2} \sin 2 \theta
\end{array}\right]
$$

where $z_{i}$ are the components projected in the reference frame $u_{i}$ of the unit vector $\hat{\mathbf{z}}$ parallel to the total magnetic moment. Notice that $\hat{\mathbf{z}}$ is just an axial vector parallel to the magnetic moment direction, and does not include any form factor.

In the specific example of Figure 3c, the RMXS amplitudes becomes:

$$
\begin{aligned}
& f_{\sigma-\sigma^{\prime}}^{R M X S}(E 1-E 1) \propto 0 \\
& f_{\sigma-\pi^{\prime}}^{R M X S}(E 1-E 1) \propto z_{3} \sin \theta-z_{1} \cos \theta=-z \cos \theta \sin \psi .
\end{aligned}
$$

This magnetic dipole in general dominate the RXS cross section and is the simplest to calculate. The polarization analysis and the azimuthal scans in this case are very similar to the $\sigma-\pi^{\prime}$ channel of Figure 3b, but no scattering is produced in the $\sigma-\sigma^{\prime}$ channel (the corresponding matrix element is null in Eq. (3.5)). This particular scattering condition (incident $\hat{\epsilon}_{\sigma}$ polarization) is then favorable in order to distinguish the existence of higher order multipoles from the simple magnetic dipole dependence, as we will see in the following examples of Section 4.1. 


\section{Collection SFN}

a)

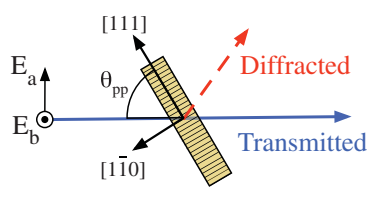

b) Half-wave plate mode $(\Delta \alpha=\pi)$

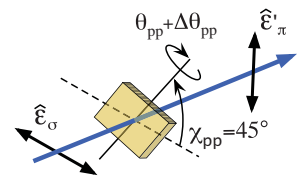

Quarter-wave plate mode $(\Delta \alpha=\pi / 2)$

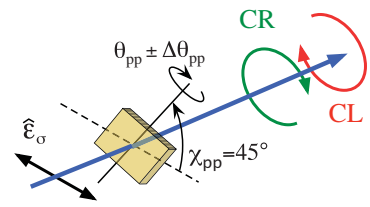

c)

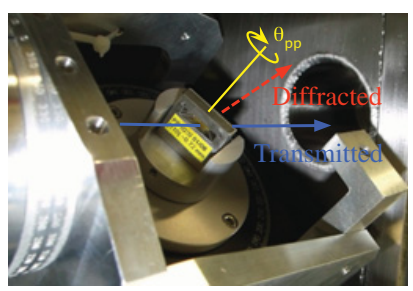

d)

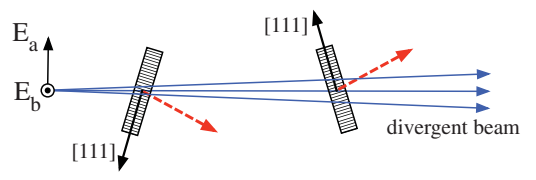

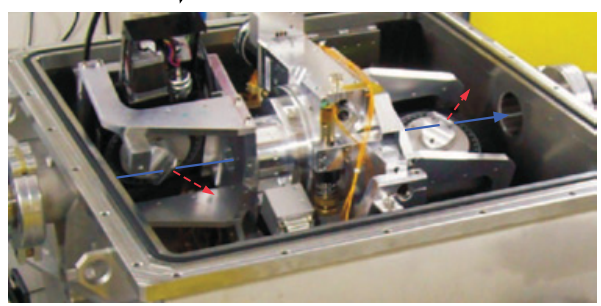

Figure 4. a) Phase plate retarder are optical elements employed to change the polarization of the X-rays by the phase shift between the transmitted and diffracted beam in the dynamical diffraction limit. The incident beam is partially diffracted by the [111] planes of a diamond single crystal, which is cut in a thin slab perpendicular to the [11 10$]$ axis. b) Conversions of incident linear X-ray polarization $\hat{\epsilon}_{\sigma}$ in $\hat{\epsilon}_{\pi}$ linear polarization for a half-wave plate mode $(\Delta \alpha=\pi)$ and in circular right polarisation for the quarter wave plate mode $(\Delta \alpha=\pi / 2)$. Linear polarization scans can be performed by rotating the the $\chi_{p p}$ continuously in half-phase plate mode. c) Picture of the diamond phase plate mounted on the rotation stage inside an in-vacuum chamber. d) Double phase plate setup arrangement to compensate for the off-axis aberration of transmission-type phase retarders [48].

\subsubsection{X-rays phase retarders}

The incident linear light polarization delivered by the planar undulators can be changed (linear or circular) by using an x-ray phase retarder, and the physical origin of this phenomenon is related to the theory of dynamical diffraction in a perfect crystal [41-45].

Different crystalline materials can be used for phase plate retarders. Diamond crystals fulfill the condition of low absorption at the current working energies. Good experimental results are obtained using a thin single crystal of diamond, inserted upstream to the sample, and set in order to excite a given (111) reflection in symmetric Laue geometry. In this case, the diamond slab is cut perpendicular to the (110) axis, as shown in Figure 4a. Alternative methods could be used, as for example in the case of asymmetric Laue geometry with (11)1)-cut. In this case, because the 3-fold symmetry of the crystal surface, one has to take care to avoid exciting one of the equivalent reflections $(\overline{1} \overline{1} 1)$ and $(1 \overline{1} \overline{1})$.

The two X-ray polarization components $\mathrm{E}_{0 a}$ parallel to this scattering plane (polarization $\sigma$ in the diamond reference frame) and $\mathrm{E}_{0 b}$ ( $\pi$ polarization) propagate through the diamond crystal with different refraction index $\left(n_{\sigma}-n_{\pi}\right) \neq 0$, and this difference depends on the departure from the Bragg angle of the incident wave $\Delta \theta$.

When the crystal is slightly detuned from the Bragg position (111), the incident and transmitted waves are then phase shifted with respect to each other, as shown in Figure 4b. The phase plate is oriented in order to form an angle $\chi_{p p}=45^{\circ}$ with respect of the laboratory reference frame, where the incident X-ray polarization is $\hat{\epsilon}_{\sigma}\left(\mathrm{E}_{0 a}=\mathrm{E}_{0 b}\right.$ in the diamond reference frame), as shown in Figure $4 \mathrm{~b}$. In 
this condition, the incident and transmitted polarizations can have a phase difference $\Delta \alpha$ defined as:

$$
\Delta \alpha=\frac{2 \pi}{\lambda}\left(n_{\sigma}-n_{\pi}\right) d=-\frac{\pi}{2}\left[\frac{r_{e}^{2} \lambda^{3} \operatorname{Re}\left(F_{h} F_{\bar{h}}\right) \sin 2 \theta_{p p}}{\pi^{2} V^{2} \Delta \theta_{p p}}\right] d
$$

where $F_{h}$ and $F_{\bar{h}}$ are the complex structure factors for $(h k l)$ and $(\bar{h} \bar{k} \bar{l}), r_{e}$ the classical electron radius, $\mathrm{V}$ the unit cell volume, $\lambda$ the X-rays wavelength, $\theta_{p p}$ the phase plate Bragg angle, $\mathrm{d}$ the beam path in the crystal and $\Delta \theta_{p p}$ the misalignement relative to the Bragg angle $\theta_{p p}$.

Circular polarization is obtained when the phase shift is set to $\pm \lambda / 4$ (quarter wave plate), and it is particularly useful for the study of ferromagnetic and helical magnetic structures.

Linear polarization is obtained when the phase shift is set to $\pm \lambda / 2$ (half wave plate). The plane of polarization is then rotated by twice the angle by which the scattering plane is inclined (angle $\chi_{p p}$ ). By rotating the diamond crystal (set in half wave plate mode) about $\chi_{p p}$, it is possible to change continously the linear polarization direction in order to perform the linear polarization scans. Linear polarization with variable orientation will offer an alternative to the azimuthal dependence, especially in experimental configurations that prohibit an azimuthal rotation of the sample, e.g. when using the cryomagnet, or for surface diffraction, where the azimuth is coupled to the angle of incidence.

The phase plate system is strongly sensitive to the off-axis aberrations, as for example the beam instabilities, the phase shift inhomogeneity due to angular divergence of the beam, the diamond quality. The choice of diamond thickness is directly related to the working energy and must take into account the opposite effects of X-ray absorption and polarization rate. It is therefore desirable to use thin crystals (to minimize absorption), and not to work too close to the Bragg condition, i.e. at $\Delta \theta_{p p}$ much larger than the divergence of the beam and the mosaicity of the crystal. At a given wavelength, one thus has to select a crystal with a large structure factor per unit volume $\left(F_{h} / V\right)$, small mosaicity, and low X-ray absorption. The half wave plate mode is more sensitive to these effects, whereas for quarter wave plate mode it is quite easy to obtain circular polarization rate close to $99 \%$. It is possible to compensate for these effects by using a double phase plate systems composed of two transmission-type phase retarders, as shown in Figure 4d [46]. The off-axis aberrations with the first and the second phase retarders could be canceled out when the two crystals are set in appropriate scattering conditions. An in-vacuum double phase plate setup has been developed and positioned upstream of the diffractometer [48].

When several types of polarization may be mixed up, the polarization state of the beam is better expressed in terms of Poincaré-Stokes parameters, which are conveniently defined in terms of polarization complex vectors $\hat{\boldsymbol{\epsilon}}$. For a transverse electric wave propagating along the z-directions we consider the following terms:

$$
\begin{aligned}
& N_{0}=\left(\epsilon_{\sigma} \epsilon_{\sigma}^{*}+\epsilon_{\pi} \epsilon_{\pi}^{*}\right) \\
& N_{1}=\left(\epsilon_{\sigma} \epsilon_{\sigma}^{*}-\epsilon_{\pi} \epsilon_{\pi}^{*}\right) \\
& N_{2}=-\left(\epsilon_{\sigma} \epsilon_{\pi}^{*}+\epsilon_{\pi} \epsilon_{\sigma}^{*}\right) \\
& N_{3}=\left(\epsilon_{\sigma} \epsilon_{\pi}^{*}-\epsilon_{\pi} \epsilon_{\sigma}^{*}\right)
\end{aligned}
$$

and we define the normalized quantities $\bar{P}_{1}=N_{1} / N_{0}, \bar{P}_{2}=N_{2} / N_{0}$ as the Poincare-Stokes linear polarization rates relative to the azimuthal angles $(0, \pi / 2)$ and $(\pi / 4,3 / 4 \pi)$, respectively, and $\bar{P}_{3}=$ $N_{3} / N_{0}$ is the circular polarization rate. Alternative definition of these parameters can be found in Ref. [7, 11]. The detected beam intensity $\mathrm{I}(\eta)$ can be then expressed in term of $\bar{P}_{i}(\mathrm{i}=1,2,3)$ for any value of $\eta$ as:

$$
I(\eta)=I_{0}\left(1+\bar{P}_{1} \cos 2 \eta+\bar{P}_{2} \sin 2 \eta\right)
$$

where $\bar{P}_{1}=\left[I\left(0^{\circ}\right)-I\left(90^{\circ}\right)\right] /\left[I\left(0^{\circ}\right)+I\left(90^{\circ}\right)\right]$ is the polarization rate parallel and perpendicular to the scattering plane defined by the analyser, $\bar{P}_{2}=\left[I\left(45^{\circ}\right)-I\left(-45^{\circ}\right)\right] /\left[I\left(45^{\circ}\right)+I\left(-45^{\circ}\right)\right]$ the degree 


\section{Collection SFN}

a)

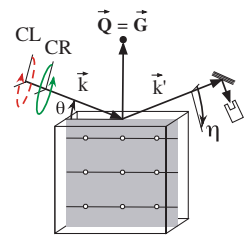

b)

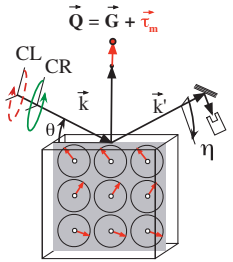

c)

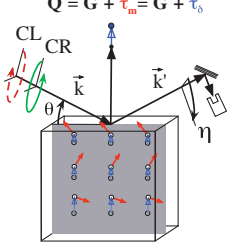

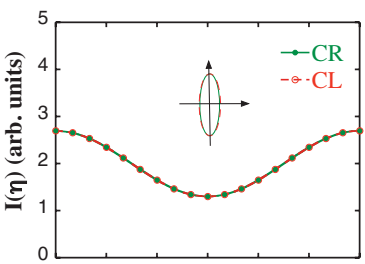
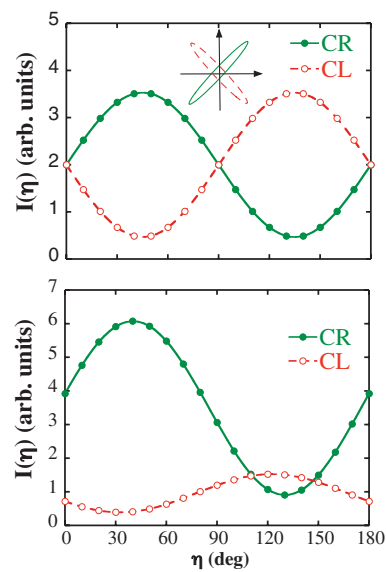
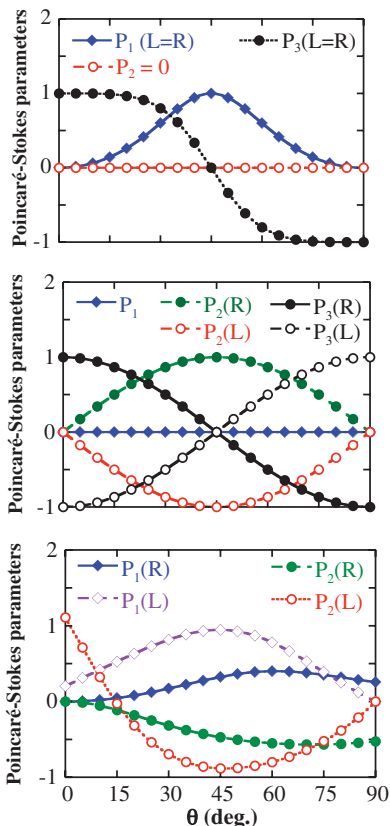

Figure 5. a) Circular polarized $x$-ray diffraction of a Bragg reflection (Thomson scattering). The scattered x-ray intensities have the same $\eta$ dependence for CR (green dots) and CL (red circles). The Poincaré-Stokes parameter $\bar{P}_{2}=0$ and $\bar{P}_{1} \neq 0$, indicating a scattered polarization with a dominant vertical linear polarization. When the scattering angle $\theta=45^{\circ}, \bar{P}_{3}=0$, the scattered x-rays are vertically linear polarized. b) Circular polarized xray magnetic diffraction of a satellite reflection associated to a magnetic cycloid propagating in the direction of the scattering vector $\mathbf{Q}=\mathbf{G}+\tau$. CR and CL polarizations give distinct scattered intensities as a function of the polarization angle $\eta$. In this case $\bar{P}_{1}=0$ and $\bar{P}_{2}= \pm \sin 2 \theta$, indicating a dominant scattered oblique polarization. When $\theta=45^{\circ}, \vec{P}_{3}=0$ and the scattered x-rays are completely obliquely linearily polarized. c) Interference between an incommensurate displacement wave $\tau_{\delta}$ (Thomson) and the magnetic cycloid at the same $\mathbf{Q}\left(\tau_{m}=\tau_{\delta}\right)$. The ratio between the intensities is $t \approx 0.4$. The circular polarized channels are strongly distorted by this interference effect, as it can be deduced from the variation of the Stokes parameters for the CR and CL polarizations.

of oblique polarization. The degree of circular polarization can be determined from the relation $\bar{P}_{3}=\sqrt{1-\left(\bar{P}_{1}^{2}+\bar{P}_{2}^{2}\right)}$, as far as the beam is completely polarized and we can neglect the depolarization effects. Depolarization effects must be taken into account when we use the phase plate retarders to control the incident $\mathrm{x}$-ray polarization, due to the beam divergence and the spectral width, which produce a degradation of the polarization rate.

\section{Example: Circular polarization in collinear cycloidal magnetic structure}

Circular x-rays polarization could be used to study non collinear and incommensurate magnetic systems, such as helical, chiral or cycloidal magnetic structures. Figure 5 shows two examples of polarization analysis of a charge and a magnetic reflections, by using the circular left (CL) and circular right (CR) polarizations (quarter phase plate mode) in non-resonant scattering regime.

Let us consider the case of a circular polarization dependence of Bragg reflection, as shown in Figure 5a. Both left and right circular polarizations give the same scattered polarization dependence $I(\eta)$. In fact, describing the incident circular polarizations as the complex vectors $\hat{\epsilon}_{C R}=\left(\hat{\epsilon}_{\sigma}+i \hat{\epsilon}_{\pi}\right)$ and $\hat{\epsilon}_{C L}=\left(\hat{\epsilon}_{\sigma}-i \hat{\epsilon}_{\pi}\right)$ for the circular right (CR) and left (CL), respectively, and exploiting the Jones matrix 
for the Thomson scattering (Eq. (3.1)), we can calculate the the x-ray scattered polarization as:

$$
\epsilon^{\prime}=\left[\begin{array}{cc}
1 & 0 \\
0 & \cos 2 \theta
\end{array}\right]\left[\begin{array}{c}
1 \\
\pm i
\end{array}\right]=\left[\begin{array}{c}
1 \\
\pm i \cos 2 \theta
\end{array}\right]
$$

and from the Eq. (3.8) we can calculate the Poincaré-Stokes terms $\bar{P}_{i}$ for the Thomson scattered polarization:

$$
\begin{aligned}
& \bar{P}_{1}=\frac{N_{1}}{N_{0}}=\frac{1-\cos ^{2} 2 \theta}{1+\cos ^{2} 2 \theta} \\
& \bar{P}_{2}=\frac{N_{2}}{N_{0}}=0 \\
& \bar{P}_{3}=\frac{N_{3}}{N_{0}}=\frac{2 \cos 2 \theta}{1+\cos ^{2} 2 \theta} .
\end{aligned}
$$

As a result, the $\eta$-dependence of the scattered intensities is as reported in Figure $5 \mathrm{a}$. When the Bragg angle $\theta=45^{\circ}$, the scattered light is completely vertically linearily polarized, because $\bar{P}_{1}=1$ and $\bar{P}_{2}=0$. For different scattering angle, the scattered polarization is elliptical, because a circular contribution $\bar{P}_{3} \neq 0$ is present, but still vertical because $\bar{P}_{2}=0$.

Let us consider now the case of an incommensurate cycloidal magnetic order, described by the magnetic spin moments $\mathbf{S}_{i}=S_{1} \cos \left(\tau \cdot \mathbf{R}_{i}\right) \hat{\mathbf{u}}_{1}+S_{3} \cos \left(\tau \cdot \mathbf{R}_{i}+\pi / 2\right) \hat{\mathbf{u}}_{3}$, i.e. rotating in the same plane and in the direction of the scattering vector $\mathbf{Q}=\mathbf{G}+\tau$, as shown in (Fig. 5b). In general for the incommensurate magnetic structures we need to consider the Fourier series of the modulation $\mathbf{S}_{i}=$ $1 / 2\left(e^{i \tau \cdot \mathbf{R}_{i}} \mathbf{M}+e^{-i \tau \cdot \mathbf{R}_{i}} \mathbf{M}^{*}\right)$ where $\mathbf{M}=S_{1} \hat{\mathbf{u}}_{1}+i S_{3} \hat{\mathbf{u}}_{3}$ (notice that in this case $S_{2}=0$ ). The two terms $\mathbf{M}$ and $\mathbf{M}^{*}$ give rise to two satellite reflections at $\tau$ and $-\tau$, respectively. The non-resonant x-ray scattering differential cross-section is then given by:

$$
\left(\frac{d \sigma}{d \mathbf{\Omega}}\right)^{m a g n .}(\mathbf{Q})=\left|-i \frac{\hbar \omega_{k}}{2 m c^{2}} \sum_{i} e^{i \mathbf{Q} \cdot \mathbf{R}_{i}}\left(e^{i \tau \cdot \mathbf{R}_{i}} \mathbf{M}+e^{-i \tau \cdot \mathbf{R}_{i}} \mathbf{M}^{*}\right) \cdot \mathbf{P}_{S}\right|^{2}
$$

Considering only the polarization dependence of the magnetic satellite $+\tau$, the scattered polarization can be calculated by using the Jones matrix for the non-resonant magnetic x-ray scattering amplitudes (Eq. (3.6)):

$$
\begin{aligned}
\epsilon^{\prime} & =\left[\begin{array}{cc}
0 & -2 \sin ^{2} \theta\left(S_{1} \cos \theta-i S_{3} \sin \theta\right) \\
2 \sin ^{2} \theta\left(S_{1} \cos \theta+i S_{3} \sin \theta\right) & 0
\end{array}\right]\left[\begin{array}{c}
1 \\
\pm i
\end{array}\right]= \\
& =\left[\begin{array}{c}
\mp 2 i \sin ^{2} \theta\left(S_{1} \cos \theta-i S_{3} \sin \theta\right) \\
2 \sin ^{2} \theta\left(S_{1} \cos \theta+i S_{3} \sin \theta\right)
\end{array}\right]=2 \sin ^{2} \theta\left[\begin{array}{c}
\mp\left(i S_{1} \cos \theta+S_{3} \sin \theta\right) \\
S_{1} \cos \theta+i S_{3} \sin \theta
\end{array}\right] .
\end{aligned}
$$

The Poincaré-Stokes can be calculated easily from the $N_{i}$ terms:

$$
\begin{aligned}
& N_{0}=+\left(\epsilon_{\sigma}^{\prime} \epsilon_{\sigma}^{\prime *}+\epsilon_{\pi}^{\prime} \epsilon_{\pi}^{\prime *}\right)=4 \sin ^{2} \theta\left(S_{1}^{2} \cos ^{2} \theta+S_{3}^{2} \sin ^{2} \theta\right)=4 S^{2} \sin ^{2} \theta \\
& N_{1}=+\left(\epsilon_{\sigma}^{\prime} \epsilon_{\sigma}^{\prime *}-\epsilon_{\pi}^{\prime} \epsilon_{\pi}^{\prime *}\right)=0 \\
& N_{2}=-\left(\epsilon_{\sigma}^{\prime} \epsilon_{\pi}^{\prime *}+\epsilon_{\pi}^{\prime} \epsilon_{\sigma}^{\prime *}\right)= \pm 4 S_{1} S_{3} \sin ^{2} \theta \sin 2 \theta= \pm 4 S^{2} \sin ^{2} \theta \sin 2 \theta \\
& N_{3}=\left(\epsilon_{\sigma}^{\prime} \epsilon_{\pi}^{*}-\epsilon_{\pi}^{\prime} \epsilon_{\sigma}^{\prime *}\right)= \pm 2 \sin ^{2} \theta\left(2 S_{1}^{2} \cos ^{2} \theta-2 S_{3}^{2} \sin ^{2} \theta\right)= \pm 4 S^{2} \sin ^{2} \theta \cos 2 \theta
\end{aligned}
$$




\section{Collection SFN}

where we have supposed a pure circular cycloid $\left(S_{1}=S_{2}=S\right)$. The Poincaré-Stokes parameters are given by:

$$
\begin{aligned}
& \bar{P}_{1}=0 \\
& \bar{P}_{2}= \pm \sin 2 \theta \\
& \bar{P}_{3}= \pm \cos 2 \theta .
\end{aligned}
$$

Now the scattered light has a dominant oblique polarization $\bar{P}_{2}= \pm \sin 2 \theta$, with opposite sign for CL and CR, as shown in Figure 5. When the scattering angle is close to $\theta=45^{\circ}$, it become completely linear and oblique $\left(\bar{P}_{2}=1\right.$ and $\left.\bar{P}_{3}=0\right)$. The CL and CR behaviours change symmetrically if the sense of rotation of the cycloid change $\left(\mathbf{M} \rightarrow \mathbf{M}^{*}\right)$ or, equivalently, by considering the opposite magnetic satellite $-\tau$.

Finally, it is interesting to analyze the case in which an interference is produced between the incommensurate cycloidal magnetic structure and a displacement wave at the same propagation vector, for example as a consequence of the application of an external magnetic field (see Sect. 4.2.2) In this particular case the scattered intensities for CR and CL incident polarization interfere and the PoincaréStokes parameters $\bar{P}_{i}$ can be deduced from:

$$
\begin{aligned}
& N_{0}=t^{2}\left(1+\cos ^{2} 2 \theta\right) \pm 2 t(1+\cos 2 \theta) \cos \theta+2 \\
& N_{1}=t^{2}\left(1-\cos ^{2} 2 \theta\right) \pm 2 t(1-\cos 2 \theta) \cos \theta \\
& N_{2}=2 t(1+\cos 2 \theta) \sin \theta \pm 2 \sin 2 \theta
\end{aligned}
$$

where $t$ is the intensity of Thomson scattering with respect to the magnetic scattering. Notice that when $t \rightarrow \infty$ we obtain the case of Figure 5a valid for the Thomson scattering, whereas when $t \rightarrow 0$ the case of Figure $5 \mathrm{~b}$ for the pure magnetic scattering. This scattering technique is particularly useful to study the cycloidal multiferroics, and in particular to determine the magnetic field induced displacement by using the interference between magnetic and charge scattering, as we will see in Section 4.2.

\subsection{Extreme conditions: Low temperatures, high pressures, high magnetic fields, high electric fields}

The development of the extreme conditions at synchrotron radiation facilities remains one of the most promising activities to take advantage of the peculiar characteristic of synchrotron beam: the high brillance, the high collimation and the easy focusing. However, in the domain of magnetic and resonant $\mathrm{x}$-ray diffraction, and in general in most of the photon-in photon-out techniques, the development of low temperatures, high pressures, high magnetic and electric fields is a real challenge for the sample environment design. Indeed, hard $\mathrm{x}$-rays in the enegy range $3.5-20 \mathrm{keV}$ are strongly absorbed by materials, in particular when the working energy is close to the absorption edges. Moreover, the intense photon beam produces heating effects on the sample surface, causing sometimes damages at the sample itself. This poses limitations to the materials which can be used for x-ray windows, and limits the lowest temperatures which can be reached with cryostats.

\subsubsection{Low temperatures}

Most magnetic systems order at low temperatures, and the characterization the important thermodynamic order parameter requires a precise determination of the real temperature at the surface of the sample. The total photon flux impinging the samples inside a cryostat obtained with two undulators can be estimated to be about $10^{13} \mathrm{phot} / \mathrm{sec}$ over a surface area of $500 \times 500 \mu \mathrm{m}^{2}$. At $9 \mathrm{keV}$ this corresponds to a heating power of about $40 \mathrm{~mW}$. As a result, below $5 \mathrm{~K}$ it is necessary to attenuate the beam to reach the lowest temperature phases. The strong absorption of the sample 
surface around the characteristic working energies affects the thermal behavior of measured materials. Indeed, the sample composition (insulating, metallic, superconductive materials), the thermal contacts, the absorption around the characteristic edges, the exchange gas are all important parameters to be taken into account to reach the lowest temperatures. The high photon density at the entrance of the cryogenic equipments prevents the use of the kapton for windows, because it burns very rapidly with consequences on the vacuum performances or implosion of the cryostat itself. To improve the cryogenic performances, special Be domes and windows have been designed for low temperature Displex and cryostat, with different thickness for sample exchange gas $(250 \mu \mathrm{m})$, thermal radiation screen $(250 \mu \mathrm{m})$ or external vacuum insulation $(500-700 \mu \mathrm{m})$.

\subsubsection{High pressures}

The application of high pressures at low temperatures on strongly correlated electron systems leads to variations manifested in the electronic structure. By acting on the lattice parameter, hydrostatic pressure may vary the overlap of orbitals, thus altering the balance of competing magnetic and electronic interactions that are responsible for anomalous low temperature thermodynamic and transport properties, thus playing an essential role in elucidation of physical properties. RMXS could therefore be an ideal technique to be combined with hydrostatic pressure in order to determine structural and electronic order parameters as a function of bond lengths [49]. The development of a hydrostatic pressure technique for resonant magnetic scattering is a difficult challenge because RMXS cross sections is weak and the $\mathrm{x}$-ray absorption is severe in the energy range $5-10 \mathrm{keV}$. A possible solution was undertaken by using Be gaskets, which is highly transparent to x-rays in the exploited energy range [50]. Hydrostatic pressure was created via a moissanite ( $\mathrm{SiC}$ ) anvil cell allowing the possibility of high pressure in combination with large sample volumes, connected to a hydraulic pressure stick compatible with a standard ${ }^{4} \mathrm{He}$ evaporation cryostat (Fig. 6a).

Figure 6d,e show two examples of application of this pressure setup. The first example concerns the RXMS at $\mathrm{Ce}_{3}$ edge of Co-doped $\mathrm{CeFe}_{2}$ single crystal. Notice that the case of $\mathrm{CeFe}_{2}$ was particularly difficult, because of the weakness of the resonance at $\mathrm{Ce}_{3}$ edges and the splitting of the rhombohedral domains which further reduced the scattering intensities. Despite these limitations, the specular magnetic Bragg peak $(5 / 2,5 / 2,5 / 2)$ is clearly detectable up to $17 \mathrm{kBar}$, and the comparison with the charge peaks (222) shows the good hydrostatic conditions which could be obtained with this system [51]. The second example shows the determination of the valence state in SmS through the detection of fluorescence yield at the $\mathrm{Sm} \mathrm{L}_{3}$ edge. In this case the fluorescence yield could be determined more easily. The scattering is particularly intense and the experiment straigthforward. The particularity of this measurement was the possibility to change the pressure at low temperature, because of the re-entrant and irreversible behaviour of this first order phase transition, and to find evidence for the predicted magnetic order by RMXS at $\mathrm{Sm} \mathrm{L}_{3}$ edge in the (100) direction [52].

\subsubsection{High magnetic fields}

Magnetic fields act directly on the exchange interactions between the magnetic elements and allow fine tuning of the delicate balance between different correlation effects. The combination of external applied magnetic field and RXS technique gives unique possibilities to investigate the complex relationships between the correlated microscopic properties of magnetic materials. Magnetic fields influence the domain formation (magnetic annealing, single magnetic domain studies), can induce magnetic phase transitions (metamagnetism, frustrated magnetism in low dimensional magnets), remove the degeneracy of complex magnetic structures, such as multi-k magnetic structures. For spectroscopic applications like magnetic dichroism, the field direction is generally required to be along the beam direction, and X-ray optical access over a relatively small solid angle is sufficient. For diffraction applications, a field 


\section{Collection SFN}

a)

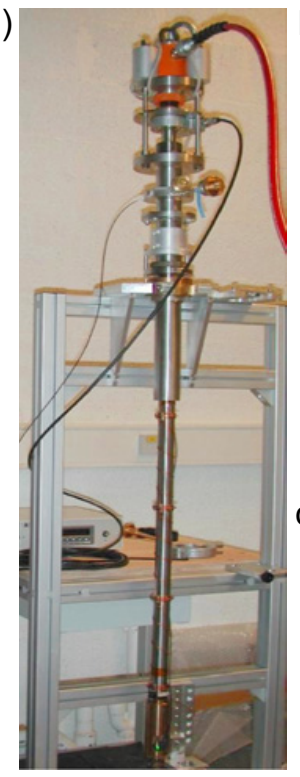

b)

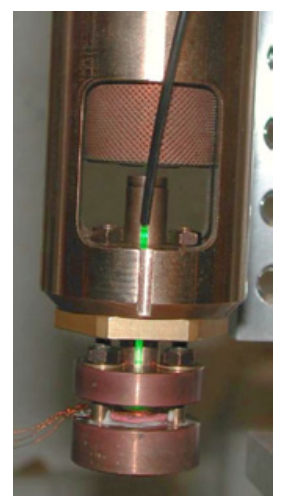

c)

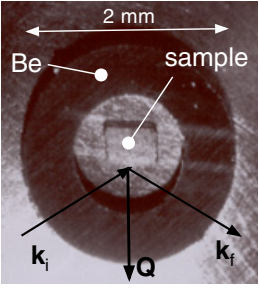

d)

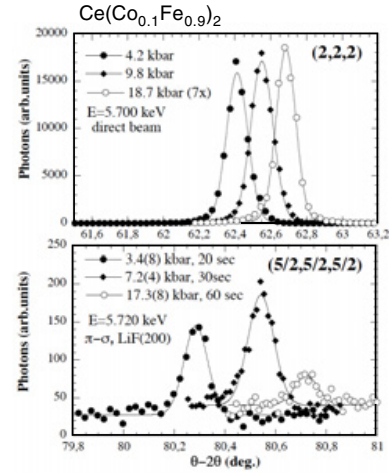

e)

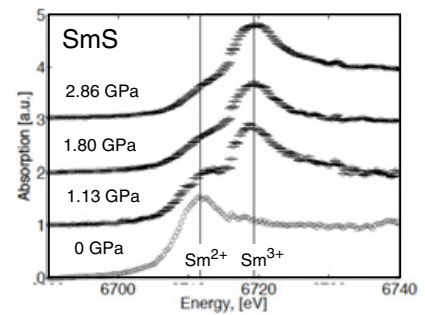

Figure 6. a) Uniaxial hydraulic pressure stick. b) Anvil cell with the green optical fibre for Ruby Luminescence detection, inserted in a solid $\mathrm{Cu}$-Be block of the hydraulic press. (from Ref. [50]) c) Be-gasket and $\mathrm{Ce}\left(\mathrm{Co}_{.1} \mathrm{Fe}_{9}\right)_{2}$ single crystal $\left(80 \times 200 \times 300 \mu \mathrm{m}^{3}\right)$. d) High-pressure resonant magnetic X-ray scattering $(\mathrm{RMXS})$ experiments on $\mathrm{Ce}\left(\mathrm{Co}_{0.1} \mathrm{Fe}_{0.9}\right)_{2}$. Top panel: (222) Bragg reflection as a function of then pressure taken at $100 \mathrm{~K}$. Bottom panel: antiferromagnetic reflection $(5 / 2,5 / 2,5 / 2)$ taken at low temperatures at the $\mathrm{Ce}_{3}$-edge in the $\pi-\sigma$ polarization channel. (from Ref. [51]) e) SmS absorption spectra as a function of increasing pressure above the semiconductor to metal transition, taken at $\mathrm{Sm} \mathrm{L}_{3}$ edge and at $4.5 \mathrm{~K}$. The position of the $\mathrm{Sm}^{2+}$ and $\mathrm{Sm}^{3+}$ white line peaks are indicated (from Ref. [52]).

direction perpendicular to the beam is preferred, and x-ray optical access over a wide solid angle is needed.

As sketched in Figure 7a, the split coil pair design realizes a vertical field of up to $10 \mathrm{~T}$ with a very wide accessible angle in the horizontal plane. This design is unique, and the asymmetric distribution of the wedge spacers and their angular extension limit the top value of the field at $10 \mathrm{~T}$, because of the strong mechanical forces acting between the two magnet split pairs (special reinforcement of the magnet supports limits the magnetic field strength design). The main motivation of this choice was related to the peculiar scattering geometry used in magnetic and RXS, which is restricted to the reflection geometry, and to the wide angle necessary to span the reciprocal space down to the lowest enegies (M-edges of actinides). The homogeneity of the field is about $0.0035 \%$ over $1 \mathrm{~mm}$ and better than $0.1 \%$ over $5 \mathrm{~mm}$ in the sample zone. The vertical access is about $\pm 7^{\circ}$ in order to reach off-plane reflections by the "normal beam" detector arm (see Fig. 7b). The magnet cold bore has an internal diameter of $50 \mathrm{~mm}$ at the sample space, allowing very large sample stick to be accommodated (e.g. a diamond anvil cell for high pressure or electrified stick for electric annealing experiments). The magnet is equipped with a variable temperature insert standard Oxford cryostat, yielding the of 1.8-300 K temperature range at the sample. The cooling power is obtained by using part of the liquid ${ }^{4} \mathrm{He}$ from the main magnet reservoir and evaporating it in the exchanger zone (solid $\mathrm{Cu}$ block) under pumping. The installation of external and internal Be windows (see Fig. 7c) is a necessary improvement for the cryogenic performances of the magnet. 
JDN 20

a)

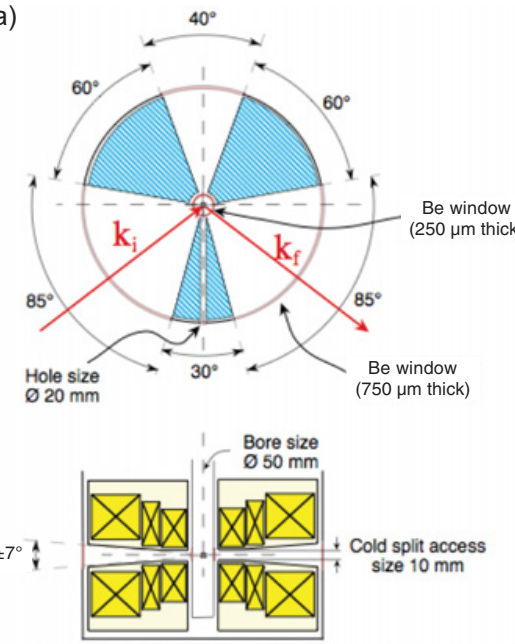

b)

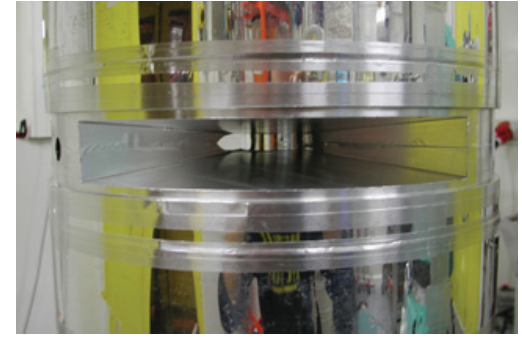

c)

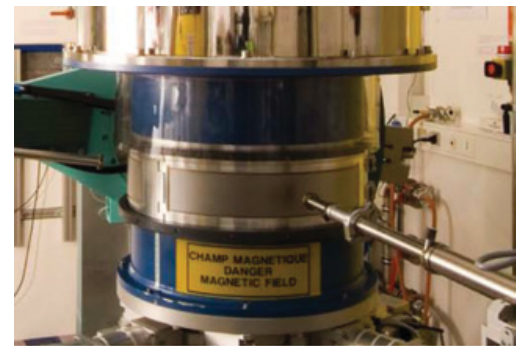

Figure 7. Superconducting vertical split coil magnet of ID20 beam line at ESRF. a) Internal design of the splitpair superconductive $10 \mathrm{~T}$ Oxford magnet, showing the opening angles for X-ray access and the vertical cold split access. Blind zones are marked in blue. b) Internal view of the main opening access. c) External Be windows.

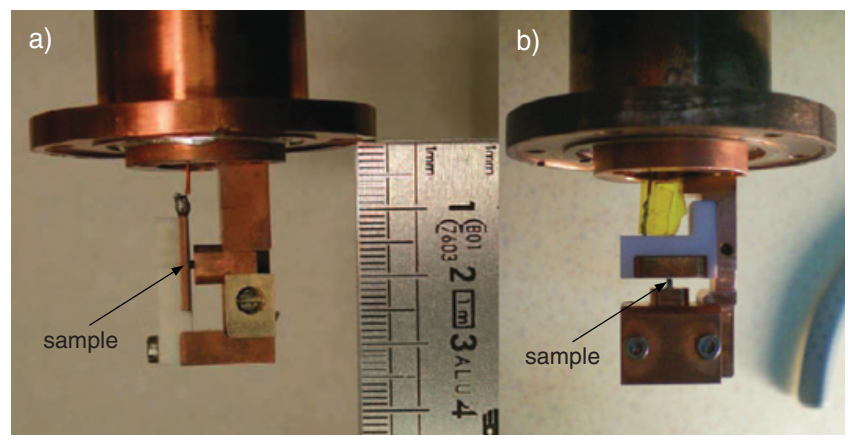

Figure 8. Electric field sample stick head. a) horizontal field orthogonal to the sample surface normal. b) vertical field, orthogonal to the sample surface normal.

\subsubsection{High electric fields}

The application of an electric field on the sample and at low temperatures is an important external parameter to study the novel multiferroics materials. In an electric field experiment the main challenge is to thermalize the sample without producing discharges. A special design of the electric stick head has been chosen, to maximize the thermal contact with the cold sample space bore of magnet or cryostat, as shown in Figure 8. Fields up to $4 \mathrm{kV} / \mathrm{mm}$ have been safely applied at suitably shaped samples. Those two setups leave a completely free scattering surface for the RXS experiment to be performed.

\section{SCIENTIFIC APPLICATIONS}

In the following section we will describe some scientific examples of application of RXS and NRXS, addressed to a selected problems in condensed matter physics, in which synchrotron radiation methods 


\section{Collection SFN}

have been proven to be particularly suited with respect to other complementary techniques. These experiments have been performed at ID20 beam line at ESRF before its closure.

\subsection{RXS in strongly correlated electron systems}

The interplay between charge, orbital and spin degrees of freedom is the key ingredient underlying the physics of transition-metal oxides. An ideal tool for studying the consequences of such a correlation is provided by RXS. The tensorial character of the different RXS amplitudes in the Eq. (2.1) can lead to a breakdown of the extinction rules valid for the crystal structure, giving rise to a forbidden lattice reflections measurable in Bragg diffraction when the photon energy is tuned to the characteristic energy of the atomic system under investigation. In this landscape, forbidden lattice reflections become permitted due to the asphericity of the atomic electron density, giving rise to anomalous tensor susceptibility (ATS) components in the atomic scattering factor.

We applied this technique in two cases of interest: a model system $\mathrm{KCuF}_{3}$ in which we showed that magnetic order is driven by orbital ordering, and that the orbital order parameter acts as a hidden parameter of the magnetic transition. The second case concerns the investigation of the magnetic ground state of $\mathrm{V}_{2} \mathrm{O}_{3}$, in which the analysis of RXS forbidden lattice reflections allows the determination of high order multipoles. The case of RXS on $\mathrm{V}_{2} \mathrm{O}_{3}$ is indicative to describe the complexity of the theoretical interpretation of RXS results but also the potentiality of the RXS methods to study the electronic symmetries of the ground state, an important issue in the physics of strongly correlated electron systems.

\subsubsection{Interplay between orbital and magnetic order in $\mathrm{KCuF}_{3}$}

$\mathrm{KCuF}_{3}$ could be considered an ideal model system for Mott-Hubbard insulators containing transition metal ions, whose magnetic behaviour is largely determined by the structural configuration and the presence of nearly degenerate, partially occupied orbitals [53]. The superexchange Hamiltonian must be generalized to include the orbital degrees of freedom, among the spin one, as derived theoretically by Kugel' and Khomskii (KK), who proposed a model able to describe the magnetic structure of substances containing ions with orbital degeneracy $[54,55] . \mathrm{KCuF}_{3}$ is a quasi-one dimensional spin- $\frac{1}{2}$ magnetic system, which orders antiferromagnetically below $\mathrm{T}_{N}=38 \mathrm{~K}$ with a simple antiferromagnetic structure [56]. The low dimensional magnetic properties derive from the peculiar orbital structure (due to the Jahn-Teller cooperative distortion induced by doubly degenerate $\mathrm{e}_{g}$ orbitals of $\mathrm{Cu}^{2+}$ ) which quenches the ferromagnetic in-plane exchange interaction, as observed in the peculiar magnetic excitations by inelastic neutron scattering experiments $[57,58]$.

The experiment was performed by tuning the incident energy at the $\mathrm{Cu} \mathrm{K}$-absorption edge, as shown in Figure 9. Magnetic reflections in $\mathrm{KCuF}_{3}$ have a magnetic propagation vector $\mathbf{q}_{A F}=(001)$, and appear below $T_{N}$ at the Brillouin zone boundaries. Below the absorption edge (i.e. $\mathrm{E} \sim 8.950 \mathrm{keV}$ ), the polarization analysis of the magnetic reflection (441) shows both the $\sigma-\sigma^{\prime}$ and the $\sigma-\pi^{\prime}$ contributions, as expected for the the NRXMS amplitude, discussed in Section 2.1. Close to the absorption edge, an enhancement of the magnetic intensity appears only in the polarization channel $\sigma-\pi^{\prime}$, and in an extendend energy range (between $8.975 \mathrm{keV}$ and $9.000 \mathrm{keV}$ ). This resonance is attributed to electric dipole $\mathrm{E} 1$ transitions $1 s \rightarrow 4 p$, and it arises from the magnetic dipole $F^{(1)}(\mathrm{E} 1-\mathrm{E} 1)$, because it disappears above $\mathrm{T}_{N}$ (has a magnetic origin) and because the signal is detected in the rotated channel $\sigma-\pi^{\prime}$, as explained in Section 3.2.1 and in Figure 3.

Notice that in the narrow energy window around the pre-edge $(\mathrm{E}=8.975 \mathrm{keV})$ a small resonance appears also in the $\sigma-\pi^{\prime}$ channel, superposed to the NRMS. In general these magnetic resonances are attributed to $1 s \rightarrow 3 d$ quadrupolar electric transitions E2. The sensitivity to magnetic order at the quadrupole threshold has its origin in the spin polarization of the $3 d$ states, whilst at the $1 s \rightarrow 4 p$ dipole transition-energy the resonant enhancement for magnetic reflections is due to the $4 p-3 d$ 
a)

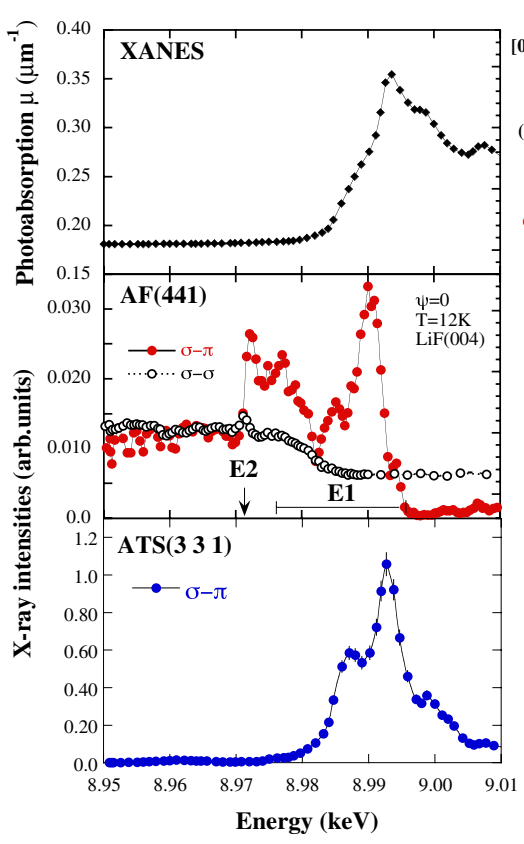

b)

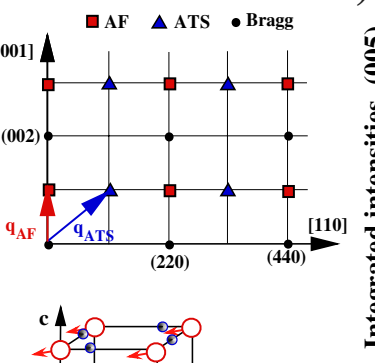

c)
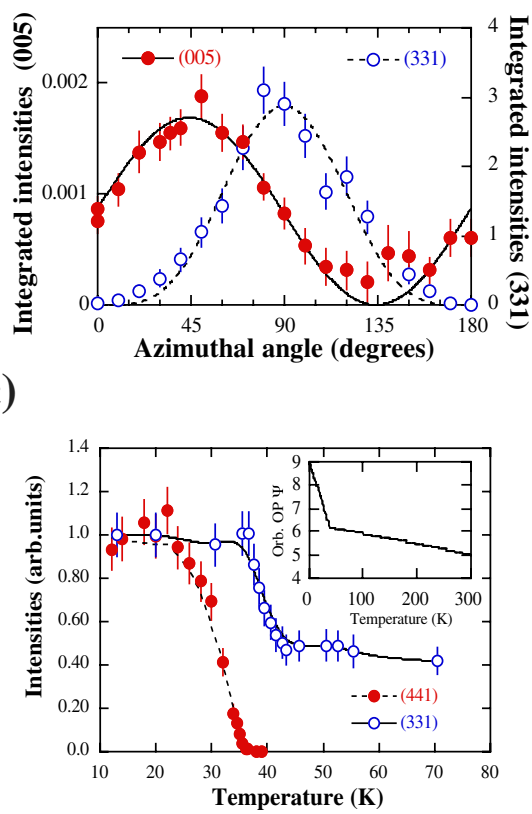

Figure 9. a) Photo-absorption measured by fluorescence yield at K-edge of $\mathrm{Cu}$ (top panel). RMXS of the magnetic AF reflection (441) (middle panel), for $\sigma-\pi^{\prime}$ (black dots) and $\sigma-\sigma^{\prime}$ (open circles) polarization channels. RXS of the ATS reflection (331) (bottom panel) taken in the $\sigma-\pi^{\prime}$. The right panels show the reciprocal scattering plane with the Bragg (black dots) AF (red squares) and ATS (blue triangles) reflections. The $\mathbf{q}_{A F}$ and $\mathbf{q}_{A T S}$ propagation vectors are also indicated. b) Azimuthal dependence of the magnetic (005) and orbital (331) intensities. Data were taken at $12 \mathrm{~K}$ in $\sigma-\pi^{\prime}$ channel using $\mathrm{LiF}(004)$ analyzer, with the incident energy tuned at $\mathrm{E}=8.992 \mathrm{keV}$. c) Temperature dependence of the integrated intensity of the (331) orbital ordering peak (blue circles) and the magnetic (441) Bragg reflection (red circles). The qualitative behavior of the orbital order parameter $\psi$ as a function of temperature, computed in the mean-field approximation, is shown in the inset [60]. (From Ref. [40].)

intra-atomic Coulomb interaction and to the mixing of the $4 p$ with the $3 d$ states of neighbouring $\mathrm{Cu}$ atoms (see Table 1). Tuning the incident energy across the $\mathrm{K}$-edge of $\mathrm{Cu}$, new superlattice reflections were observed at Bragg positions corresponding to a propagation vector $\mathbf{q}_{A T S}=(111)$ (see Fig. 9a). These forbidden reflections become permitted due to the asphericity of the atomic electron density, giving rise to ATS components in the atomic scattering factor. The origin of this scattering is due to the difference in $\mathrm{p}_{x(y)}$ density of states on the two $\mathrm{Cu}$ sublattices. In an indirect way it indicates an alternate occupation of $3 d_{x^{2}-z^{2}}$ and $3 d_{y^{2}-z^{2}} \mathrm{Cu}^{2+}$ hole orbitals, attributed to the Jahn-Teller cooperative distortion which remove the orbital (hole) degeneracy. Weak reflections could be found with the propagation vector $\mathbf{q}_{\text {ATS }}=(110)$, corresponding to an non-alternating orbital arrangement along the c-axis, and the ratio between these two polytypes varies for different samples [56].

Both the magnetic and orbital peaks exhibit an oscillation with two-fold symmetry when the crystal is rotated around the scattering vector, as shown in Figure $9 \mathrm{~b}$. The shift of $45^{\circ}$ between the two curves is a proof that the $\mathrm{Cu}$ magnetic moments are directed along the [110] direction of the pseudo-cubic cell whilst the main contribution to the orbital signal comes from the anisotropy of $\mathrm{p}_{x(y)}$ density of states on the two sublattices.

As $\mathrm{T}$ is lowered, no variations are observed for the charge peaks, neither in intensity nor in position and width. On the other hand, the intensity of the ATS peaks, which is practically constant down to $43 \mathrm{~K}$, 


\section{Collection SFN}
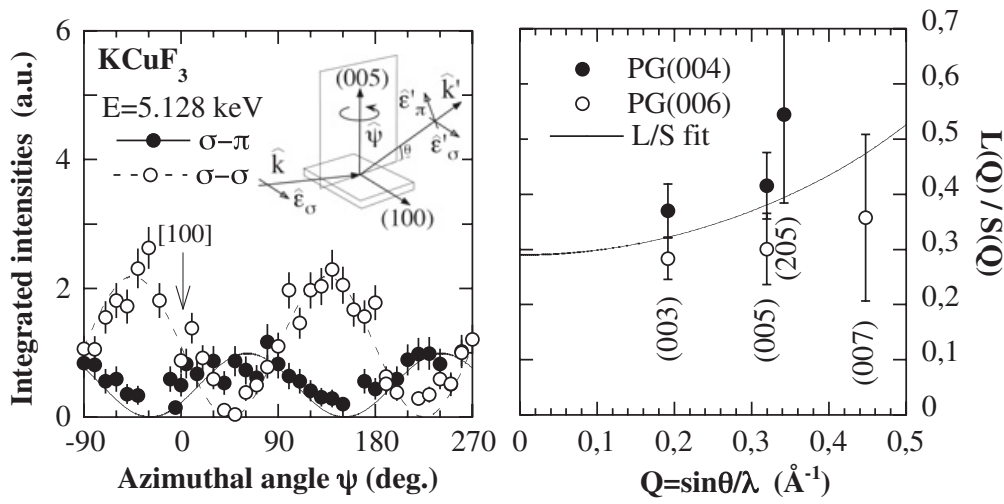

Figure 10. a) Polarization dependence of the azimuthal scan of the AF reflection (005) taken at $\mathrm{T}=11 \mathrm{~K}^{\text {in }} \mathrm{KCuF}_{3}$ well below the absorption $\mathrm{K}$-edge of $\mathrm{Cu}$. b) $\mathrm{L}(\mathrm{Q}) / \mathrm{S}(\mathrm{Q})$ ratio as a function of $\mathrm{Q}$, deduced from NRXMS data. The solid line is a fit to a dipolar model and the extrapolation to $\mathrm{Q}=0$ gives the estimate of the orbital contribution to the Cu magnetic moment $S / L=0.29(5)$. (From Ref. [40]).

increases below this temperature and saturates below $38 \mathrm{~K}$ (Fig. 9c). A similar increase in intensity of the orbital peaks, in a temperature region where the orbital order parameter $\psi$ is expected to be saturated, has been reported near $\mathrm{T}_{N}$ for several manganites [59]. An account for the behavior of $\psi \mathrm{vs} \mathrm{T}$ can be given assuming that the magnetic order is driven by the orbital order, in the sense that the exchange constants between neighbouring atoms are determined by the relative orientation of the occupied orbitals. A simple Landau effective free-energy $F$, which assumes that $\mathrm{T}_{N}$ is determined by the exchange constants and that those are in turn related to $\psi$, can be easily written as a function of $\psi$ and the magnetic order parameter $S$. At high temperature, $\mathrm{T}>\mathrm{T}_{O O}\left(T_{O O}=\right.$ orbital ordering temperature), both $\psi$ and $\mathrm{S}$ are zero. As $\mathrm{T}$ becomes smaller than $\mathrm{T}_{O O}, \psi$ begins to grow, with $\mathrm{S}$ still vanishing until $\mathrm{T}_{N}$ is reached. For $\mathrm{T}<\mathrm{T}_{N}$, also $S$ is non vanishing; the equation determining $\psi$ is then modified, being given by the minimization of $F$ with respect to both order parameters. As shown in the inset of Figure 9c, a steeper rise and a larger saturation value of $\psi$ is obtained as the temperature further decreases below $\mathrm{T}_{N}$, in agreement with the experiment [60]. This precursive transition have been observed in the crystal field excitation by optical method and understood in term of magnetic and exchange-induced dipole mechanisms and vibronic interactions, providing evidence for a symmetry change above the Néel temperature that affects the orbital ordering. [61]

Far from the absorption edge, in the non resonant regime, it is possible to determine the ratio between the orbital $L(\mathbf{Q})$ and the spin $S(\mathbf{Q})$ moments. In the case of vertical scattering geometry and when the magnetic moments are perpendicular to the scattering vector, the expression of NRXMS amplitude in Eq. (2.5) assumes a simple form (see Eq. (3.4)). The azimuthal dependence of the AF reflection (005) has a simple sinusoidal dependence, in which the maxima in the $\sigma-\pi^{\prime}$ (or the minima in $\sigma-\sigma^{\prime}$ ) polarization channel correspond to the condition in which the $\mathrm{Cu}$ magnetic moments are perpendicular both to the $\mathbf{Q}$ vector and to the scattering plane, i.e. they are oriented along the [110] direction, in agreement with RXS data (see Fig. 9b). Notice that the zero intensities in the sinusoidal oscillations insure that a single magnetic domain is probed. From the polarization analysis of different AF reflections it was possible to determine the ratio between the spin and orbital magnetic moments $S / L$, as shown in Figure 10b [40].

Open questions remain to be solved, as for example the role of doping or $\mathrm{OH}^{-}$inclusions, which reflect different electronic behaviour observed in crystal grown with Bridgman method and in which the energy spectra differ from the crystal grown by aqueous solution [62]. These observations suggest that, despite the simplicity of the magnetic and orbital structure, the observed electronic effects appear to be 
a)

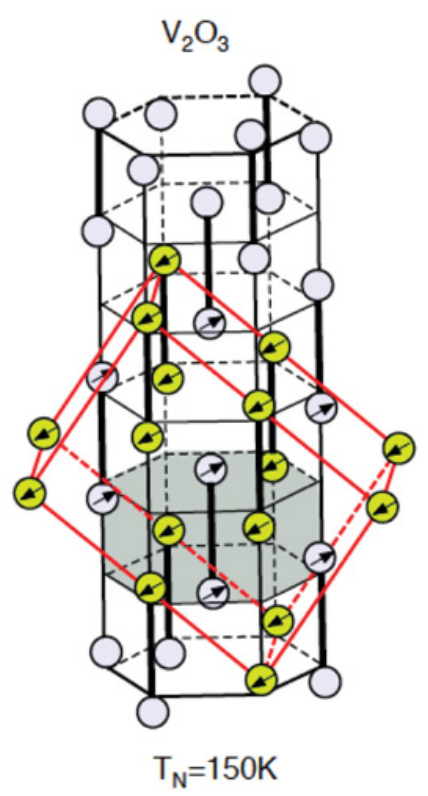

b)

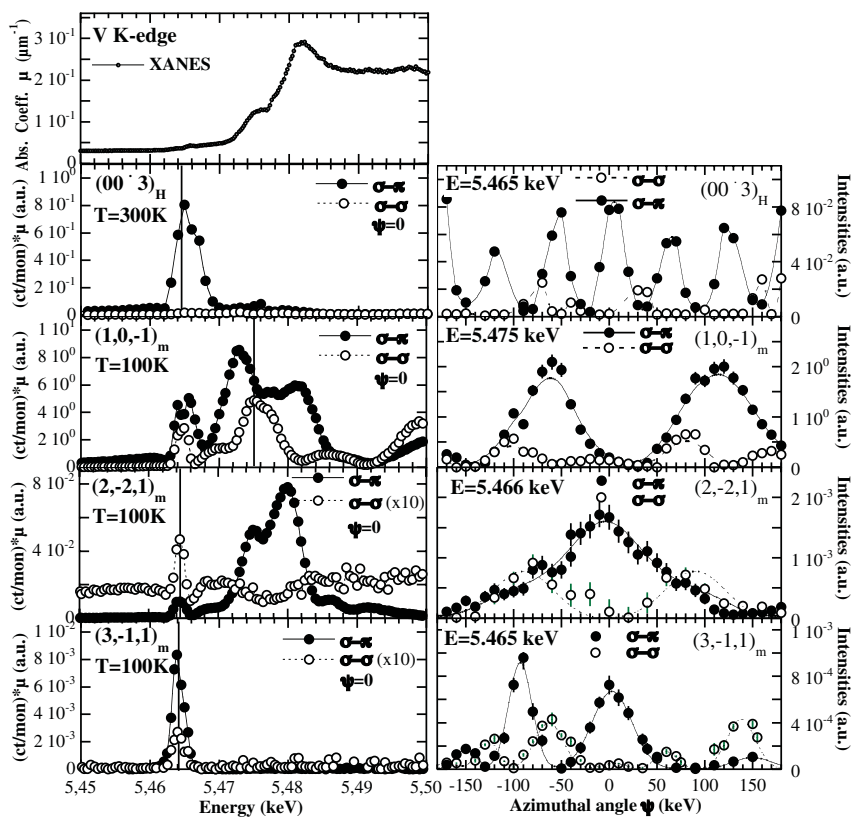

Figure 11. a) Magnetic structure of $\mathrm{V}_{2} \mathrm{O}_{3}$ determined by neutron scattering [64]. The pseudo-hexagonal at room temperature (not primitive) and the monoclinic cells in the AFI phase are also indicated. The circles indicate the $\mathrm{V}$ sites and the arrows the canted magnetic moment directions. b) Energy scans of 2.8\% Cr-doped $\mathrm{V}_{2} \mathrm{O}_{3}$ across the V K-edge (left) and the corresponding azimuthal dependence (right) of different classes of forbidden lattice reflections found at room temperature $(300 \mathrm{~K})$ and in the AFI phase $(\mathrm{T}=100 \mathrm{~K})$. Black (white) dots refer to the $\sigma-\pi^{\prime}\left(\sigma-\sigma^{\prime}\right)$ polarization channels. The vertical thin lines indicate the incident photon energy of azimuthal scans (From Ref. [72].)

strongly sensitive to the small perturbations of static and dynamic local site symmetries, modifying the hybridization between $\mathrm{Cu}$ and $\mathrm{F}$ neighbors.

\subsubsection{High order multipoles in Mott-Hubbard $V_{2} \mathrm{O}_{3}$ by RXS}

$\mathrm{V}_{2} \mathrm{O}_{3}$ is an archetypical Mott-Hubbard metal insulator [63], whose electronic properties remain elusive despite a large program sustained over several decades of experimental and theoretical studies. At room temperature vanadium sesquioxide crystallizes in the $\alpha$-corundum structure as $\alpha-\mathrm{Fe}_{2} \mathrm{O}_{3}$ (space group $\mathrm{R} \overline{3} \mathrm{c}$ ), which can be described with a pseudo-hexagonal space group (Fig. 11a). The stoichiometric compound $\mathrm{V}_{2} \mathrm{O}_{3}$ undergoes a strong volume contraction from paramagnetic metal (PM) to antiferromagnetic insulating state (AFI) at $\mathrm{T}_{N}=150 \mathrm{~K}$, with a monoclinic first order transition (space group I2/a), and a magnetic structure shown in Figure 11a [64]. Chromium doping induces a moderate volume contraction from PI to AFI states below $\mathrm{T}_{N}=180 \mathrm{~K}$ [65]. In 1978 Castellani et al. [66] proposed a theoretical model that explained the magnetic structure in AFI state from the ordering of $\mathrm{V} t_{2 g}$ degenerate orbitals. Our work started in 1999, stimulated by the discussions with the work of theory group at ESRF [67].

A general overview of the energy dependence of all the forbidden lattice reflections observed at $\mathrm{V}$ $\mathrm{K}$-edge are reported in Figure $11 \mathrm{~b}$ as a function of the $\mathrm{x}$-ray polarization and azimuthal dependence. At room temperature forbidden lattice reflections $(00 l)_{H}$ with $l=3(n+1)$ appear at the $\mathrm{V}$ K pre-edge, as shown in Figure $11 \mathrm{~b}$ for the reflection $(003)_{H}$ (defined in the pseudo-hexagonal setting). These 


\section{Collection SFN}

energy scans show well defined resonances in a narrow energy range of about $2 \mathrm{eV}$ around $5.465 \mathrm{keV}$, with the azimuthal scans showing the 3-fold symmetry. This feature is common to all the Corundum sesquioxides as $\alpha-\mathrm{Fe}_{2} \mathrm{O}_{3}$ and $\mathrm{Cr}_{2} \mathrm{O}_{3}$ [28], and these amplitudes have been associated to the $1 s \rightarrow 3 d$ (electric quadrupole transition E2) the electric dipole E1 transitions $1 s \rightarrow 4 p$ being forbidden for this class of reflections [29]. A more detailed analysis based on structure factor calculation and multipole expansion showed that they can be attributed to the parity-even and high order multipoles associated to the electric hexadecapole $\mathrm{F}^{(4)}(\mathrm{E} 2-\mathrm{E} 2)$ and to the parity-odd electric octupole $\mathrm{F}^{(3+)}(\mathrm{E} 1-\mathrm{E} 2)$ due to the lack of inversion symmetry at the V-site [74].

In the low-temperature AFI phase, the 3-fold symmetry is lost due to the monoclinic transition and these forbidden reflections split in three monoclinic domains, which could be indexed (in the monoclinic setting) with the Miller indices $(10 \overline{1})_{m}$ [65]. The energy scans show now a dominant contribution due to the electric quadrupole $\mathrm{F}^{(2+)}(\mathrm{E} 1-\mathrm{E} 1)$ in a broad energy region of about $20 \mathrm{eV}$ around $5.475 \mathrm{keV}$, as shown in the second panel of Figure $11 \mathrm{~b}$. The $\mathrm{F}^{(4)}(\mathrm{E} 2-\mathrm{E} 2)$ and $\mathrm{F}^{(3+)}(\mathrm{E} 1-\mathrm{E} 2)$ contributions are also permitted in the pre-edge, but they are strongly affected by the interference effect due to the $\mathrm{F}^{(2+)}$ (E1E1) contribution. Notice that in $2.8 \% \mathrm{Cr}$-doped samples weak electric quadrupole contribution exists also at room temperature and increases in intensity by approaching $\mathrm{T}_{N}$, an indication of a local loss of "corundum" symmetry in favour of a short-range monoclinic precursive symmetry [75]. Open questions remain on the role of $\mathrm{Cr}$ doping which is responsible for the "moderate" first order transition, destructive in the pure compound, and more generally in the determination of the metal-insulator transition in the paramagnetic phase of $\mathrm{V}_{2} \mathrm{O}_{3}$.

In the AFI state, two new class of reflections appear, with the intensities several orders of magnitude weaker than the previous one, and associated to the magnetic part of the scattering amplitude (i.e. time odd). The first class of reflections are defined by the selection rule $k_{m}-l_{m}=$ odd and $h_{m}=$ even, as for example the $(2 \overline{2} 1)_{m}$ shown in Figure 11, and they correspond to the magnetic reflections found also by neutron scattering [64]. In fact the NRXMS intensity is present far from the absorption edge, as we can see from the low energy part of the spectra (i.e. at $\mathrm{E}=5.450 \mathrm{keV}$ ). The broad resonance observable only in the $\sigma-\pi^{\prime}$ channel is attributed to the magnetic dipole term $\mathrm{F}^{(1)}(\mathrm{E} 1-\mathrm{E} 1)$ and associated to the magnetic polarization of the $4 p \mathrm{~V}$ bands, whereas the pre-edge region contains a resonance centered at $\mathrm{E}=5.465 \mathrm{keV}$ which appears in both the polarization channels $\sigma-\pi^{\prime}$ and $\sigma-\sigma^{\prime}$. This last resonance could contain both the time odd and parity even magnetic dipole $\mathrm{F}^{(1)}(\mathrm{E} 2-\mathrm{E} 2)$ and magnetic octupole $\mathrm{F}^{(3)}(\mathrm{E} 2-\mathrm{E} 2)$, but the analysis of its tensorial character is complicated by the presence of the strong interference with the dominant magnetic dipole term $\mathrm{F}^{(1)}(\mathrm{E} 1-\mathrm{E} 1)$.

The second class of forbidden lattice reflections, with $k_{m}-l_{m}=$ even and $h_{m}=$ odd are visible only when we tune the incident photon energy around the narrow pre-edge, and shows a particular azimuthal dependence. Because of the selection rules, the (E1-E1) dipole transitions associated to the virtual transitions toward the $4 p$ states are forbidden, and only high order multipoles contribute to these classes of reflections, as in the case of the $(3 \overline{3} 1)_{m}$, where the azimuthal dependence shows a peculiar polarization behaviour on both the polarization channels (Fig. 11b, bottom panel).

The observation of these reflections and their interpretation in term of time-reversal and parity breaking symmetries has opened an intense debate during the last years, acting as a driving force to improve theories and experiments. The first interpretation of these reflections as a possible orbital ordering pattern, deduced by the Castellani model [66], was quickly abandoned, because the measure of the L/S ratio in the NRMXS regime showed that the V ions have a spin $\mathrm{S}=1$ [15], instead of $\mathrm{S}=\frac{1}{2}$ as stated by the Castellani model, and in accord with magnetic dichroism experiments [68]. This was a strong indication that in the AFI phase the intra-atomic correlations prevail over band delocalization, contrary to the assumption made by the Castellani model.

A set of theoretical models about the AFI ground state of $\mathrm{V}_{2} \mathrm{O}_{3}$ are based today on a description of a molecular spin state of $\mathrm{V}$ ions of spin $=2$ [69-71]. Further experiments have been performed to shed light on these RXS forbidden reflections, rising some doubts, based by structure factor calculations, 
about the possibility that the observed "orbital" reflections could have a magnetic character, i.e timeodd [72]. Considerations based on the chemical and magnetic space groups, determined previously by neutron diffraction, have led to a different theoretical interpretations about their physical origin: at the beginning Lovesey et al. [73] attributed these resonances to parity-even (E2-E2) multipoles, whereas Tanaka attributed them [71] to a parity-odd (E1-E2) V multipoles. In 2004 Joly et al. [24], using ab initio calculations attempted an interpretation in terms of parity even (E1-E1) and (E2-E2) and parity-odd (E1E2) events. An exaustive calculation made finally by Lovesey et al. [77] reached the conclusion that two dominant effects are present in the multipole expansion: a parity-odd high order multipoles, which include the polar toroidal octupole $\mathrm{F}^{(3-)}(\mathrm{E} 1-\mathrm{E} 2)$ and the polar toroidal dipole $\mathrm{F}^{(1-)}$ (E1-E2), called also anapole, and the parity-even high order multipoles, which include the magnetic octupole $\mathrm{F}^{(3)}(\mathrm{E} 2-\mathrm{E} 2)$ (see Table 2). The advance of calculations and prediction using ab-initio simulations, based on multiple scattering theory and a relativistic extension of the Schrödinger expansion, has open the avenue to a new class of experiments, in which more degrees of freedom have been introduced to disentangle the high order multipole resonances, as for example the use of phase plate polarimetry to change the incident linear polarization and the full analysis of the Stokes parameters of the scattered light [76].

An experiment was designed to isolate and to characterize these classes of high order multipoles, by exploiting all the full polarization capabilities, including the azimuthal scans (see Fig. 12a), a complete polarization analysis of the scattered beam, and by exploiting a phase plate retarder in halfwave plate mode (see Fig. 4b), in order to change the incident linear polarization continuously from $0^{\circ}$ to $180^{\circ}$ (angle $\eta_{i n c}$ in Fig. 12c). Two particular reflections of type $(h 0 l)_{m}$ and $h_{m}=$ odd have been selected, because their structure factors contain a dominant parity odd (E1-E2) contribution, the contribution from parity-even (E2-E2) being absent with a good degree of approximation, as suggested by theoretical predictions [77]. Combining these experimental informations and fitting the collected data to the scattering amplitude derived from the established chemical I2/a and magnetic space group, we have finally provided a direct evidence of the ordering motif of vanadium magnetoelectric multipoles, as shown in Figure 12d. From this analysis the expectation values of $\mathrm{V}$ magnetoelectric multipoles that are time-odd and parity odd for all the values of their rank could be calculated precisely [78].

The study of these contributions by resonant x-ray diffraction is of fundamental importance in current developments of the electronic structure of materials with complex electronic properties, such as magnetoelectricity, piezoelectricity, and ferroelectricity [25]. This work shows the potentialities of RXS to probe directly the multipoles in a crystal with directness of purpose not available with any other experimental method in science of materials.

\subsection{Multiferroics compounds}

The study of multiferroic materials is a rapidly expanding field today thanks to the discovery of a strong coupling between spontaneous long-range magnetic order and ferroelectric order. The big challenge is to find multifunctional materials where multiple physical properties like magnetism, ferroelectricity and ferroelasticity, could be simultaneously exploited in advanced applications in electronics and data storage.

The "multiferroics renaissance" began in 2003, with the discovery by the group of Tokura and Kimura of a magnetic phase in $\mathrm{TbMnO}_{3}$ coexisting with a spontaneous ferroelectric polarization [79]. This compound can be considered to be the progenitor of a class of multiferroics of type-II, in which it is the magnetism itself that produces the ferroelectricity by a particular magnetic mechanism. The intrinsic effect is due to the spin frustration, which produces an incommensurate and non-collinear magnetic order, responsible for the breaking of the magnetic inversion symmetry, and which is accompanied by non-centrosymmetric lattice distortions, responsible for the induced net electric polarization.

Due to the competition between several interactions, the ordered magnetic moments are forced to take non-collinear configuration in which they are canted, and frequently the modulation period of 
a)

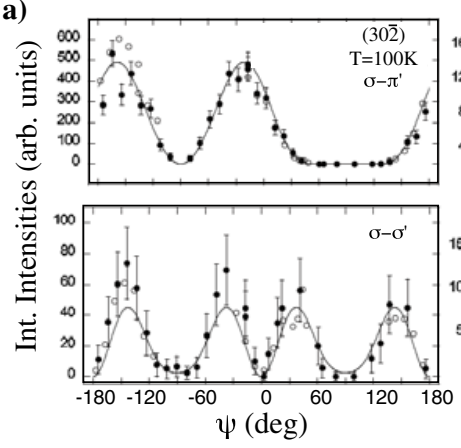

c)

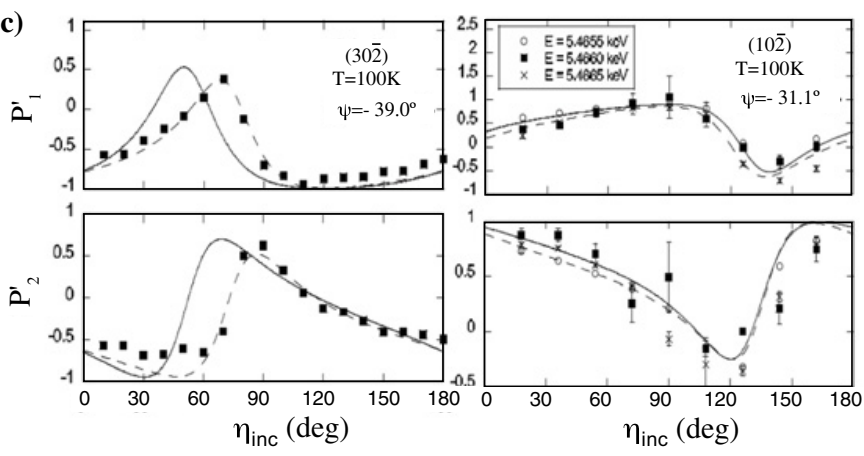

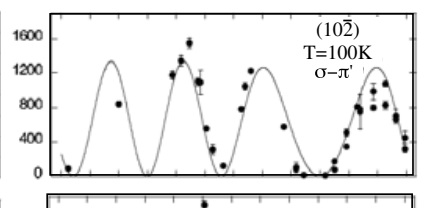

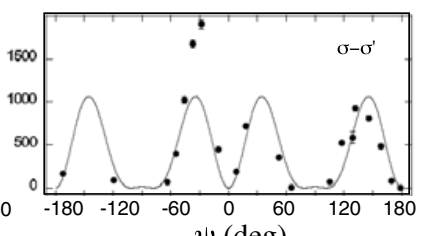

$\psi(\operatorname{deg})$ b)
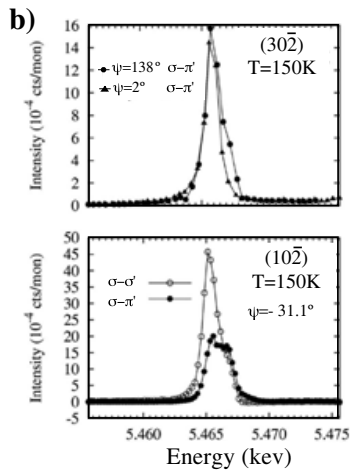

d)

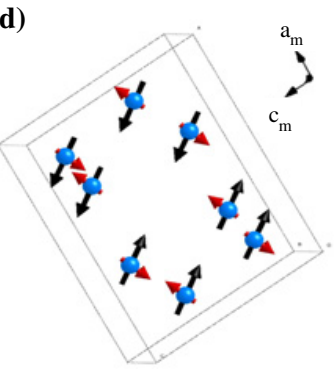

Figure 12. a) Polarization dependence of the azimuthal scans of $(30 \overline{2})_{m}$ and $(10 \overline{2})_{m}$ reflections collected at $\mathrm{T}=100 \mathrm{~K}$ with an incident energy $\mathrm{E}=5.466 \mathrm{keV}$. The origin of the azimuthal angles corresponds to the $(010)_{m}$ reciprocal lattice vector in the scattering plane. b) Measured energy profiles of the $(30 \overline{2})_{m}$ and $(10 \overline{2})_{m}$ reflections at $\mathrm{T}=150 \mathrm{~K}$. c) Stokes parameters $\mathrm{P}_{1}^{\prime}$ and $\mathrm{P}_{2}^{\prime}$ of the secondary beam as a function of the incident linear polarization angle $\eta_{i n c}$ (notice that for $\eta_{i n c}=0^{\circ}\left(90^{\circ}\right)$ the incident polarization is $\sigma(\pi)$ ). Dashed lines correspond to the fitting of the data including the effect of the phase plate depolarization. Continuous line corresponds to the simultaneous fit of polarization data together with azimuthal scans, and including the parameters which contain a dominant $F(E 1-E 2)$ magnetoelectric tensors [78]. d) Positions of the Vanadium ions in the monoclinic unit cell adopted by $\mathrm{V}_{2} \mathrm{O}_{3}$ below the Néel temperature. The red arrows indicate the magnetic moment directions and the black arrows the determined projection of their anapolar moments in the $\mathrm{a}_{m}-\mathrm{c}_{m}$ plane (from Ref. [78]).

magnetic moments does not coincide with the crystallographic periodicity, as shown in Figure 13. These systems are described as spin density waves, where we can define a spin current $\mathbf{j}_{s}=\mathbf{S}_{i} \times \mathbf{S}_{j}$, in analogy with the superfluid current. The electric polarization $\mathbf{P}$ is related to $\mathbf{j}_{s}$ by the relation $\mathbf{P} \propto \gamma \mathbf{e}_{i j} \times \mathbf{j}_{s}$, where $\mathbf{e}_{i j}$ is the vector connecting the magnetic sites $\mathbf{S}_{i}$ and $\mathbf{S}_{j}$, and $\gamma$ is a constant which depends on the spin-orbit coupling and the super-exchange [80]. Different theories based on microscopic [80] or phenomenological [81] approaches have been proposed to explain the origin of the multiferroicity in these compounds, but any direct measurement of the relationship between the magnetic exchange coupling and the ionic displacements has not been possible until now.

In the following sections we present the experimental work in two multiferroics materials, $\mathrm{Ni}_{3} \mathrm{~V}_{2} \mathrm{O}_{8}$ and $\mathrm{TbMnO}_{3}$, in which the onset of a cycloidal magnetic order drives the formation of a ferroelectric state. A key feature in these compounds is the possibility to control the population of magnetic domains (defined by the handedness of the cycloids) by an in-situ electric field. The combination of magnetic nonresonant diffraction by circularly polarised X-rays with the full linear polarimetry of the scattered beam opens the way to a new class of experiments, in which the magnetic order of complex magnetic materials under applied electric and magnetic fields is probed. This technique brings a strong experimental sensitivity to the imbalance in the domain populations, since the handedness of the circular polarisation 
a)

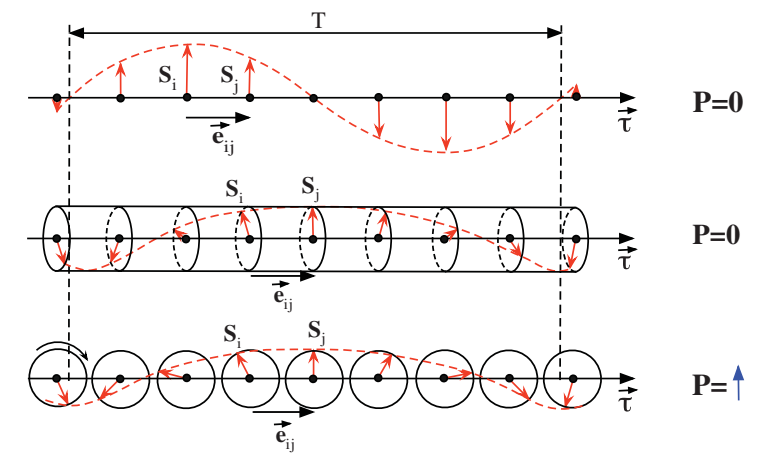

d) clockwise domain

anti-clockwise domain
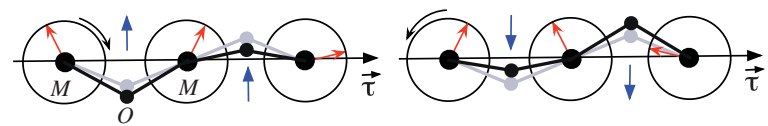

Figure 13. In an incommensurate modulate magnetic structure the periodicity $\mathrm{T}$ of the magnetic moments (red arrows) does not coincide with the crystallographic periodicity. a) A sinusoidal modulated magnetic structure is centro-symmetric and does not produce any electric polarization $\mathbf{P}$. b) In a helicoidal magnetic structure (in most cases) the electric polarization $\mathbf{P}$ is absent, because the spin current $\mathbf{j}_{s}=\mathbf{S}_{i} \times \mathbf{S}_{j}$ is parallel to the helix propagation direction $\mathbf{e}_{i j}$. c) In multiferroics of type-II the cycloidal modulation produces a net electric polarization $\mathbf{P}$ (blue arrow) perpendicular both to the direction of propagation $\mathbf{e}_{i j}$ and to $\mathbf{j}_{s}$. d) The spin-lattice coupling mechanism in a magnetic cycloid is described by the "inverse Dzyaloshinskii-Moriya effect", based on the anti-symmetric exchange, where the oxygen ion displacements produce a net electric polarization (blue arrows). Notice that two magnetic domains exist with opposite polarization directions, defined by the spin rotation (or by the direction of the spin current $\mathbf{j}_{s}$ ).

naturally couples to the sense of rotation of the magnetic moments, leading to an accurate description of the domain state and to the refinement of the magnetic structure.

Circularly polarised XR experiments performed on $\mathrm{TbMnO}_{3}$ allowed to shed more light on the complex magnetic structure of this multiferroic compound, a challenging test case due to its two magnetic sublattices on the $\mathrm{Mn}$ and $\mathrm{Tb}$ sites, by identifying components of the ordering on the $\mathrm{Tb}$ sublattice and phase shifts that earlier neutron diffraction experiments could not resolve. The application of a magnetic field in the ferroelectric phase allowed us to study the oriented displacements of atomic sites at the femtoscale, by exploiting the magnetostriction mechanism. A theoretical analysis of the observed displacements allowed us to shed light on the basic mechanisms responsible for the magnetoelectric coupling in these compounds.

\subsubsection{Imaging of cycloidal domains in $\mathrm{Ni}_{3} \mathrm{~V}_{2} \mathrm{O}_{8}$}

$\mathrm{Ni}_{3} \mathrm{~V}_{2} \mathrm{O}_{8}$ is an example of a magnetoelectric multiferroic in which magnetism and ferroelectricity not only coexist, but are coupled together. These materials present an interesting opportunity for technological applications, but this is reliant on overcoming an outstanding challenge, namely understanding the evolution of the domain states in external applied magnetic fields, and our work presented here is a first step in this direction.

Circularly polarized XR naturally couple to the handedness of spin cycloids, and through a complete Stokes polarimetry analysis of the scattered beam it is possible to identify information on magnetic structures that is typically inaccessible through neutron diffraction experiments. In the case of $\mathrm{Ni}_{3} \mathrm{~V}_{2} \mathrm{O}_{8}$, the method not only facilitated the refinement of the magnetic structure, but also allowed real space 


\section{Collection SFN}
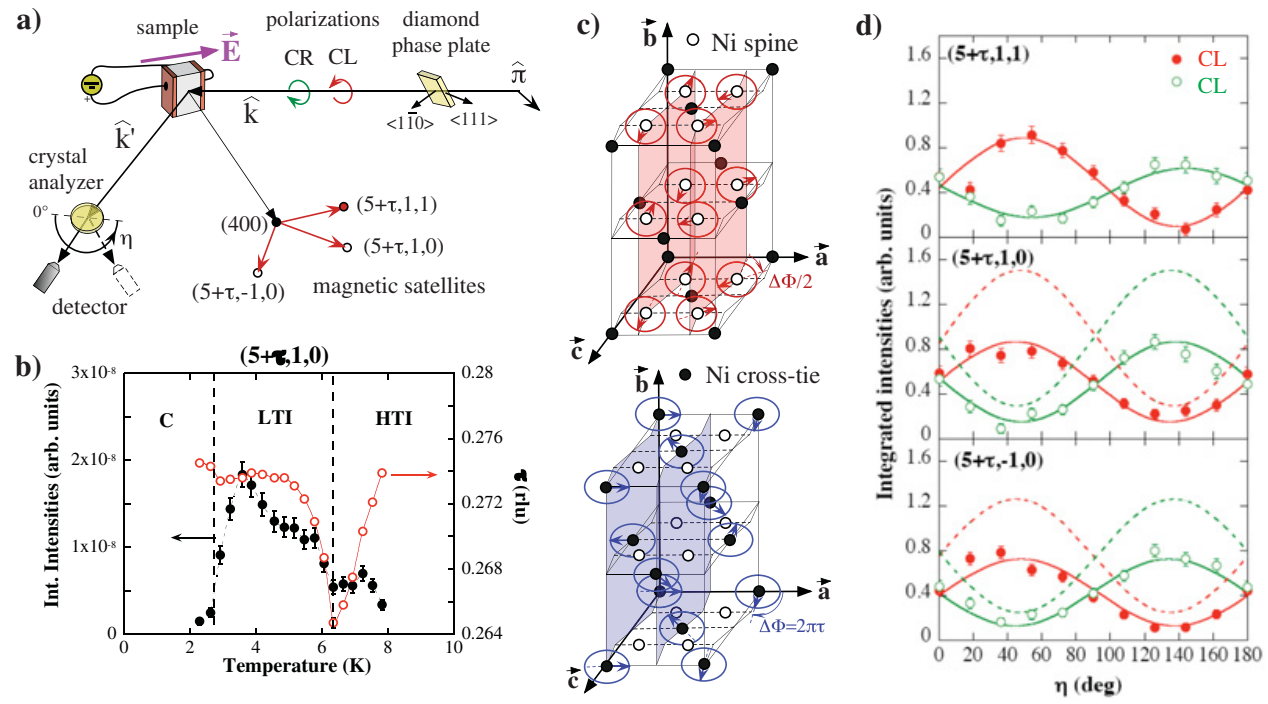

Figure 14. a) Experimental setup used to measure the magnetic reflections in $\mathrm{Ni}_{3} \mathrm{~V}_{2} \mathrm{O}_{8}$. A diamond quarter wave plate converts the incident linear polarization $\hat{\pi}$ in circular left (CL, red) and circular right (CR, green) polarizations. A crystal analyser selects the final polarization state by rotating around the scattered wavevector k' about the angle $\eta$. b) Temperature dependence of the intensity and propagation vector $\tau$ of the magnetic reflection $(5+\tau, 1,0)$ in the low temperature phases. In the low temperature incommensurate phase (LTI) a cycloidal ferroelectric order is established. c) Magnetic structure of $\mathrm{Ni}_{3} \mathrm{~V}_{2} \mathrm{O}_{8}$ associated to the Ni magnetic moments in the "spine" (top panel) and "cross-tie" sites. d) Polarization dependence of magnetic satellites as a function of the incident circular polarization. The continuous lines are deduced by taking into account only the "spine" Ni moments whereas the broken lines are calculated with the neutron scattering model (from Ref. [82]).

images of the magnetic cycloidal domains to be obtained. Their evolution is followed as they are controlled via the magneto-electric coupling by the applied electric field, and cycled through a hysteresis loop, thus collecting valuable information on domain formation, inhomogeneities and domain wall movement.

The XR incident polarization, normally linear and parallel to the scattering plane (polarization $\pi$ ), can be modified in circular right (CR) or circular left (CL) polarizations by a diamond quarter wave plate, with an efficiency close to $99 \%$ (see Fig. 14a). A polarization analyser in used to determine the Stokes-Poincaré parameters of the scattered beam.

In $\mathrm{Ni}_{3} \mathrm{~V}_{2} \mathrm{O}_{8}$ there is a low temperature incommensurate (LTI) phase between $2.8 \mathrm{~K}$ and $6.3 \mathrm{~K}$ that is accompanied by the onset of a finite spontaneous electric polarization, as shown in Figure 14b [85].

Based on neutron diffraction experiments it was proposed that this LTI phase corresponded to a cycloidal order of the spins on two different symmetry nickel sites "spine" and "cross-tie", as shown in Figure 14c [84]. These two sites could be selectively probed by taking advantage of the magnetic structure factor. Indeed, magnetic reflections can be classified according to their indices (hkl): in type-1 reflections $(\mathrm{h}=$ odd, $\mathrm{k}=$ odd, $\mathrm{l}=$ odd $)$, cross-tie moments cancel such that only the spine site moments contribute; in type- 2 reflections $(\mathrm{h}=$ even, $\mathrm{k}=$ even, $\mathrm{l}=$ odd), the reverse is true, such that the crosstie moments are singled out; in type- 3 reflections $(\mathrm{h}=$ odd, $\mathrm{k}=$ odd, $\mathrm{l}=$ even $)$, both moment types contribute to the scattering.

The sample was mounted such that the spin cycloid lays in the horizontal scattering plane, which resulted in the polarization state of the scattered beam being strongly sensitive to the domain populations, and cooled down under an electric field, in order to obtain a single magnetic domain. 

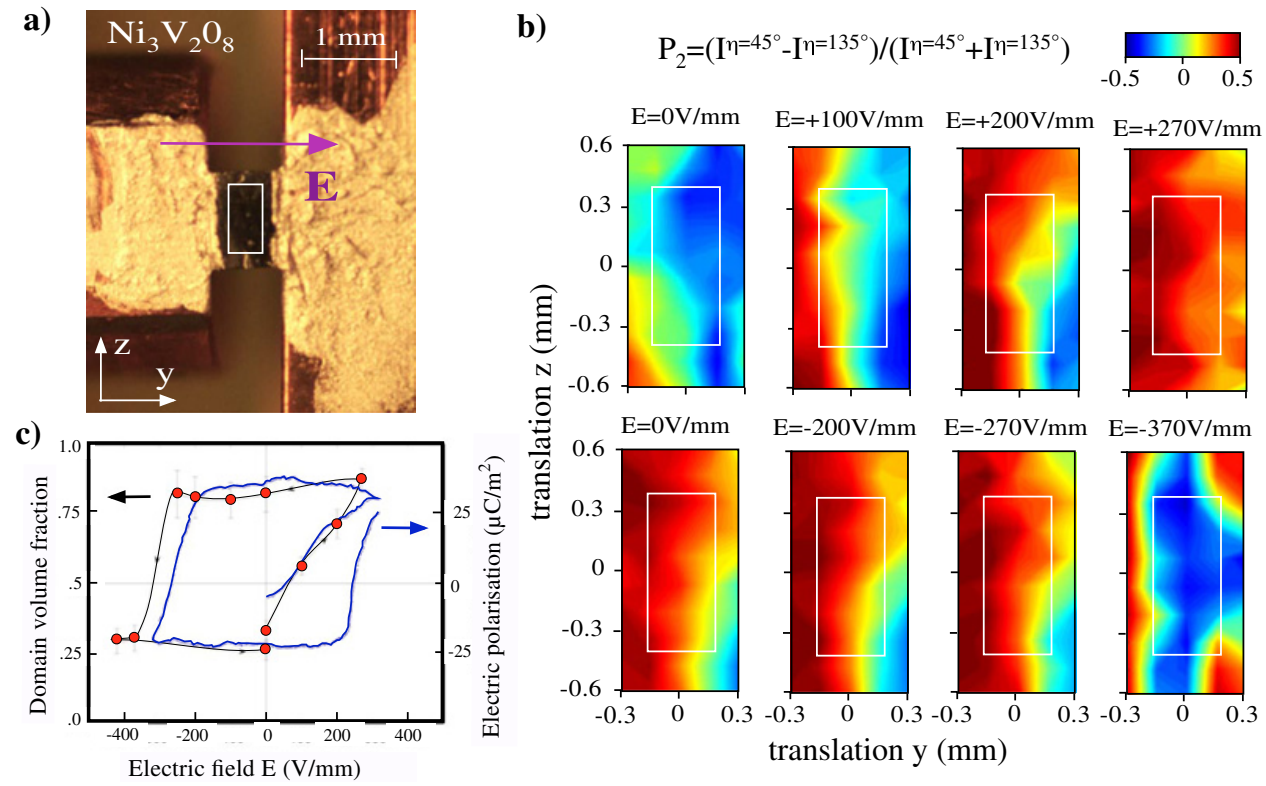

Figure 15. a) Picture of the $\mathrm{Ni}_{3} \mathrm{~V}_{2} \mathrm{O}_{8}$ single crystal placed between two electrodes. b) Topographic intensity maps obtained by circularly polarized XR diffraction on a magnetic satellite reflection $(5+\tau, 1,0)$ in $\mathrm{Ni}_{3} \mathrm{~V}_{2} \mathrm{O}_{8}$ as a function of the applied electric field. The intensity profile $\mathrm{P}_{2}$ is defined as the Stokes-Poincaré parameter associated with the diffracted oblique linear polarization and it is proportional to the cycloidal domain population. The colours represent the percentage of the clockwise and anti-clockwise magnetic domain population. b) Comparison between the magnetic domain population hysteresis loop (red dots), averaged over the central sample area (white square), and the bulk measurements of the electric polarization (blue dotted curve) (from Ref. [82]).

Figure $14 d$ shows the polarization analysis of two class reflections, type- $1(5+\tau, 1,1)$ in which only the spine moments contribute, and type-3 $(5+\tau, 1,0)$ which include both spine and cross sites. Starting with the pure spine type- 1 only reflection $(5+\tau, 1,1)$, we have compared our data for both CL and CR with a calculation for the magnetic structure proposed in an earlier neutron-diffraction study, which include both the Ni site ordered [84]. These experimental results show unambigously that the Ni magnetic moment on the cross-tie sites remains disordered. As a results, the onset of ferroelectricity is associated with the cycloidal ordering of the moments on the spine sites only.

From Figure 14d we can also deduce that the scattering process converts quite completely the incident circular polarization state into a linear oblique polarization state inclined at $45^{\circ}$ with respect to the scattering plane, and described by the Stokes parameter $\bar{P}_{2}$, as defined in Eq. (3.9). By determining the value of $\mathrm{P}_{2}$ at different points over the sample surface it is then possible to extract the domain populations.

To establish the evolution of the domains as a function of E field, the sample was initially cooled to $5 \mathrm{~K}$ with no voltage across the sample. The incident beam spot was then reduced in size to $\left(250 \times 380 \mu \mathrm{m}^{2}\right)$ to maximize the resolution while still giving a reasonable count rate in the detector. The sample was rastered through the beam measuring the value of $\mathrm{P}_{2}$ at each spot and hence finding the percentage of left- and right-handed magnetic cycloidal domains. Then a voltage was applied whilst remaining at $5 \mathrm{~K}$, before repeating the measurements of $\mathrm{P}_{2}$. Figure 15 reveals that this method allowed us to resolve inhomogeneities in the domain populations with a good resolution. The evolution of the domain populations is gradual, with the boundary between the two shifting as a function of applied electric field, as opposed to the nucleation of one domain within the other, which would lead to a 


\section{Collection SFN}

more randomnized distribution. After a complete hysteresis loop, the domains become pinned close to the edges of the sample, an indication of a presence of finite size effects, due to self-induced demagnetization or depolarization fields, and driven by the need to minimize surface energy. By averaging the domain populations over the central area (unaffected by the closure domains) it is possible to extract a magnetic domain population hysteresis loop, and compare it with electric polarization measurements (Fig. 15c). The excellent agreement indicates that by imaging the magnetic domains in multiferroic $\mathrm{Ni}_{3} \mathrm{~V}_{2} \mathrm{O}_{8}$ we are in effect also imaging the ferroelectric domains.

The demonstration of the possibility to image the multiferroic domains in $\mathrm{Ni}_{3} \mathrm{~V}_{2} \mathrm{O}_{8}$ opens up the prospect to use this technique to image the evolution of magnetoelectric domains under the effect of applied electric field in operational devices, to study memory effects within hysteresis loops, and, on optimising the x-ray focusing on a micrometer scale, to analyse the mesoscopic structure of the domain walls [86].

\subsubsection{Circularly polarized x-ray magnetic scattering experiments in $\mathrm{TbMnO}_{3}$}

$\mathrm{TbMnO}_{3}$ is the canonical magnetoelectric multiferroic, in which the phase transition to a non-collinear cycloidal spin arrangement at $\mathrm{T}=27 \mathrm{~K}$ is concomitant with the onset of electric polarisation as a result of the breaking of inversion symmetry [79]. The experimental setup is similar to that shown in Sect. 4.2.1, where the sample was cooled down to $\mathrm{T}=15 \mathrm{~K}$ in a superconducting magnet with an applied electric field in the b direction (see Fig. 16a). We have investigated the ferroelectric (FE) phase in which an incommensurate (IC) cycloidal phase is formed at Mn site, as shown in the magnetic phase diagram of Figure 16b.

Four magnetic satellite reflections $(4, \pm \tau, \pm 1)$ corresponding to the incommensurate magnetic modulation $\left(\tau=0.28 \mathrm{rlu}\right.$ ) of $\mathrm{TbMnO}_{3}$ are detected as a function of the crystal polarisation analyzer angle $\eta$, discriminating the scattered XR polarization, as shown in Figure 16a. Because of the coupling between the handedness of the magnetic cycloid and the circular XR polarizations, it is possible to distinguish the population of the magnetic domains, associated with the sense of rotation of the magnetic cycloid.

We established our experimental sensitivity to the imbalance in the magnetic domain populations produced by cooling down the sample with opposite electric field E polarities, by demonstrating the complementary behaviour of the intensities of the four magnetic satellites for circular left CL and CR polarizations depending on the sign of $\tau$, as shown in Figure 16a. This differs strongly from the expected results for equi-populated domains in which case the signal should be very similar for CL (red dots) and CR (green dots). Notice that the magnetic satellite intensities change also with the sign of the magnetic propagation vector $\tau$, whereas it does not for the incident linear polarization $\pi$ (black dots). By switching the electric field, the CL and CR polarizations produce a reversal behaviour on the magnetic satellite intensities, because the magnetic cycloid changes its sense of rotation. This is well shown in Figure 16c, where the scattered x-rays polarization is analyzed in detail for the magnetic satellite $(4,-\tau,-1)$ as a function of two opposite electric fields. Finally, by rotating the crystal polarization analyser around the scattered wavevector $\mathbf{k}^{\prime}$, the Stokes-Poincaré components of the diffracted XR could be determined, and the detailed information encoded in the magnetic satellites could be disentangled.

Whilst typically magnetic structure determination involves refining the intensity data for as wide a range of magnetic satellites as possible, instead we took advantage of the scattering cross-section for non-resonant X-ray magnetic scattering, in which the coupling of the polarisation state and the experimental geometry enables the magnetic structure to be refined just using the full polarization dependence of only four satellite magnetic reflections. The technique not only provides unique insight into the formation of cycloidal domains, leading to a quantitative description of the domain state, but also allows the refinement of the magnetic structure obtained from an earlier neutron diffraction study [90]. In particular, the absolute sense of rotation and the phase shifts of individual magnetic sublattices 
a)

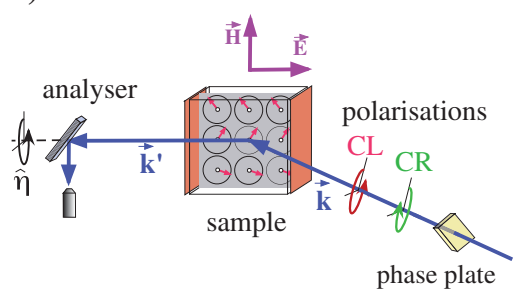

c)

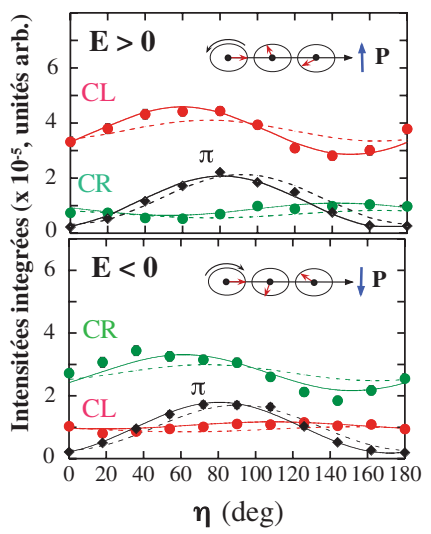

b)

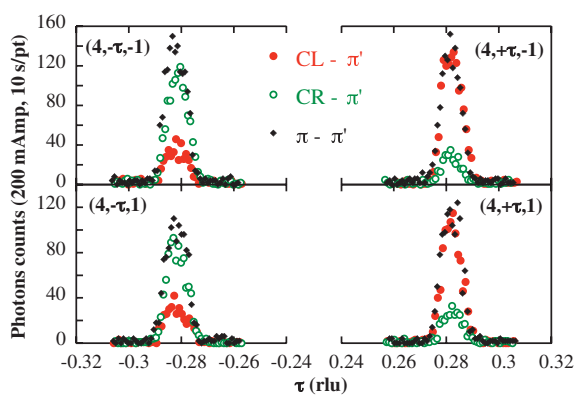

d)

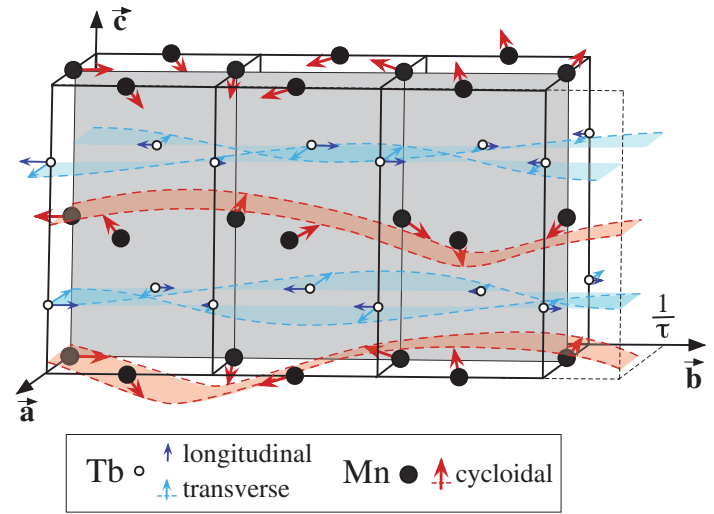

Figure 16. a) Experimental setup used for the experiment on $\mathrm{TbMnO}_{3}$ (same as Fig. 14). b) Polarization analysis of the magnetic satellites $(4, \pm \tau, \pm 1$ ) for CR (green circles), CL (red dots) and $\pi$ (black dots) incident polarizations, enregistered in the $\pi^{\prime}$ channel $\left(\eta=90^{\circ}\right)$. c) Full polarisation analysis of the diffracted intensities of the $(4,-\tau,-1)$ magnetic reflection taken in the incommensurate ferroelectric phase at $\mathrm{T}=14 \mathrm{~K}$ as a function of the electric field annealing $(\mathrm{E} \pm 700 \mathrm{~V} / \mathrm{mm})$. The dotted lines correspond to the model deduced from neutron scattering [90], where only the sinusoidal transverse component of $\mathrm{Tb}$ magnetic moment is taken into account. The continuous lines are obtained by considering also the longitudinal sinusoidal contribution of $\mathrm{Tb}$ moments. d) Magnetic structure of $\mathrm{TbMnO}_{3}$ determined by the polarization analysis of four magnetic satellite reflections $(4, \pm \tau, \pm 1)$. The Mn moments (red arrows) have a cycloidal magnetic structure with the same incommensurate periodicity $1 / \tau$ as the sinusoidal transverse (pale blue) and longitudinal (deep blue) modulation of the Tb magnetic moments. Notice the detailed anti-phase relations between the magnetic moments of the two ordered sublattices (from Ref. [87]).

have been analyzed in detail. The resultant magnetic structure is shown in Figure 16d, in which both the $\mathrm{Tb}$ and Mn magnetic moments are represented [87].

The effect of a static magnetic field on a system of magnetically ordered atoms is, in general, to induce a uniform ferromagnetic component parallel to the applied magnetic field. In non-collinear magnetic systems like $\mathrm{TbMnO}_{3}$, the application of a sufficiently strong magnetic field can rotate the plane of the cycloid [91] and hence rotate the direction of the measured electric polarization defined by the antisymmetric Dzyaloshinskii-Moriya coupling (see Fig. 13). Here we are particularly interested in the symmetry breaking effect of a magnetic field applied in the ferroic phase, in order to study the spin-lattice coupling. A non-resonant X-ray scattering investigation of $\mathrm{TbMnO}_{3}$ under applied magnetic field has revealed the possibility of finding magnetic and charge displacive amplitudes of the same order of magnitude superposed at the same reflection. By analyzing the interference between the magnetic and charge displacive amplitudes, we are able to obtain detailed information regarding the nature of the displacements as a function of applied magnetic field. This interference method is explained in the example of Figure 5, and is very sensitive to displacements on the femtometer scale: in effect the 


\section{Collection SFN}

a)

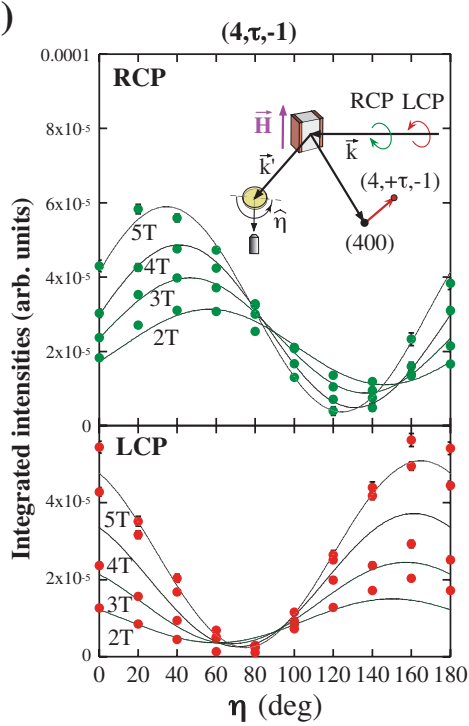

b)

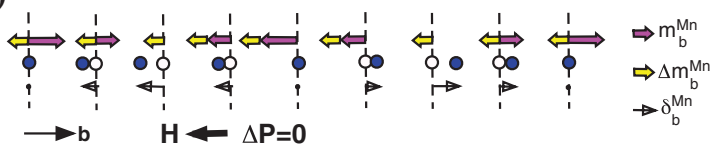

c)
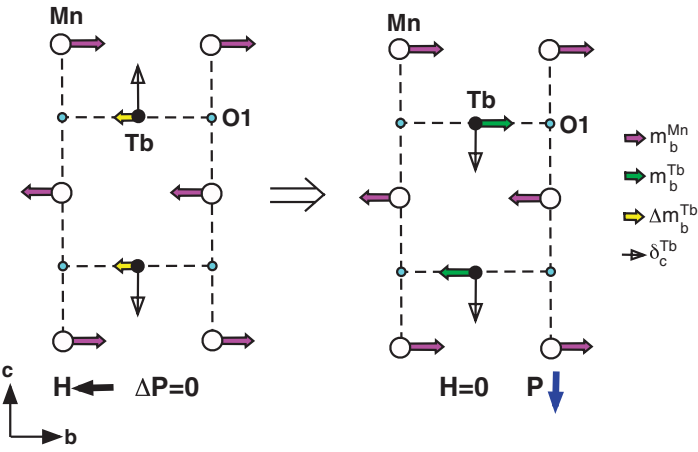

Figure 17. a) Polarisation analysis of the magnetic reflection $(4, \tau,-1)$ as a function of the magnetic field $\mathrm{H}$ applied along the $\mathbf{b}$ direction in $\mathrm{TbMnO}_{3}$. The continuous lines are calculated with an interference model, which takes into account the magnetic and Thomson scattering amplitudes. b) The application of the magnetic field along the $\mathbf{b}$ axis of the crystal induces a uniform ferromagnetic component $\Delta \mathrm{m}_{b}^{M n}$ (yellow arrows) which sums with the Mn magnetic moment $\mathrm{m}_{b}^{M n}$ (pink arrows) to produce a variation in magnetisation density between nearest neighbours. This establishes atomic displacements $\delta_{b}^{b M n}$ with the same periodicity as the magnetic cycloid. c) (left panel) Due to the symmetric exchange striction interaction between magnetic field induced $\Delta \mathrm{m}_{b}^{T b}$ and $\mathrm{m}_{b}^{M n}$, an antiphase displacement $\delta_{c}^{T b}$ is established along the c axis, resulting in zero change in the $\mathbf{P}$ (right panel). Knowing how the displacements vary as a function of field, it is possible to estimate the displacements in zero field, and therefore to determine the contribution of this symmetric exchange effect to the overall electric polarization. (From Ref. [89].)

Thomson amplitude interferes with the magnetic amplitude, known by its structure factor, and therefore it is determined in absolute units with its correct phase.

As shown in Figure 17a, when the magnetic field $\mathrm{H}$ was applied along the b-axis of the sample, on analysing the XR polarization of the magnetic scattered intensities as a function of the applied magnetic field, the signal produced by the atomic displacements interferes with the magnetic scattering amplitude. Indeed, the polarisation analysis of the magnetic reflection $(4, \tau,-1)$ as a function of the magnetic field $\mathbf{H}$ applied along the b direction shows an extra contribution which increases with $H$, and is superposed to the magnetic signal. This contribution is attributed to the charge displacement wave, due to a physical mechanism associated with the symmetric exchange striction that is produced on creating an imbalance in the magnetization density between nearest neighbors. There are no reports in the literature of a change in the magnetic structure below the spin flop transition field (flop of the cycloidal plane), and indeed these data could only be fitted by including charge Thomson scattering into our calculations for the scattering cross-section, as described in Eq. (3.16).

The physical origin of the charge scattering is the magnetostrictive atomic displacements due to interactions between the zero field moments and those induced by the applied magnetic field, which have the same periodicity as the magnetic ordering (Fig. 17b). The analysis of this interference phenomenon for several magnetic reflections, taking the group symmetry compatible with the applied magnetic field into account, allowed us to extract a detailed model for the induced ionic displacements. One of the physical mechanisms responsible for these displacements is associated with the symmetric exchange striction that is produced on creating an imbalance in the magnetization density between 
nearest neighbours as described in Figure 17b,c. The absolute measurement of the ionic displacements as a function of the magnetic field leads us to draw conclusions concerning the microscopic coupling mechanism in the ferroic phase in zero magnetic field, such as the necessity to include certain symmetric exchange contributions to the bulk electric polarization, and to estimate the magnitude of the induced polarization [89].

A specific feature of these experiments is that they were performed at three different energies to enable the separation of the signals arising from the $\mathrm{Tb}$ and $\mathrm{Mn}$ ions through the changing ratio of the dispersion corrections for the atomic scattering factors. By analysing the results for two reflections $(4, \tau, \pm 1)$ at the three energies we have established different displacement modes [92]. One of the dominant modes responsible for the charge scattering is the Tb motion of $192 \mathrm{fm} / \mathrm{T}$ along the c-axis. It is the interaction between the $\mathrm{Tb}$ induced moment along $\mathrm{b}$ with the zero field parallel component of the $\mathrm{Mn}$ moment which leads to this displacement. These displacements are in anti-phase and therefore sum to give zero net electric polarisation. By relating the size of the induced moment along $\mathrm{b}$ on the $\mathrm{Tb}$ ions in a magnetic field, with the magnitude of the b-component of the moment in zero field, we can estimate the magnitude of the zero field atomic displacements, obtaining $21 \pm 3 \mathrm{fm}$ for the Tb displacements along c. However, in contrast to the magnetic field situation, in zero field these displacements are in phase along c (Fig. 1c), giving an electric polarisation of $150 \mu \mathrm{C} / \mathrm{m}^{2}$. Thus we have revealed a mechanism concurrent to the Dzyaloshinskii-Moriya interaction, due to a symmetric interaction between parallel moments, which produces an electric polarisation which accounts for one quarter of that observed in bulk measurements.

This new XR experimental technique uses the magnetic field to control and tune the effects of magnetostriction in complex non-ferromagnetic systems, and permits the study of spin-lattice coupling terms with unprecedented accuracy.

\section{SUMMARY AND CONCLUSIONS}

The combination of $\mathrm{x}$-ray atomic spectroscopy and diffraction at synchrotron radiation facilities offers a new perspective in the investigation of electronic and magnetic ordering phenomena in condensed matter physics. The main advantage with respect to the absorption spectroscopic methods is the possibility to coherently disentangle the electric and the magnetic degree of freedom by using the combined spectroscopic, structural and crystallographic methods. This is particularly suitable in the study of strongly correlated electron systems, in which the complex interaction between the charge, orbital and spin degree of freedom can determine peculiar phenomena like superconductivity, colossal magnetoresistance, metal-insulator transition, charge ordering and orbital ordering.

This manuscript summarizes the experimental and the theoretical achievements in the domain of magnetic and resonant x-ray scattering, describing a set of experiments done during the last ten years and which present an increasing complexity in term of experimental and physical interpretation of the scattering phenomena. Indeed, during this last decade we have demonstrated the potential of these methods by improving the experimental setup in synergy with the theoretical analysis and interpretation of the experimental data, pushing the evolution of the x-ray instrumentation to the limit of the measurability, and exploiting all the main characteristic of third generation synchrotron radiation sources: high brillance, high polarization rate, high Q-resolution.

The main scientific case for RXS in the hard x-ray regime is focused on measurements of the diffracted intensity of forbidden lattice reflections appearing as a consequence of broken lattice symmetries (magnetic, charge or multipolar), and characterizing them in terms of light polarization, angular dependence about the scattering vector (azimuthal dependence), and energy dependence across the absorption edge. The shell and the chemical selectivity of the RXS technique allows to probe directly the $3 \mathrm{~d}$ and the $4 \mathrm{p}$ shells of transition metals (K-edge), the $4 \mathrm{f}$ or the $5 \mathrm{~d}$ shells of lanthanides (L-edges), and the $6 \mathrm{~d}$ or the $5 \mathrm{f}$ shells of actinides (L- and M-edges). The azimuthal dependence is a method which 


\section{Collection SFN}

directly probes the symmetries of the tensors involved in resonant scattering amplitudes by measuring the diffracted x-ray linear polarization upon rotating the sample around the scattering vector.

The possibility to control the incident beam polarization by diamond phase plates and to analyze the polarization of the scattered photons as a function of the azimuthal scattering geometry and the incident energy will play an important role in the future development of synchrotron based methods, providing a way to obtain information on the symmetry and the nature of resonances when several multipole excitations close in energy contribute to the same transition.

This technique has been essential in a number of high-profile cases, as for example the orbital and charge ordering in transition metals [94-101], frustrated magnetism in low dimensional magnetic systems [102, 103], multiferroics [104, 105], heavy fermion antiferromagnets [106, 107], picnide superconductors [108-110], magnetic thin films and multilayers [111, 112].

High order multipoles have been detected in actinide compounds [113-117], rare earth compounds [118] and transition metal compounds [76, 119, 120]. The knowledge of the electric and magnetic multipoles of both parities under space-inversion and time-reversal can be of great importance in order to understand the physics of strongly correlated electron systems, and their investigation has been a common theme of research during the last 20 years. In the light of this interpretation, the RXS technique can be applied to the investigation of many complex materials, such as Mott insulators, colossal magnetoresistive materials, actinides, high-Tc superconductors, multiferroics, heavy fermions and other transition metal oxides, in which orbital or multi-polar hidden order parameters coexist and influence the phase transitions. The separation of the resonant signal in terms of electromagnetic multipoles is, at present, one of the most fashionable ways to classify these experiments and opens new avenues in this research field.

In the non-resonant magnetic scattering regime, the studies of multiferroics has shown the importance of circular polarization in the determination of the non-collinear and incommensurate magnetic structures, a powerful method which could complement neutron scattering in the determination of complex phase relationship between different magnetic sublattices. Notice that by using the circular polarization the absolute sign of the Dzyaloshinskii-Moriya interaction can be known. Indeed, the possibility to control the helicity of the incident polarization with a polarization rate close to $99 \%$ and to analyse the scattered photon polarization is essential for the determination of the magnetic domain population in chiral systems, and in favorable cases also the absolute phase relationships between different magnetic sublattices. The improvement of $\mathrm{X}$-ray focusing and the high-Q resolution properties of the X-rays could be essential in future developments of this technique to study and to image the magnetic domain evolution under combined external extreme conditions like high magnetic and electric fields, and potentially also high pressure studies.

It is also important to highlight the complementarities of the hard RXS methods with respect to the soft RXS and the absorption spectroscopies, like circular and linear dichroism. The hard x-ray regime, despite the weakness of resonances (for example at K-edges of transition metals), could give access to the investigation of resonant phenomena in different Brillouin zones, exploiting the atomic site selectivity by the structure factor selection rules, a unique way to analyze complex magnetic or electronic long range structure and their evolution through the phase transitions.

\section{Acknowledgements}

The main acknowledgement is addressed to all the staff of the past ID20 beam line at ESRF, which have work during the last ten years to develop and optimise the instrumentation of this unique and highly specialised beam line, now dismounted. This manuscript summarise the main experimental and theoretical achievements of students, post-docs, scientists and collaborators which have contributed at different levels to the experiments reported here. 


\section{References}

[1] Klein, O. \& Nishina, Y., Zeit. Phys. 52, (1929) 853-868; Nishina, Y., ibid. 869-877.

[2] Compton, A.H., Physical Review 50, (1936) 878-881.

[3] Gell-Mann, M. \& Goldberger, M.J., Physical Review 96, (1954) 1433-1438.

[4] Low, F.E., Physical Review 96, (1954)1428-1432.

[5] Tolhoek, H.A., Rev. Mod. Phys. 28, (1956) 277-298.

[6] de Bergevin, F. \& Brunel, M., Phys. Letters A39, (1972) 141-142.

[7] de Bergevin, F. \& Brunel, M., Acta Cryst. A37, (1981) 314- 331; ibid. 324-331.

[8] Platzman, P.M. \& Tzoar, N., Physical Review B 2, (1970) 3556-3559.

[9] Blume, M., J. Appl. Phys. 57, (1985) 3615-3618.

[10] Blume, M., Resonant Anomalous X-ray scattering, edited by G. Materlik, J. Sparks \& K. Fisher, (1994) p. 495-512. Elsevier Science B.V., Amsterdam.

[11] Blume, M. and Gibbs, D., Physical Review B 37, (1988) 1779-1789.

[12] Altarelli, M., Resonant X-ray scattering: a theoretical introduction. Lecture Notes in Physics, (Springer 2006), vol. 697, p. 201.

[13] Hannon, J.P., Trammel, G.T., Blume, M. \& Gibbs, D., Physical Review Letters 61, (1988) 12451248.

[14] Hill, J. and McMorrow, D., Acta Cryst. A 52 (1996) 236-244.

[15] Paolasini, L., Vettier, C., de Bergevin, F., Yakhou, F., Mannix, D., Stunault, A., Neubeck, W., Altarelli, M., Fabrizio M., Metcalf P.A., Honig J.M., Orbital Occupancy Order in $\mathrm{V}_{2} \mathrm{O}_{3}$ : Resonant X-Ray Scattering Results. Physical Review Letters 82 (1999) 4719-4722.

[16] Marri, I. \& Carra P., Physical Review B 69, (2004) 113101(1-4).

[17] Loversey, S., Balcar, E., Knight, K.S., Fernández-Rodríguez, J. Physics Reports 411, (2005) 233-289.

[18] Di Matteo, S. Resonant X-ray Diffraction: Multipole interpretation, J. Phys. D: Appl. Phys. 45 (2012) 163001.

[19] Fano, U. and Rau, A.R.P., in: "Symmetry in quantum physics" Academic press (1996), ISBN 0-12-248455. Physics Reports 187 (1990) 145-202.

[20] Paolasini, L. and de Bergevin, F., "Magnetic and resonant X-ray scattering investigations of strongly correlated electron systems" C.R. Physique 9 (2008) 550-569.

[21] Di Matteo, S., Joly, Y., Natoli, C.R., Detection of electromagnetic multipoles by $x$-ray spectroscopies. Physical Review B 72 (2005)144406,1-6.

[22] Di Matteo, S.J. Phys. Conf. Series 211 (2010) 012008.

[23] Joly, Y., Physical Review B 63 (2001) 125120(1-11).

[24] Joly, Y., Di Matteo, S. \& Natoli, C.R., Physical Review B 69, (2004) 224401(1-11).

[25] Lovesey, S.W. and Balcar, E., J. Phys. Soc. Japan 79 (2010) 104702.

[26] Templeton, L.K., Templeton, D.H., Phizackerley, R.P, Hodgson, K.O., Acta Cryst. A38, (1982) 74-78.

[27] Dmitrienko, V.E., Acta Cryst. A 39, (1983) 29-35.

[28] Finkelstein, K.D., Shen, Q., Shastri, S., Physical Review Letters 69 (1992) 1612-1615.

[29] Carra P., Thole B.T., Rev. Mod. Phys. 66 (1994) 1509-1515.

[30] Thole, B.T., Carra, P., Sette, F., and van der Laan, G., Physical Review Letters 68 (1992) 1943.

[31] Di Matteo, S. and Natoli, C.R., J. Synchrotron Radiat. 9, (2002) 9.

[32] Vettier, C., J. Elect. Spectr. and Rel. Phen. 117-118, (2001) 113-128.

[33] Detlefs, C., A.H.M.Z. Islam, A.I. Goldman, C. Stassis, P. Canfield, J.P. Hill, D. Gibbs, Phys. Rev. B 55, (1997) R680-683.

[34] Paolasini, L., Detlefs, C., Mazzoli, C., Wilkins, S., Deen, P., Bombardi, A., Kernavanois, N., de Bergevin, F., Yakhou, F., Valade, J.P., Breslavetz, I., Fondacaro, A., Pepellin, G., Bernard, 


\section{Collection SFN}

P., ID20: a beamline for magnetic and resonant $x$-ray scattering investigations under extreme conditions. J. Synchrotron Rad. 14 (2007) 301-312.

[35] Paolasini, L. and de Bergevin, F., Magnetic and resonant x-ray scattering investigations of strongly correlated electron systems. Compte Rendus Physique 9, (2008) 550-569.

[36] Gibbs, D., Blume, M. Harshman, D.R., McWhan, D.B., Rev. Sci. Instrum 60 (1989) 1655.

[37] Fernandez, V. Vettier, C., de Bergevin, F., Giles, C., Neubeck, W., Physical Review B 57, (1998) 7870-7876.

[38] Neubeck, W., Vettier, C., de Bergevin, F., Yakhou, F., Mannix, D., Ranno, L., Chatterji, T., J. of Phys. and Chem. of Solids 62, (2001) 2173-2180.

[39] Langridge, S.L., Lander, G.H., Bernhoeft, N., Stunault, A., Vettier, C., Grübel, G., Sutter, C., de Bergevin, F., Nuttall, W.J., Stirling, W.G., Mattenberger, K., Vogt, O., Physical Review B 55, (1997) 6392-6398.

[40] Caciuffo, R., Paolasini, L., Sollier, A., Ghigna, P., Pavarini, E., van der Brink, J. and Altarelli, M., Resonant $x$-ray scattering study of magnetic and orbital order in $\mathrm{KCu}_{3}$. Physical Review B 65, (2002) 174425(1-9).

[41] Skalicky, P. and Malgrange, C., Acta Cryst. A 28 (1972) 501.

[42] Hirano, K., Kanzaki, K., Mikami, M., Miura, M., Tamasaku, K., Ishikawa, T., \& Kikuta S., J. Appl. Cryst. 25 (1992) 531-535.

[43] Giles, C., Malgrange, C., Goulon, J., de Bergevin, F., Vettier, C., Dartyge, E., Fontaine, A., Giogetti, C. \& Piz zini S., J. Appl. Cryst. 27 (1994) 27, 232-240.

[44] Giles, C., Malgrange, C., Goulon, J., de Bergevin, F., Vettier, C., Fontaine, A., Dartyge, E., Pizzini, S., Baudelet, F. \& Freund., A., Rev. Sci. Instrum. 66 (1995) 1549-1553.

[45] Giles, C., C. Vettier, C., Ê de Bergevin, F., Malgrange, C., Grübel, G., \& Grossi, F., Rev. Sci. Instrum. 66 (1995) 1518-1521.

[46] Okitsu, K., Ueji, Y., Sato, K., \& Amemiya, Y., J. Synchrotron Rad. 8 (2001) 33-37.

[47] Fano U., Rev. Mod. Phys. 29 (1957) 74.

[48] Scagnoli, V., Mazzoli, C., Detlefs, C., Bernard, P., Fondacaro, A., Paolasini, L., Fabrizi, F., de Bergevin, F., Linear polarization scans for resonant X-ray diffraction with a double-phase-plate configuration, Journal of Synchrotron Radiation 16, 778-787 (2009).

[49] d'Astuto, M., Barla, A., Kernavanois, N., Rueff, J-P, Baudelet, F., Rüffer, R., Paolasini, L. \& Couzinet, B., Lecture Notes in Physics 697 (2006) 375-399.

[50] Kernavanois, N., Deen, P., Braithwaite, D. and Paolasini, L., Pressure device for resonant magnetic $x$-ray scattering . Rev. Scient. Instr. 76, (2005) 083909(1-5).

[51] Braithwaite, D., Paolasini, L., Deen, P.P., Kernavanois, N., Yakhou, F., Canfield, P., Lapertot, G. High-pressure resonant magnetic $\mathrm{X}$-ray diffraction and transport experiments in $\mathrm{Ce}\left(\mathrm{Fe}_{1-x} \mathrm{Co}_{x}\right)_{2}$, Physica B 378-380, 782-783 (2006).

[52] Deen, P.P., Braithwaite, D., Kernavanois, N., Paolasini, L., Raymond, S., Barla, A., Lapertot, G., Sanchez, J-P, Structural and electronic transitions in the low-temperature, high-pressure phase of SmS. Physical Review B 71, (2005) 245118(1-5).

[53] Goodenough, J.B., Magnetism and chemical Bond, Interscience, New York, 1963.

[54] Kugel', K.I. and Khomskii, D.I., Zh. Eksp. Teor. Fiz. 64, (1973) 1429; [Sov. Phys. JEPT 37 (1973) 725].

[55] Kugel', K.I. and Khomskii, D.I., Usp. Fiz. Nauk 136, (1982) 1982; [Sov. Phys. Usp. 25 (1982) 231].

[56] Hutchings, M.T, Samuelsen, E.J., Shirane, G. \& Hirakawa, K., Physical Review 188, (1969) 919-923.

[57] Tennant, D.A., Perring, T.G., Cowley, R.A., Nagler, S.E., Physical Review Letters 70 (1993) 4003.

[58] Lake, B., Tennant, D.A., Nagler, S.E., Physical Review Letters 85 (2000) 832. 
[59] Murakami, Y., Kawada, H., Kawata, H., Tanaka, M., Arima, T., Moritomo, Y., Tokura, Y., Physical Review Letters 80 (1998) 1932-1935.

[60] Paolasini, L., Caciuffo, R., Sollier, A., Ghigna, P., and Altarelli, M., Coupling between Spin and Orbital Degrees of Freedom in $\mathrm{KCuF}_{3}$. Physical Review Letters 88 (2002) 106403.

[61] Deisenhofer, J., Leonov, I., Eremin, M.V., Kant, Ch., Ghigna, P., Mayr, F., Iglamov, V.V., Anisimov, V.I., and van der Marel, D., Physical Review Letters 101 (2008) 157406.

[62] Mazzoli, C., Paolasini, L., de Bergevin, F., Ghigna, P., Caciuffo, R., New findings in Resonant $X$-ray Scattering investigation of $\mathrm{KCu}_{3}$ orbital model system, Physica B 378-380 (2006) 563.

[63] Mott N.F., Metal insulator transitions, Taylor and Francis, London 1974.

[64] Moon, R.M., Physical Review Letters 25, (1970) 527-529.

[65] McWhan, D.B. and Remeika, J.P., Physical Review B 2 (1970) 3734.

[66] Castellani, C., Natoli, C.R., Ranninger, J., Physical Review B 18 (1978) 4967.

[67] Fabrizio, M., Altarelli, M. and Benfatto, M., Physical Review Letters 80 (1998) 3400.

[68] Park, J-H, Tjeng, L.H., Tanaka, A., Allen, J.W., Chen, C.T., Metcalf, P., Honig, J.M., de Groot, F., Sawatzky, G.A., Phys. Rev. B 61 (2000) 11506.

[69] Mila, F., Shiina, R., Zhang, F-C, Joshi, A., Ma, M., Anisimov, V. and Rice, T.M., Physical Review Letters 85(2000) 1714.

[70] Di Matteo, S., Perkins, N.B. and Natoli, C.R., Physical Review B 65 (2002) 054413.

[71] Tanaka, A., J. Phys. Soc. Japan 71 (2002) 1091.

[72] Paolasini, L., Di Matteo, S., Vettier, C., de Bergevin, F., Sollier, A., Neubeck, W., Yakhou, F., Metcalf, P.A., Honig, J.M., Interplay between orbital and magnetic long range order by resonant $X$-ray scattering in $\left(\mathrm{V}_{1-x} \mathrm{Cr}_{x}\right)_{2} \mathrm{O}_{3}$, J. Elect. Spectr. and Rel. Phen. 120, (2001) 1-10.

[73] Lovesey, S.W., Knight, K.S. and Sivia, D.S., Physical Review B 65 (2002) 224402.

[74] Di Matteo, S., Joly, Y., Bombardi, A., Paolasini, L., de Bergevin F. \& Natoli, C.R., Local ChiralSymmetry Breaking in Globally Centrosymmetric Crystals. Physical Review Letters 91 (2003) 257402(1-4).

[75] Bombardi, A., de Bergevin, F., Di Matteo, S., Paolasini, L., Precursor symmetry breaking in Cr doped $\mathrm{V}_{2} \mathrm{O}_{3}$. Physica B 345 (2004) 40-44.

[76] Mazzoli, C., Wilkins, S.B., Di Matteo, S., Detlefs, B., Detlefs, C., Scagnoli, V., Paolasini, L., Ghigna, P., - Disentangling multipole resonances through a full X-ray polarization analysis, Physical Review B 76, 195118-1-195118-6 (2007).

[77] Loversey, S., Fernandez-Rodriguez, J., Blanco, J.A., Sivia, D.S., Knight, K.S., Paolasini, L., Vanadium magnetoelectric multipoles in $\mathrm{V}_{2} \mathrm{O}_{3}$, Physical Review B 75014409 (2007).

[78] Fernández-Rodríguez, J., Scagnoli, V., Mazzoli, C., Fabrizi, F., Lovesey, S.W., Blanco, J.A., Sivia, D.S., Knight, K.S., de Bergevin, F., Paolasini, L., Experimental evidence of anapolar moments in the antiferromagnetic insulating phase of $\mathrm{V}_{2} \mathrm{O}_{3}$ obtained from x-ray resonant Bragg diffraction, Physical Review B 81, 085107-1-085107-7 (2010).

[79] Kimura, T., Goto, T., Shintani, H., Ishizaka, K., Arima, T., Tokura, Y., Nature 426, 55 (2003).

[80] Katsura, H., Nagaosa, N., Balatsky, A.V., Physical Review Letters 95 (2005) 057205.

[81] Mostovoy, M., Physical Review Letters 96 (2006) 067601.

[82] Fabrizi, F., Walker, H.C., Paolasini, L., de Bergevin, F., Fennel, T., Rogado, N., Cava, R.J., Wolf, Th. Kenzelmann, M., McMorrow, D.F., Electric field control of multiferroic domains in $\mathrm{Ni}_{3} \mathrm{~V}_{2} \mathrm{O}_{8}$ imaged by $\mathrm{X}$-ray polarization enhanced topography, Physical Review B 82, 024434 (2010).

[83] Fabrizi, F.: "Probing magnetism in magneto-electric multiferroics using circularly polarized $X$-rays". PhD thesis at University College London, UK, Feb. 2011.

[84] Kenzelmann, M., Harris, A.B., Aharony, A., Entin-Wohlman, O., Yildirim, T., Huang, Q., Park, S., Lawes, G., Broholm, C., Rogado, N., Cava, R.J., Kim, K.H., Jorge, G., and Ramirez, A.P., Physical Review B 74 (2006) 014429. 


\section{Collection SFN}

[85] Lawes, G., Harris, A.B., Kimura, T., Rogado, N., Cava, R.J., Aharony, A., Entin-Wohlman, O., Yildirim, T., Kenzelmann, M., Broholm, C. and Ramirez, A.P., Physical Review Letters 95 (2005) 087205.

[86] Catalan, G., Seidel, J., Ramesh, R., Scott, J.F., Rev. Mod. Phys. 84 (2012) 119.

[87] Fabrizi, F., Walker, H.C., Paolasini, L., de Bergevin, F., Boothroyd, A.T., Prabhakaran, D., McMorrow, D. F.,- Circularly polarized X-rays as a probe of noncollinear magnetic order in multiferroic TbMnO 3 , Physical Review Letters 102, 237205-1-237205-4 (2009).

[88] Mannix, D., McMorrow, D.F., Ewings, R.A., Boothroyd, A.T., Prabhakaran, D., Joly, Y., Janousová, B., Mazzoli, C., Paolasini, L., Wilkins, S.B., X-ray scattering study of the order parameters in multiferroic TbMnO $\mathrm{T}_{3}$, Physical Review B 76 (2007) 184420-1-184420-17.

[89] Walker, H.C., Fabrizi, F., Paolasini, L., de Bergevin, F., Herrero-Martin, J., Boothroyd, A.T., Prabhakaran, D., McMorrow, D.F., Femtoscale magnetically induced lattice distortion in multiferroic $\mathrm{TbMnO}_{3}$, Science 333 (2011) 1273.

[90] Kenzelmann, M., Harris, A.B., Jonas, S., Broholm, C., Schefer, J., Kim, S.B., Zhang, C.L., Cheong, S.-W., Vajk, O.P., and Lynn, J.W., Physical Review Letters 95, (2005) 087206.

[91] Aliouane, N., Schmalzl, K., Senff, D., Maljuk, A., Prokes, K., Braden, M., and Argyriou, D.N., Physical Review Letters 102 (2009) 207205.

[92] Walker, H.C., Fabrizi, F., Paolasini, L., de Bergevin, F., Prabhakaran, D., Boothroyd, A.T., McMorrow, D.F., Circularly polarized x-ray scattering investigation of spin-lattice coupling in $\mathrm{TbMnO}_{3}$ in crossed electric and magnetic fields, Physical Review B 88 (2013) 214415-1214415-13.

[93] Staub, U., Meijer, G.I., Fauth, F., Allenspach, R., Bednorz, G., Karpinski, J., Kazakov, S., Paolasini, L. and dÕAcapito, F., Charge driven metal-insulator transition in $\mathrm{NdNiO}_{3}$, Physical Review Letters 88 (2002) 126402.

[94] Fagot, S., Foury-Leylekian, P., Ravy, S., Pouget, J.P., Lorenzo, E., Joly, Y., Greenblatt, M., Lobanov, M.V., Popov, G., X-ray anomalous scattering investigation of BaV $S_{3}$, Physical Review B 73, 033102-1-033102-4 (2006).

[95] Lorenzo, J.E., Mazzoli, C., Jaouen, N., Detlefs, C., Mannix, D., Grenier, S., Joly, Y., Marin, C., Charge and orbital correlations at and above the Verwey phase transition in magnetite, Physical Review Letters 101, 226401-1-226401-4 (2008).

[96] García-Fernández, M., Scagnoli, V., Staub, U., Mulders, A.M., Janousch, M., Bodenthin, Y., Meister, D., Patterson, B.D., Mirone, A., Tanaka, Y., Nakamura, T., Grenier, S., Huang, Y., Conder, K., Magnetic and electronic Co states in the layered cobaltate $\mathrm{GdBaCo}_{2} \mathrm{O}_{5.5-x}$, Physical Review B 78 (2008) 054424.

[97] Bland, S.R., Detlefs, B., Wilkins, S.B., Beale, T.A.W., Mazzoli, C., Joly, Y., Hatton, P.D., Lorenzo, J.E., and Brabers, V.A.M., Full Polarization Analysis of Resonant Superlattice and Forbidden X-ray ReSSections in Magnetite, J. Phys. Condens Matt. 21, (2009) 485601.

[98] Foury-Leylekian, P., Poltavets, V.V., Jaouen, N., Rueff, J.P., Lorenzo, J.E., Auban-Senzier, P., Pasquier, C.R., Mazzoli, C., Greenblatt, M., Sodium ion and cobalt charge ordering in $\mathrm{Na}_{x} \mathrm{CoO}_{2}\left(x \approx \frac{5}{6}\right)$, Physical Review B 79 (2009) 115101.

[99] García, J., Subías, G., Herrero-Martín, J., Blasco, J., Cuartero, V., Sánchez, M.C., Mazzoli, C., Yakhou F., Reexamination of the temperature dependence of resonant reflections in highly stoichiometric magnetite, Physical Review Letters 102, 176405-1-176405-4 (2009).

[100] Mulders, A.M., Lawrence, S.M., Staub, U., García-Fernández, M., Scagnoli, V., Mazzoli, C., Pomjakushina, E., Conder, K., Wang, Y., Direct observation of charge order and an orbital glass state in multiferroic $\mathrm{LuFe}_{2} \mathrm{O}_{4}$, Physical Review Letters 103 (2009) 077602.

[101] Bland, S.R., Angst, M., Adiga, S., Scagnoli, V., Johnson, R.D., Herrero-Martin, J., and Hatton, P., A study of symmetry and charge order in $\mathrm{Fe}_{2} \mathrm{OBO}_{3}$ through polarized resonant $x$-ray diffraction, Physical Review B, 82, 115110 (2010). 
[102] Bombardi A., Rodriguez-Carvajal J., Di Matteo S., De Bergevin F., Paolasini L., Carretta P., Millet P. and Caciuffo R., Direct determination of the magnetic ground state in the square lattice $\mathrm{S}=1 / 2$ antiferromagnet $\mathrm{Li}_{2} \mathrm{~V} \mathrm{OSi}_{4}$, Physical Review Letters 93 (2004) 027202.

[103] Agrestini S., Mazzoli C., Bombardi A., Lees M.R., Incommensurate magnetic ground state revealed by resonant $X$-ray scattering in the frustrated spin system $\mathrm{Ca}_{3} \mathrm{Co}_{2} \mathrm{O}_{6}$, Physical Review B 77, 140403-1-140403-4 (2008).

[104] Mannix D., McMorrow D.F., Ewings R.A., Boothroyd A.T., Prabhakaran D., Joly Y., Janousova B., Mazzoli C., Paolasini L., Wilkins S.B., X-ray Resonant Scattering Study of Order Parameters in Multiferroic $\mathrm{TbMnO}_{3}$, Physical Review B 76 (2007) 184420.

[105] Johnson R.D., Bland S.R., Mazzoli C., Beale T.A.W., Du C.H., Detlefs C., Wilkins S.B., Hatton P.D., Determination of magnetic order of the rare earth ions in multiferroic $\mathrm{TbMn}_{2} \mathrm{O}_{5}$, Physical Review B 78, 104407-1-104407-9 (2008).

[106] Adriano C., Lora-Serrano R., Giles C., de Bergevin F., Lang J.C., Srajer G., Mazzoli C., Paolasini L. and Pagliuso P.G., Magnetic structure of $\mathrm{Sm}_{2} \mathrm{Ir}_{\mathrm{In}}$ by $x$-ray resonant magnetic scattering, Physical Review B 76 (2007) 104515.

[107] Adriano C., Giles C., Bittar E.M., Coelho L.N., de Bergevin F., Mazzoli C., Paolasini L., Ratcli W., Bindel R., Lynn J.W., Fisk Z., and Pagliuso P.G., Cd-doping e? ects in $\mathrm{Ce}_{2} \mathrm{MIn}_{8}(M=R h$ and Ir) heavy fermion compounds, Physical Review B 81 (2010) 245115.

[108] Herrero-Martín J., Scagnoli V., Mazzoli C., Su Y., Mittal R., Xiao Y., Brueckel T., Kumar N., Dhar S.K., Thamizhavel A., Paolasini L., Magnetic structure of $\mathrm{EuFe}_{2} \mathrm{As}_{2}$ as determined by resonant $x$-ray scattering, Physical Review B 80, 134411-1-134411-5 (2009).

[109] Nandi, S., Su, Y., Xiao, Y., Price, S., Wang, X.F., Chen, X.H., Herrero-Martín, J., Mazzoli, C., Walker, H.C., Paolasini, L., Francoual, S., Shukla, D.K., Strempfer, J., Chatterji, T., Kumar, C. M.N., Mittal, R., Ronnow, H.M., Ruegg, Ch., McMorrow, D.F., and Bruckel, Th., Strong coupling of $\mathrm{Sm}$ and Fe magnetism in $\mathrm{SmFeAs} O$ as revealed by magnetic $x$-ray scattering, Physical Review B. 84 (2011) 054419.

[110] Herrero-Martín, J., Mazzoli, C., Scagnoli, V., Paolasini, L., Walker, H., Xiao, Y., Brueckel, T., Mittal, R., Kumar, N., Dhar, S.K., Thamizhavel, A., Su, Y., EuFe2As2: Magnetic Structure and Local Charge Distribution Anisotropies as Seen by Resonant X-ray Scattering, J. Supercond. Nov. Magn. 24 (2011) 705.

[111] Kravtsov, E., Brucas, R., Hjörvarsson, B., Hoser, A., Liebig, A., McIntyre, G.J., Miliyaev, M.A., Nefedov, A., Paolasini, L., Radu, F., Remhof, A., Ustinov, V. V., Yakhou, F., and Zabel, H., Onset of spin-density-wave antiferromagnetism in $\mathrm{Cr} / \mathrm{V}$ multilayers, Physical Review B 76 (2007) 024421.

[112] Deen, P.P., Yokaichiya, F., Paolasini, L., Lee, S., de Santis, A., Bobba, F., Cucolo, A.M., Electronic and magnetic order in $\mathrm{La}_{0.7} \mathrm{Ca}_{0.3} \mathrm{MnO}_{3} / \mathrm{YBa}_{2} \mathrm{Cu}_{3} \mathrm{O}_{7-d}$ superlattices, Journal of Magnetism and Magnetic Materials 310 (2007) 2286-2288.

[113] Wilkins, S.B., Caciuffo, R., Detlef,s C., Rebizant, J., Colineau, E., Wastin, F., Lander, G. H., Direct observation of electric-quadrupolar order in $\mathrm{UO}_{2}$, Physical Review B 73, 060406-1060406-10 (2006).

[114] McEwen, K.A., Walker, H.C., Le, M.D., McMorrow, D.F., Colineau, E., Wastin, F., Wilkins, S.B., Park, J.G., Bewley, R.I., Fort, D., Understanding the quadrupolar structures of $U P d_{3}$, Journal of Magnetism and Magnetic Materials 310, 718-722 (2007).

[115] Detlefs, B., Wilkins, S.B., Javorsky, P., Blackburn, E., Lander, G.H., Multi-k magnetic structures in $U S b_{0.9} T e_{0.1}$ and $U A s_{0.8} S e_{0.2}$ observed via resonant $X$-ray scattering at the $U M_{4}$ edge, Physical Review B 75, 174403-1-174403-6 (2007).

[116] Detlefs, B., Wilkins, S.B., Caciuffo, R., Paixão, J.A., Kaneko, K., Honda, F., Metoki, N., Bernhoeft, N., Rebizant, J., Lander, G.H., Resonant X-ray scattering study of $N p R h G a_{5}$ and NpCoGa $a_{5}$, Physical Review B 77, 024425-1-024425-10 (2008). 


\section{Collection SFN}

[117] Santini, P., Carretta, S., Amoretti, G., Caciuffo, R., Magnani, N., and Lander, G.H., Multipolar interactions in f-electron systems: The paradigm of actinide dioxide, Review of Modern Physics 81, 807- 863 (2009).

[118] Bunáu, O., Galéra, R.M., Joly, Y., Amara, M., Luca, S.E., Detlefs, C., - Resonant magnetic and multipolar scattering at the neodymium $L_{2,3}$ absorption edges in the antiferroquadrupolar phase of NdMg, Physical Review B, 81, 144402 (2010).

[119] Bombardi, A., Mazzoli, C., Agrestini, S., Lees, M.R., Resonant X-ray scattering investigation of the multipolar ordering in $\mathrm{Ca}_{3} \mathrm{Co}_{2} \mathrm{O}_{6}$, Physical Review B 78, 100406-1-100406-4 (2008).

[120] Johnson, R.D., Beale, T.A.W., Joly, Y., Bland, S.R., Hatton, P.D., Mazzoli, C., Bouchenoire, L., Prabhakaran, D. and Boothroyd, A.T., - Observed and calculated energy spectra of Braggforbidden reflections in $\mathrm{YVO}_{3}$, J. Phys. Conf. Ser., 200, 012073 (2010). 